NIST IR 7985

\title{
Towards a Classification System of Manufacturing Objects for the Evaluation of Perception Systems
}

Geraldine S. Cheok

Marek Franaszek

Afzal Godil

Kamel S. Saidi

Roger Eastman

Tsai Hong 
NIST IR 7985

\title{
Towards a Classification System of Manufacturing Objects for the Evaluation of Perception Systems
}

\author{
Geraldine S. Cheok \\ Marek Franaszek \\ Kamel S. Saidi \\ Tsai Hong \\ Intelligent Systems Division \\ Engineering Laboratory \\ Afzal Godil \\ Information Access Division \\ Information Technology Laboratory \\ Roger Eastman \\ Loyola University \\ Baltimore, $M D$
}

http://dx.doi.org/10.6028/NIST.IR.7985

February 2014

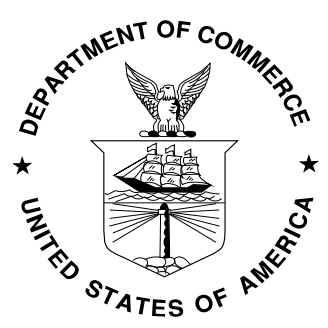

U. S. Department of Commerce Penny Pritzker, Secretary

National Institute of Standards and Technology Patrick D. Gallagher, Under Secretary of Commerce for Standards and Technology and Director 


\section{DISCLAIMER}

Certain trade names and company products are mentioned in the text or identified in an illustration in order to adequately specify the experimental procedure and equipment used. In no case does such an identification imply recommendation or endorsement by the National Institute of Standards and Technology, nor does it imply that the products are necessarily the best available for the purpose. 


\section{Contents}

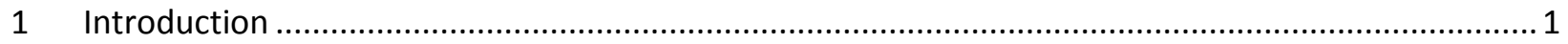

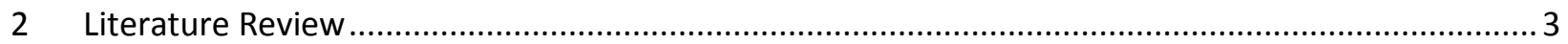

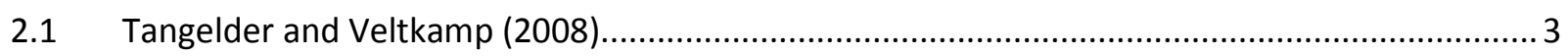

2.2 Princeton Shape Benchmark - Shillane et al. (2004) …........................................................ 4

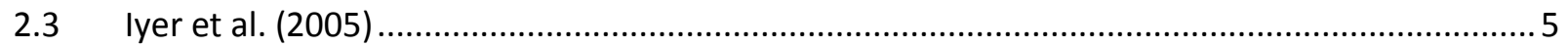

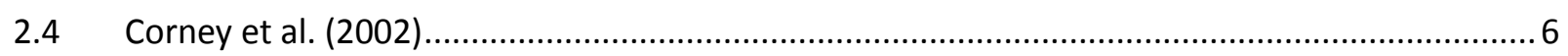

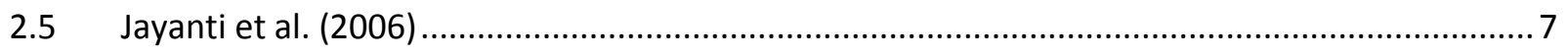

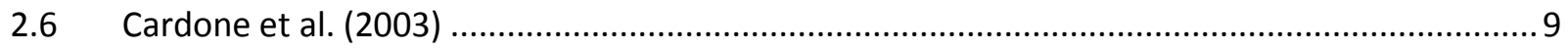

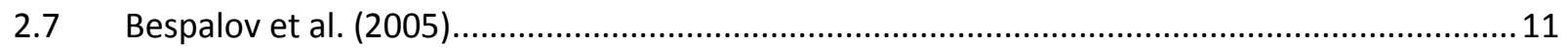

3 Object Classification for Perception Systems …................................................................... 13

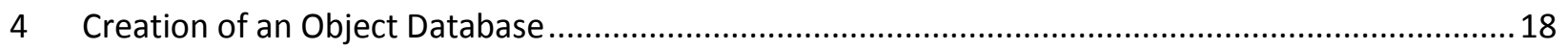

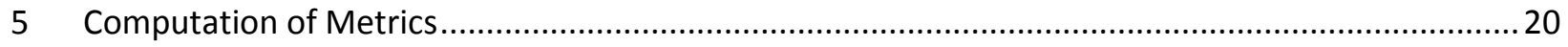

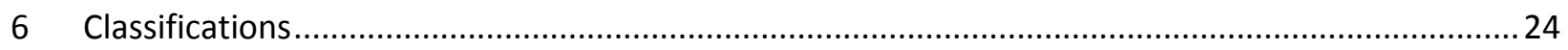

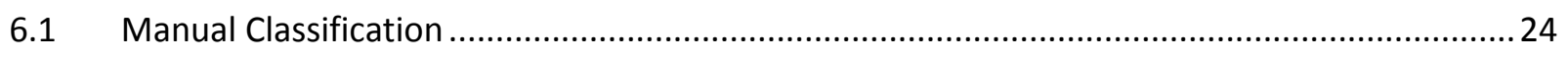

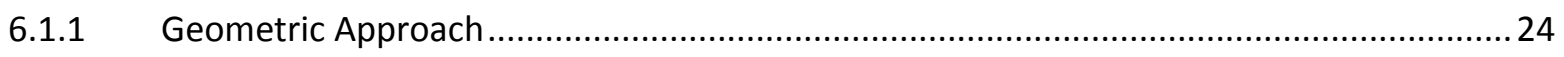

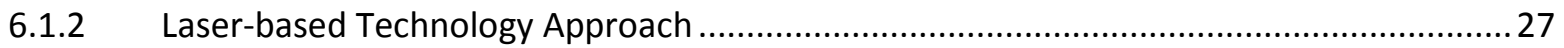

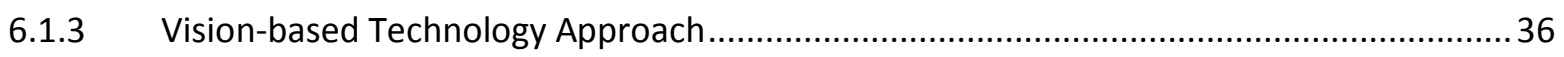

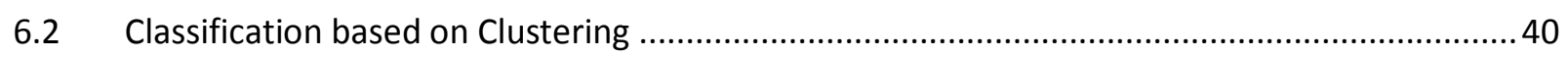

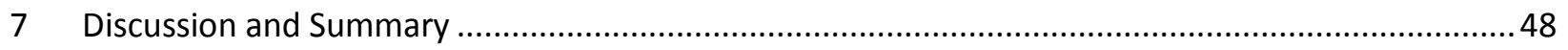

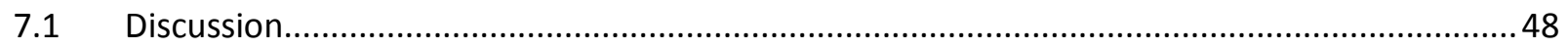

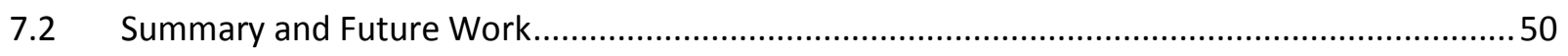

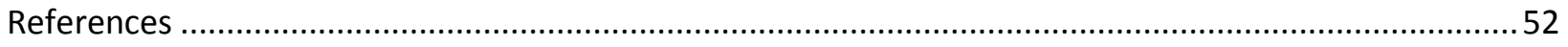

Appendix A: Objects Used in Classification Systems......................................................................... 54

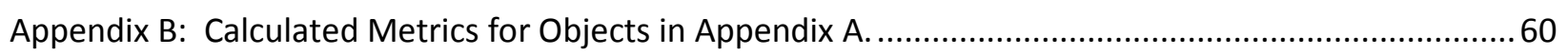

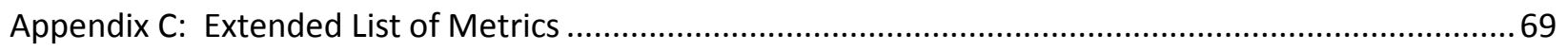




\section{Introduction}

The objective of the Robot Perception for Identifying and Locating Parts for Assembly Project is to develop test methods to evaluate perception systems used for identifying and locating unfixtured manufacturing parts under static conditions. System evaluation often involves testing the system under various conditions to determine the influence of different factors on system performance. The dimensionality of the problem is very large, including the nature of the perception system, the manufacturing application, the environmental conditions, the requirements for identification and location accuracy, and the nature of the parts. Therefore, robust test methods need to be developed that would enable evaluation for as wide a range of factors and perception systems as possible.

The evaluation of perception systems requires the use of parts or objects. However, manufacturing parts cover a wide array of parts from small parts (e.g., nuts) to large parts (e.g., airplane wings), from parts made of plastic to parts made of titanium, from parts that are flexible to parts that are rigid, and from parts that are simple (e.g., simple geometric shapes such as spheres or boxes) to parts that are very complex in shape (e.g., engine blocks). Thus, the selection of representative objects is a crucial task.

A solution to this task was to develop a part/object classification system. An object classification system will allow for the evaluation of perception systems based on objects in a particular class, and this may eventually lead to the development of standard reference artifacts of objects in the various classes.

A classification system will allow vendors to accurately specify the advantages and limitations of their systems for the different classes of objects. Such a system will also allow users to determine if a particular system would work for their specific application/object prior to making a large capital investment in the equipment, to compare various perception systems, and to have confidence in the perception system.

Manufacturing parts are naturally classified by application, material, fabrication method, function, manufacturer, and other characteristics. This study looks at commonalities in shape across natural categories, so the classification criteria may group flat parts whatever the function or fabrication method. This study focused on object shape as could be described in a computer-aided design (CAD) file, rather than taking into account surface finish for multiple reasons. Parts found under the hood of an automobile are typically kept in natural finish since their appearance is not critical. For identification, color is an obvious and powerful cue and can be easily considered after shape. The same is true of labels or heavily marked texture; and shape is key for localization and pose determination whatever the surface finish.

For the purpose of evaluating perception systems, parts can be classified by the strength of their features likely to be useful for perception, or more generally by a grading from easy to difficult to perceive. This study uses both approaches. A classification system that encompasses all manufacturing parts would be beyond the scope of this project. To keep within the project and program objectives, the scope for the classification system was narrowed down to automotive parts. Publically available CAD models were used to classify shapes manually and by numerical algorithms. A number of shape metrics were considered for classification, and a subset selected for implementation and testing. 
This report documents the efforts involved in trying to develop an object classification system for evaluating perception systems. As part of the development of an object classification system, this study reviewed previous publications that classify parts from the shape retrieval literature. This limited review is presented in Section 2 of this report. Section 3 describes some concepts that were explored or discussed when developing a classification system. Also presented in Section 3 are the metrics that can be used to characterize an object. The selection of the objects used in the classification system is presented in Section 4. Descriptions of the metrics used in this report and the how they were computed are given in Section 5. Section 6 presents the three approaches for manual classifications and an algorithmic-based approach for classification. Section 7 presents findings from the classifications and a summary. 


\section{Literature Review}

A literature review was conducted to determine existing object classification systems. The goals of many of the classification systems are for object retrieval from a database and for manufacturing systems/processes (McCarthy, 1995) (e.g., Group Technology). Classifications can be based on the part appearance and/or shape, manufacturing process to create the part, or the function of the part. There has been much research on this topic especially in the field of computer vision and pattern recognition. In the past decade, there has been an increase in interest in object retrieval in the mechanical engineering domain in part due to the widespread use of CAD and the large number of CAD models available. However, there is limited work on three-dimensional (3D) shape searching for engineering and CAD applications. The remainder of this section will summarize research and/or concepts that are relevant to $3 \mathrm{D}$ shape searching for engineering and CAD applications. Of particular interest are the metrics and methods discussed in these studies.

\subsection{Tangelder and Veltkamp (2008)}

Tangelder and Veltkamp (2008) conducted a survey of 3D shape retrieval methods. In the paper, shape matching methods are divided into: feature based methods, graph based methods, and geometry based methods.

Feature based methods depend on measuring and comparing features and are based on pure geometry of the shape. These methods can be sub-divided into:

1. Global features: global features characterize the global shape of the 3D model. Examples of these features are volume of the model, volume-to-surface ratio, area, the Fourier transform of the volume, or the boundary of the shape. A disadvantage of this method is that it does not discriminate object details well.

2. Global feature distribution: Instead of comparing global features, this method compares distributions of global features. Shape distributions are compared based on properties such as distance, angle, area, volume between random points, and shape histograms using the principal axes of inertia of the model (e.g., moment of inertia about the axis, average distance from the surface to the axis, variance of distance from the surface).

3. "Spatial maps are representations that capture the spatial location of an object. The map entries correspond to physical locations or sections of the object, and are arranged in a manner that preserves the relative positions of the features in an object. Spatial maps are in general not invariant to rotations, except for specially designed maps." (Tangelder and Veltkamp, 2008).

4. Local features: This method accounts for surface shape in the neighborhood of points on the boundary of the shape. Metrics for similarity are obtained from mapping of the surface curvature of the object onto a unit sphere, using histograms of shape indices calculated over the entire mesh, and applying semi-local descriptions of object shape centered at points on the surface of the object (3D shape context). 
Graph based methods "attempt to extract a geometric meaning from a 3D shape using a graph showing how shape components are linked together. Graph based methods can be divided into three broad categories according to the type of graph used:"

1. Model graphs: Model graph based similarity methods are applicable to 3D CAD solid models and are difficult to apply to natural shapes like humans and animals. The most common "solid model representation methods are boundary representation (B-rep) and constructive solid geometry (CSG)."

2. Reeb graphs: A Reeb graph is defined as "the quotient space of a shape $S$ and a quotient function $f$. Reeb graphs defined by a geodesic distance are suited for matching articulated objects, but they are sensitive to topological changes. Also, they cannot be applied to arbitrary meshes, because topological problems like missing faces disturb the computation of geodesic distances."

3. Skeletons: This method uses a skeletal graph that has geometric and topological information. Some researchers have combined global features and skeletal graphs.

"For graph based descriptors the complexity of the exact computation of a metric obeying the triangle inequality prevents practical application."

Geometry based methods can be categorized into:

1. View based similarity: The premise of this method is that two 3D models are similar if they are similar from all viewing angles.

2. Volumetric error based similarity: This approach is based on "calculating a volumetric error between one object and a sequence of offset hulls of the other object."

3. Weighted point set based similarity: This method is based "on shape descriptors consisting of weighted 3D points." Researchers have used weights that are based on the volume of the component, curvature, or sum of squared distances for models aligned in the same coordinate frame.

4. Deformation based similarity: This method "compare[s] a pair of 2D shapes by measuring the amount of deformation required to register the shapes exactly." Tangelder and Veltkamp commented that it would be difficult to implement this method of 3D shape matching.

\subsection{Princeton Shape Benchmark - Shillane et al. (2004)}

The Princeton Shape Benchmark (PSB) (Shilane et al., 2004) contains a database of 3D models and software tools to compare 3D shape matching algorithms. In the paper, a review of several 3D model databases was conducted and some observations of these databases were made:

- Most previous databases contain a small number of classified models.

- Most databases contain a limited range of objects. For example, some only contain household objects or only vehicles.

- The databases have a wide range of granularities in the classifications. For example "Kitchenware" and "motorcycles with 3 wheels."

- Many classifications mix function and form. For example, "buildings" and "machine." 
The PSB classification is based mainly on semantic and functional concepts (e.g., furniture and table) and secondly on shape (e.g., round tables). Other potential classifications include: grouping models based on function, grouping based on how they are manufactured (e.g., man-made vs. natural), grouped based on where they are used (e.g., office vs. home vs. outdoors), and grouping based on who uses them (e.g., adults vs. children). The PSB allows multiple classifications - an ASCII file contains a hierarchy of class names; each with a different granularity. For example, three classifications in increasing coarseness would be tables, furniture, man-made. Additionally, the benchmark attaches several geometric attributes to each model such as:

- Center of mass - the average (x,y, z) coordinates of all points on the surfaces of the polygons

- Scale - the average distance from all points on the surface of the polygons to the center of mass

- Principal axes - "eigenvectors (and associated eigenvalues) of the covariance matrix obtained by integrating the quadratic polynomials $x_{i} \cdot x_{j}$ with $x_{i} \in\{x, y, z\}$, over all points on the surfaces of all polygons."

Of the 12 benchmarks used to compare shape matching algorithms, those of interest are:

- "D2 Shape Distribution (D2): a histogram of distances between pairs of points on the surface [Osada, 2001].

- Extended Gaussian Image (EGI): a spherical function giving the distribution of surface normals [Horn, 1984].

- Shape Histogram (SHELLS): a histogram of distances from the center of mass to points on the surface [Ankerst et al., 1999].

- Shape Histogram (SECTORS): a spherical function giving the distribution of model area as a function of spherical angle [Ankerst et al., 1999].

- Shape Histogram (SECSHEL): a collection of spherical functions that give the distribution of model area as a function of radius and spherical angle [Ankerst et al., 1999].

- Voxel: a binary rasterization of the model boundary into a voxel grid.

- Spherical Extent Function (EXT): a spherical function giving the maximal distance from the center of mass as a function of spherical angle [Saupe and Vranic, 2001].

- Radialized Spherical Extent Function (REXT): a collection of spherical functions giving the maximal distance from center of mass as a function of spherical angle and radius [Vranic, 2003].

- Gaussian Euclidean Distance Transform (GEDT): a 3D function whose value at each point is given by composition of a Gaussian with the Euclidean Distance Transform of the surface [Kazhdan et al., 2003].

- Spherical Harmonic Descriptor (SHD): a rotation invariant representation of the GEDT obtained by computing the restriction of the function to concentric spheres and storing the norm of each (harmonic) frequency [Kazhdan et al., 2003].”

\subsection{Iyer et al. (2005)}

In Iyer's et al. (2005) review of 3D shape searching, shape is defined as "all the geometrical information that remains when location, scale, and rotational effects (Euclidean transformations) are filtered out from an object." Shape searching involves the determination of similarities among 3D shapes. Included in the paper are discussions of: 
- global feature-based techniques (e.g., moments, invariants, Fourier descriptors, and geometry ratios)

- manufacturing feature recognition-based techniques for CAD models

- graph-based techniques (e.g., B-Rep graph, Spectral graph, Reeb graph, skeletal graph)

- Histogram-based techniques - sample points on the surface of the 3D model and extracted characteristics from the sampled points.

o Shape histogram

- Shell model - "The space is decomposed into concentric shells around the center point. This representation is independent of rotation."

- Sector model - "The space is decomposed into sectors that emerge from the center point of the model."

- Spiderweb model - a combination of the Shell and Sector models.

o "Shape distribution represent the shape signature as a probability distribution sampled from a shape function measuring the geometric properties of a 3D model"

- A3: Measures the angles between lines joining three random points on the surface of a 3D model.

- D1: Measures the distance between a fixed point and a random point on the surface.

- D2: Measures the distance between two random points on the surface.

- D3: Measures the square root of the area of the triangle formed by three random points on the surface.

- D4: Measures the cube root of the volume of the tetrahedron formed by four random points on the surface.

- Product information-based techniques - designed specifically for engineering parts. "Part designs are described either based on their manufacturing attributes or on their geometry."

o Group technology (GT) - "coding and classification schemes attempt to capture design and manufacturing attributes such as the main shape and size features of the product, production quality, and material."

o Section image-based - uses 2D silhouette of parts or sections for similarity comparison.

- $3 \mathrm{D}$ object recognition-based techniques

o Aspect graph - consists of a multiple 2D views of a 3D model of the object as seen from various regions on a sphere encompassing the object.

o Extended Gaussian images (EGI)

o Geometric hashing - a 3D object is broken down into basic geometric features such as surface points. A set of basis points are chosen and the coordinates of all other points are determined based on the basis points and are stored in histograms. This process is repeated for other basis sets.

\subsection{Corney et al. (2002)}

Corney et al. (2002) proposed three convex-hull based indices as coarse filters in preliminary steps in the shape matching of 3D engineering data which supports the internet-based search engine ShapeShifter. Factors in selecting these indices include:

- the ability of the search engine to identify similar shapes independent of size 
- filters that resulted in an almost complete set of correct candidates (i.e., filters did not eliminate correct candidates)

- Shapeshifter employs several metrics based on the model's convex hull

Other metrics calculated in ShapeShifter include volume, surface area, aspect ratios (of an axis-aligned bounding box), crinkliness, compactness, number of facets, number of holes, and convex hull. Corney et al. used four dimensionless ratios based on the hull as filters to assess their sensitivity to object variations (scaling, taper, twist, feature removal, and fits in a $100 \times 100 \times 100$ box):

- Bounding box aspect ratio: ratio of the longest edge to the shortest edge

- Hull crumpliness: ratio of the object's surface area to the surface area of its convex hull

- Hull packing: proportion of the convex hull volume not occupied by the original object computed as $\left(1-\frac{V_{\text {model }}}{V_{\text {hull }}}\right)$

- Hull compactness: ratio of the convex hull's surface area cubed to the volume of the convex hull squared

They found that:

- the bounding box aspect ratio could potentially exclude many potential candidate matches and was thus excluded as a filter,

- hull crumpliness is relatively insensitive to twisting and warps and sensitive to feature removal and non-uniform scaling,

- hull packing is insensitive to all but feature removal, and

- hull compactness is relatively insensitive to tapering, is sensitive to twisting and non-uniform scaling, and insensitive to feature removal.

For the three filters based on the convex hull, the optimal tolerance values were $25 \%$ for compactness, $50 \%$ for packing, and $10 \%$ for crumpliness where optimality is based on minimizing the false-negatives and false-positives.

\subsection{Jayanti et al. (2006)}

Jayanti et al. (2006) developed a benchmark dataset of engineering artifacts to enable evaluations of various shape descriptors for engineering shape retrieval. Some challenges in developing the benchmark:

- a function-based classification is difficult because parts with similar shapes have different functions

- a classification based on manufacturing process is complicated by advances in manufacturing processes and capabilities which blurs the mapping between form and manufacturing method

The classification of 3D models in the engineering shape benchmark (ESB) is based on shape. The ESB contains 867 3D CAD models in STereoLithography ${ }^{1}$ (STL) and Wavefront Technologies' OBJ formats, and is available from http://purdue.edu/shapelab. The models are initially put into three super-classes:

\footnotetext{
${ }^{1}$ Also known as Standard Tessellation Library
} 
- Solids of revolution - part envelope is largely a solid of revolution

- Rectangular-cubic prism or prismatic - part envelope is largely a rectangular or cubic prism

- Thin-walled - parts with thin-walled sections and shell-like components

These super-classes were then further divided into groups of similar shapes. For example, clusters under solids of revolution included bolt-like parts, container-like parts, cylindrical parts, and discs. The authors used the following shape representations for their benchmarking:

- Feature vector-based methods

o Moment invariants (MI)

o Principal moments (PM)

o 3D spherical harmonics ( $\mathrm{SH}$ )

o Surface and area based attributes

- Surface area and volume (SAV)

- Surface area to volume ratio (SVR)

- Crinkliness and compactness (CC)

o Geometric ratios (GR) - aspect ratios of the bounding box

- $\quad 3 \mathrm{D}$ shape distribution (3DSD) using the D2 shape measure

- Convex hull histogram (CHH)

- 3D shape histogram - solid angle (SAH)

- View-based representations

o Light field descriptors (LFD)

o 2.5D spherical harmonics (2.5DSH)

o 2D shape distributions (2DSD)

The reader is referred to the paper for detailed descriptions of these shape representations.

Precision-recall curves were used to evaluate these shape representations. "Precision is the ratio of the relevant models retrieved to the retrieval size. Recall is the fraction of the relevant models retrieved for a given retrieval size." Some conclusions based on the results using the ESB are that the three methods based on 2D views (LFD, 2.5DSH, and 2DSD) consistently outperformed the other methods, with the LFD resulting in significantly better precision compared to all other methods. However, view-based methods require more computation as compared to 3D shape measures. 3D spherical harmonics ( $\mathrm{SH}$ ) perform reasonably well for the prismatic parts and solids of revolutions as compared to other shape representations. In terms of the three super-classes:

- Thin-walled components

o View-based methods performed better than other methods

o 3D shape distributions, surface area, and volume performed better than more complex feature vectors - $\mathrm{SH}, \mathrm{CHH}$, and $\mathrm{SAH}$

o SAH performs better for this class than other point-based methods

0 Of the view-based methods (LFD, 2.5DSH, and 2DSD), all three methods performed comparably

- Prismatic parts

o LFD had significantly high precision for all recall levels 
o 2.5DSH, 2DSD, SH, and SAH consistently performed better than the 3DSD method

o Topology-based methods did not perform well especially at lower recall values

- Solids of revolution

o All view-based methods (LFD, 2.5DSH, 2DSD) performed significantly better than the other methods

o 3DSH and $\mathrm{CHH}$ performed better than 3DSD

\subsection{Cardone et al. (2003)}

Cardone et al. (2003) conducted a survey of shape similarity assessment algorithms for product design and manufacturing applications. 3D similarity assessment is described as the generation of shape signatures from the 3D models and comparing them using distance functions; the larger the value, the more dissimilar. The shape signatures should be "representation independent and completely describe the features of the 3D model." Examples of shape signatures are graphs, vectors, or ordered collections of numeric values. The choice of signatures is dependent on the reason for the similarity analysis. Shape signatures and distance functions should have the following properties:

- Positivity - distance between two CAD models should be non-negative

- Identity (Self-similarity) - if the distance between two CAD models is zero, then the models are the same

- Symmetry - given solid models $x$ and $y$ with shape signatures $\mathrm{S}(x)$ and $\mathrm{S}(y)$, respectively, a symmetric distance function needs to satisfy:

$\delta(\mathrm{S}(x), \mathrm{S}(y))=\delta(\mathrm{S}(y), \mathrm{S}(x))$

- Triangle inequality - given solid models $\mathrm{x}, \mathrm{y}$, and $\mathrm{z}$ with shape signatures $\mathrm{S}(x), \mathrm{S}(y)$, and $\mathrm{S}(z)$, respectively, the distance function must satisfy:

$\delta(\mathrm{S}(x), \mathrm{S}(y))+\delta(\mathrm{S}(y), \mathrm{S}(\mathrm{z})) \geq \delta(\mathrm{S}(x), \mathrm{S}(z))$

- Invariance - a part may have many different representations; "the shape signature should be invariant with respect to the underlying representation." The shape signature should also be invariant to any rotation or translation applied to the object.

- Robustness and sensitivity - shape signature should change proportionately to the change in the object. If small changes are magnified and result in the conclusion that similar objects are dissimilar, then the measure is not robust. If there is a large change in the object which results in a small change in the shape signature, then it is not sensitive.

- Computational efficiency

The similarity assessment techniques were grouped into:

- Features: "Feature-based techniques evaluate the shape signature of an object based on the type, size, orientation, number, and other properties of the features and their interactions. Once the features are extracted and their significant characteristics are determined, the comparison is carried out using a suitable distance function.” Disadvantages include that the technique does not consider the gross shape of object and feature interactions complicate feature extraction. Techniques described in paper were developed for product design and manufacturing.

- Spatial functions: "These techniques use shape signatures that are spatial functions. An example of a spatial function is the Gaussian map that maps the set of normals of a solid onto a unit 
sphere. The problem of matching and comparing 2D spatial functions defined over unit sphere involves manipulating two degrees of freedom (the two angles needed to align the surface of a sphere). The main challenge in these techniques is to identify the characteristics to be represented using spatial functions and to determine an efficient matching procedure to compare two shape signatures."

o Local curvature distributions over mesh representations: the signature is the spherical representation of the curvature distribution of 3D surfaces of solids without holes. This technique is useful for applications where curvature is a primary factor. Advantages include that the measure is invariant to translation, rotation and scaling. Disadvantages are the restriction to solids without holes and that accuracy depends on the mesh size - a finer mesh increases accuracy but increases computational time.

o Slope diagram representations: this technique uses the slope diagram representation of a convex polyhedron. It is invariant to translation and rotation but computationally intensive and restricted to convex polyhedra.

- Shape histograms: "These techniques are based on sampling of points on the surface of the 3D models. Several significant characteristics can be extracted from the set of points obtained." The larger the number of points, the more accurate the representation, but the more expensive it is to compute. These techniques do not satisfy the conditions of identity and symmetry but are useful for detecting gross shape similarity, that is, useful as coarse filters.

Several shape functions were described in the paper but the most robust of these was the D2 shape function.

o D2 shape function "computes the distance between two random points. This function is invariant to rotation and translation and is robust." The distances between random points are calculated and normalized using the mean distance. "The shape distribution is the histogram that measures the frequency of occurrence of distances within a specified range of distance values." The distance between the two models is usually computed using the L2 norm.

- Section images: "These techniques use sections of the solids as shape signatures. Solids are sectioned at various places and the sections are then analyzed for similarity. A disadvantage is that the method is not invariant to scaling, translation, and rotation. It is best suited for parts that are rotationally symmetric."

- Topological graphs: "These techniques use topological graphs as shape signatures. These graphs usually represent the connectivity information of the boundary of the solid such as the adjacency between faces. The nodes and edges of the graph may carry additional information related to the solid model."

- Shape statistics: Shape comparison techniques commonly use basic geometric properties for coarse comparison between objects. Some commonly used properties are volume, surface area, and convex hull volume. Disadvantages include that the measures do not yield sufficient information for detailed comparison, but can be used as coarse filters.

A table comparing the representative algorithms is given in the paper. 


\subsection{Bespalov et al. (2005)}

Bespalov et al. (2005) used several benchmark datasets to evaluate nine techniques for matching CAD data. CAD models can either be solid models or shape models represented by polygonal meshes. The increasing use of laser scanners has made the generation of polygonal meshes a relatively easy task. The matching techniques evaluated were:

- Shape based

o Shape distributions (SD) (Osada et al., 2002)

o Shape distributions with point pair classifications (SD-class) (Ip et al., 2002)

o Reeb graph comparison (Reeb) (Hilaga et al. 2001)

o Shape distributions with weight learning (SD-Learn) (Ip et al., 2003)

o Scale-Space comparison (Scale-Space) (Bespalov et al., 2003)

- Solid based

o B-Rep techniques

- Invariant topological vector (ITV) (McWherter et al., 2001)

- Eigenspace indexing on B-Rep graphs (Eigen-BRep) (Peabody 2002)

o Feature based

- Model dependency graph approximate matching (MDG) (Cicirello and Regli 2002)

- Eigenspace indexing on matching feature interaction graphs (Eigen-Feat) (Peabody 2002)

The $k$-nearest neighbor classification $(k N N)$ and conventional recall and precision measures were used to evaluate the techniques. Recall and precision were defined as:

$$
\begin{aligned}
& \text { recall }=\frac{\text { Retrieved and relevant models }}{\text { Relevant models }} \\
& \text { precision }=\frac{\text { Retrieved and relevant models }}{\text { Retrieved models }},
\end{aligned}
$$

where

relevant models was "the number of models that fall into the same category as the query model" retrieved models was "the number of models returned by a query"

retrieved and relevant models was "the number of models returned and that fell into the same category as the query model."

The datasets used to evaluate the techniques were:

- Synthetic datasets

o Primitive dataset: 101 cubes, 141 cylinders, 29 tori, and 29 spheres with various deformations.

o Minor topological variation dataset

- cube-holes: 16 cubes with different numbers of holes (1 to 4)-some with holes of different radii and some with holes of the same radius 
- brick-holes: 11 rectangular box models with 0 to 4 holes of the same size in different locations.

- Actual artifact dataset:

o CAD models from the National Design Repository Dataset (http://edge.cs.drexel.edu/repository/) were used.

- Manufacturing classification:

- prismatic machined (56 models) - machined parts are typically high precision parts made in small batches

- cast-then-machined (54 models) - cast-then machined parts are typically larger production jobs with less stringent tolerance requirements

- Functional classification: 70 models total, example functional classes: nuts, gears, screws, springs, linkage arms.

o LEGO Dataset - 47 components grouped by appearance: plates, wheels and gears, cylindrical parts, and X-shaped axles.

o Variable fidelity dataset - 40 CAD models were used to generate 40 polygonal meshes each of low, normal, and high fidelity (highest number of polygons), i.e., 120 models total.

Some findings and observations from the evaluation:

- The graph and solid based ITV and Eigen-BRep techniques performed best on the cube-holes and brick-holes datasets. Among the shape based techniques, the Reeb graph technique performed best.

- Boundary representations (B-Reps) are more useful than mesh-based representations and improving ways to better use B-Reps for matching would be beneficial

- Based on the precision-recall plots, the manufacturing and functional classifications yielded unacceptable and "mediocre" results, respectively, for all the techniques. 


\section{Object Classification for Perception Systems}

As stated in the introduction, our purpose for developing an object classification is to evaluate perception systems, agnostic to the nature and details of the system. This is different from the purpose of the classification systems developed by most researchers in Section 2, where the intent typically was to develop a perception system or to evaluate specific algorithms. The purpose for the system developed by Jayanti et al. (2006) is closest to our purpose, with an analysis that relates general classes of objects to the effectiveness of particular algorithms.

Ideally, our proposed classification system would put objects into classes where the objects in a class would yield similar results, good or bad, from the performance of a wide selection of perception systems. A particular class of objects might be easy to perceive (e.g., textured boxes), or difficult (highly curved, specular parts). Ideally this would be sensor agnostic, so an easy to perceive class of objects remains easy to perceive across all perception systems, but in practice there will remain dependences on the nature of the sensor and perception algorithm. A passive sensor attuned to color will work well in distinguishing identically shaped but differently colored objects, while a monochrome active sensor attuned to shape may do better with color-less but differently shaped objects. The classification system would then be useful in specifying which class of object is most easily perceived by which type of sensor system.

Several concepts for developing a classification system were considered. These included classification systems based on:

1. Technology of the perception system, such as these examples:

a. Laser line scanner

b. Machine vision (with one or more cameras)

c. Structured light/pattern projection (may be visible or non-visible)

2. Objects with similar features or descriptors

3. A priori knowledge of issues for perception systems based on feedback from vendors, integrators, and users on objects that were problematic or not problematic for their perception systems

A logical choice would be classification based on perception system technology, giving up sensoragnostic classification in return for more effective, technology-specific categorizations. However, this would require acquiring intimate knowledge of the various types of perception systems and the availability and extensive evaluation of these systems to determine the advantages and disadvantages of systems that represented each of the various technologies. Given budgetary and time constraints, a decision was made that, as a first step, the classification system would be based on object characteristics and the authors' knowledge and experience with the various technologies.

The authors felt that a classification system that encompasses all manufacturing objects would not be feasible, and a narrowing of the types of objects was needed. Since the focus of the project was for bin picking applications, it was decided that the classification system would initially be developed for rigid, automotive parts found in the engine compartment and parts in the powertrain. The maximum size of the part/object was therefore set, arbitrarily, to be $(50 \times 50 \times 50) \mathrm{cm}$. 
Given the above discussion, we compiled a set of metrics that were used by other researchers to characterize an object. By metrics we mean features of the object, local or global, that can be used to classify it. When possible, we emphasized quantitative features. The full set of metrics is given in Table $\mathrm{C} 1$ in Appendix C. Table $\mathrm{C} 1$ has the following information for each metric:

- Feature: Name of feature

- Local feature: a characteristic of the object computed on a limited, local region

- Global feature: a characteristic of the object computed with global support.

- 2D/3D/Both: Whether the feature is primarily two dimensional, three dimensional, or both.

- Shape/Surface: Whether the feature applies primarily to the shape or surface of the object.

- Description: Description of the metric to be computed.

- Relevant or Non-Relevant: Whether the metric is relevant to our classification task, based on the judgment of the project team members.

- Algorithm/software needed? Y/N; Available? This column indicates whether an algorithm or software is required to compute the metric. If an algorithm or software is needed, is it available?

- Source: Reference(s) in which the metric was invented or described.

The metrics were chosen to describe a broad range of perceptual characteristics of objects, including:

- Geometry: metric properties of shape (Table 3.1)

- Topology: relationship of components (Table 3.1)

- Viewpoint projections/appearance: features computed on 2D projections of the part (Table 3.2)

- Surface properties: color, texture and other (Table 3.3)

The metrics do not provide a complete physical description of an object, as mass and material characteristics, aside from appearance, are not included. We were assuming that sensors would be primarily non-contact and utilize visual or near-infrared (IR) wavelengths and not utilize thermal IR bands, sonic wavelengths, magnetic, weight, or other non-visual physical properties. Also, a given shape described by a CAD model can be manufactured from different materials, which can result in different physical properties (including surface reflectance). Our primary goal was to consider those aspects of an object captured by shape encoded in a CAD model and that would be found in the object even if made with different materials.

For the geometric metrics in Table 3.1, we selected a range of object characteristics that could be used either directly as a perceptual feature, or are likely to contribute to the appearance of perceptual features. An example would be whether the object is prismatic, with a number of flat surfaces that might lead to internal visual edges in an image, or rounded, with less likelihood of internal edges aside from folds and limbs. We were inclusive in the metrics, cataloging many with differing amounts of information rather than focusing on a few, high information features. The intent was not to build an efficient perception system, but rather to understand how the metrics might partition the space of objects.

Some geometrical metrics were selected not because they alone represented a visual feature, but because they may indicate the presence of visual features. In a manufacturing application, the problem of object 
identification may require relatively simple discriminations, such as between a long thin object and a more compact one, so simple extents would be adequate. Similarly, object density (here not density of mass, but of shape as represented by the ratio between the volume of the object and the volume of its convex hull) could be a marker for relatively hollow objects, like springs, that are likely to have selfocclusions.

\section{Table 3.1. List of Selected Metrics for Object Classification.}

\begin{tabular}{|c|c|c|c|}
\hline & Metric & Metric Measured & Comments \\
\hline 1 & Size & $\mathrm{X}, \mathrm{Y}, \mathrm{Z}$ extents & \\
\hline 2 & Size & Surface area & \\
\hline 3 & Size & Volume & \\
\hline 4 & Size & Convex hull & \\
\hline 5 & Compactness & Volume/Surface Area & \\
\hline 6 & Compactness & Max/Min extent & \\
\hline 7 & Compactness & $(\text { Area of Convex Hull })^{3} /(\text { Volume of Convex Hull })^{2}$ & \\
\hline 8 & Density & 1-Volume/(Volume of convex hull) & \\
\hline 9 & Flatness & Short/Middle extent & \\
\hline 10 & Flatness & Middle/Long extent & \\
\hline 11 & Prismatic & $\%$ surface flat & \\
\hline 12 & Curvature & Integrated total curvature & \\
\hline 13 & Curvature & $\%$ convex, concave, saddle & \\
\hline 14 & $\begin{array}{l}\text { 3D } \\
\text { Uniqueness }\end{array}$ & \# significant feature points & $\begin{array}{l}\text { Use SURF }^{2} \text { or SIFT } \\
\text { this metric (D get } \\
\text { 2012) }\end{array}$ \\
\hline 15 & Symmetry & Rotational moments & \\
\hline 16 & Symmetry & \# axes+type & Mirror, rotational, point \\
\hline 17 & $\begin{array}{l}\text { Anti- } \\
\text { symmetry }\end{array}$ & Chirality & \\
\hline 18 & Smoothness & $\begin{array}{l}\text { Hull crumpliness: ratio of object surface area to surface area } \\
\text { of its convex hull }\end{array}$ & \\
\hline 19 & Smoothness & $\begin{array}{l}\text { Crinkliness: defined as the surface area of the model divided } \\
\text { by the surface area of a sphere having the same volume as } \\
\text { the model. }\end{array}$ & \\
\hline 20 & Topology & \# holes & \\
\hline
\end{tabular}

Another reason for selection of some metrics is the performance of current perception systems. Many bin picking systems perform well on symmetric objects, such as discs and cylinders. The performance may be related to ease of perception, or ease of grasping, but in either case symmetry is an important metric.

Reviewing the metrics in Table 3.1, the first set (1-10 in Table 3.1) was selected to classify the compactness and extent of the object. The $\mathrm{x}, \mathrm{y}, \mathrm{z}$ extents, surface area and volume are basic measures of the object size, which are not directly useful in perception unless sensor distance or field-of-view is taken into account. Metric 4, convex hull, is also less useful directly but is useful as an intermediate computation for other metrics.

2 Speeded Up Robust Features

${ }^{3}$ Scale-Invariant Feature Transform 
The compactness metrics (5-7 in Table 3.1), as well as the global flatness metrics (9-10), measure ratios that estimate whether the object is compact in all three dimensions (metrics 5-7), or in one dimension and is therefore flat (metrics 9-10). Compactness alone may not be significant in ease of perception, but indirectly may influence other features. A flat object yields fewer possible poses and may result in effectively a 2D shape easy to perceive by traditional machine vision approaches.

The density metric ( 8 in Table 3.1) measures the open volume of an object. The logic is: if the object is compact and dense, the volume of the convex hull will be close to the volume of the original object and the ratio would be close to 1 , so the density measure will be 0 . If the object is very open inside, or has long extensions, the volume of the convex hull will be greater than the original volume, so the ratio will be smaller and the density measure larger. A density measure near 1 could indicate a visually hollow object, like a spring, which is likely to have self-occlusions and other distinct visual characteristics. (Some objects may be hollow, like a box or a gas tank, but look solid from the outside - in this case the metric may misfire, or need to be properly interpreted.)

The second set of metrics (11-13 in Table 3.1) was selected to classify surface roundness and curvature. These measures have long been used in object description and perception, but are hard to describe in single numbers for an entire object. Curvature is a local feature (e.g., for a meshed object, the curvature is calculated at a vertex), and an object can have a highly curved element attached to a flat section. We looked for measures that would help distinguish a relatively flat object, like a box, from a highly curved object, like a ball bearing or a section of pipe. Our measures all essentially integrate the local curvature. For prismatic shapes (metric 11), the measure is the percentage of the shape that is locally flat with zero curvature. For more curved shapes we consider metric 12, the integrated total curvature (e.g., for a meshed object, the curvature averaged over all vertices). This is intended to give the average curvature over the entire object. Finally, metric 13 is intended to measure the percentage of the object that is locally convex or concave.

The third set of metrics (14 in Table 3.1) was selected to identify objects with many distinct 3D, highcurvature local features. This would distinguish homogenous objects like a sphere from heterogeneous objects with many local dips, angles, and extensions, like the body of a fuel pump. In this case, there are many different $3 \mathrm{D}$ feature measures that could be used, but we determined that most measures would give highly correlated results at the level of discrimination we need. If an object has a large number of distinctive corners or bumps found by one measure, another measure is likely to also find many features even if not the same ones.

The fourth set of metrics (15-17 in Table 3.1) was selected to measure symmetry. Symmetry is a complex concept that can be difficult to quantify on a CAD model, so the metrics were difficult to choose (and compute). Shapes can be symmetric by rotation, by reflection, by a helix, and other mechanisms. To categorize a shape's symmetry, the nature of its symmetries has to be discovered and quantified for imperfect symmetries. We found the symmetry characteristic difficult to quantify and to compute, and did not arrive at metrics we found adequate to fully and appropriately describe symmetry. Still, symmetry can be important in perceptual systems - symmetric objects allow matching by techniques like generalized cylinders, and simple circular shapes admit to fast detectors.

The fifth set of metrics (18-19 in Table 3.1) was selected to measure surface smoothness (roughness), which influences the appearance of color and texture. The two metrics essentially measure the ratio of the 
object's surface area to the surface area of an idealized version of the object (convex hull or sphere), and if the ratio is high, the object should have folds or other high-frequency variations in its surface.

The sixth and last class of metrics (20 in Table 3.1) was selected as a marker for topology. The measure we selected was the genus of the surface, or the number of holes invariant to plastic distortions. A sphere has zero, a torus one, and so on. These are holes completely though the object. In this case, we are not certain that the number of holes an object has will be significant in characterizing its appearance. A small, hard-to-see hole drilled though the object will increase the genus just as much as a large, distinguishing hole. We did not select metrics related to the branching or subpart relations of an object, which are sometimes described as topological. These can be complex and are beyond the scope of this study.

The metrics in Table 3.2 were selected to determine the viewpoint-dependent features of an object. There should be some correlation between the viewpoint-independent shape metrics in Table 3.1 and the viewpoint-dependent metrics in Table 3.2, since density, curvature, and smoothness may influence the nature and number of local 2D features in 2D projections. However, we also wanted a direct measure of the number and distinctiveness of $2 \mathrm{D}$ features across possible viewpoints. The more distinctive $2 \mathrm{D}$ features, the more likely an object can be identified and located in 2D projective sensors (e.g., cameras).

Table 3.2. Metrics Based on Pose or Viewpoint.

\begin{tabular}{|c|l|l|l|}
\hline & \multicolumn{1}{|c|}{ Metric } & \multicolumn{1}{|c|}{ Metric Measured } & \multicolumn{1}{c|}{ Comments } \\
\hline 1 & Poses & \# stable poses & See Yee, Wan, Ventura 2001 \\
\hline 2 & Unique views & Visually distinguishable poses & \\
\hline 3 & 2D Uniqueness & \# significant 2D feature points & \\
\hline
\end{tabular}

However, the metrics in Table 3.2 require considerable effort to compute. To properly do so, many views of the object have to be systematically produced, with the views simulating the camera or sensor of interest, and each view analyzed for the number of distinct feature points. Similarly, to determine the number of stable poses requires a physical simulation of the object. Also, the simulated 2D views will change if the object has texture.

The metrics in Table 3.3 are material, color, texture, and reflectivity. These were selected for their obvious influence on object appearance. However, we did not emphasize these in the study for a few reasons. The first is that CAD models do not typically represent inherent surface properties, so shape is paramount. The second is that in manufacturing assembly, many parts do not have distinctive texture or color. They can be machined metal or texture-less plastic, used in internal assemblies where markings are not essential. The third is that surface textures can be arbitrary and highly varying, such as a single shape of a soft drink can that may be printed with many variations of logos and brand names. Finally, object appearance can vary over the object, face by face or section by section - should objects be classified on average appearance, maximum texture, a histogram of color or texture metrics, or some other way? Classifying appearance and textures for ease of perception would be another complete study. Finally, once color or texture is introduced into perception for a manufacturing application, there are likely to be existing, successful machine vision approaches for object identification. 
Table 3.3. Surface Appearance Metrics.

\begin{tabular}{|l|l|l|}
\hline & \multicolumn{1}{|c|}{ Metric } & \multicolumn{1}{c|}{ Metric } \\
\hline 1 & Reflectance & Bidirectional reflectance distribution function (BRFD) \\
\hline 2 & Object material & As given in object description \\
\hline 3 & Color & Metric is appropriate color or radiometric space; possibly sensor dependent \\
\hline 4 & Texture & Many possible structural, statistical and power spectrum metrics \\
\hline
\end{tabular}

\section{Creation of an Object Database}

The calculation of the proposed metrics for an object requires a 3D representation of that object. An initial strategy was to try to obtain CAD models of engine parts (from older or discontinued auto models) from the various automobile manufacturers or from free sources on the web. Both Ford Motor Company and General Motors were contacted, and unfortunately, this strategy was not viable due to proprietary issues. The latter option of finding engine parts on the web required developing a list of engine part names and few, free CAD models of engine parts were found.

An alternative was to use the models available in the Purdue Engineering Shape Benchmark (ESB) (https://engineering.purdue.edu/cdesign/wp/) database that were deemed similar to engine parts. The database contained images of the part, an OBJ file for the part, and a mesh of the part in STL format. A subset of about 200 objects from the over 900 objects were selected from the ESB database. Metrics based on geometry were calculated for the selected parts. However, the results from the curvature algorithm did not yield rational results. The reason for this was because a finer mesh was required. An attempt was made to create finer meshes of the parts using the OBJ files of the objects that were available from the Purdue ESB database, but we were unable to open these files with any of the commercially available software that we had.

As a result, another web search of freely available CAD models was conducted. This search yielded over a hundred parts from the following website: www.GrabCAD.com. The restriction of parts associated with automobile engine parts and the availability of usable file formats (i.e., file formats for which the authors had software to open the files), limited the selection of objects. As stated in Section 3, objects that fit within a $(50 \times 50 \times 50) \mathrm{cm}$ volume were used in the analysis.

Another option that arose was the purchase of CAD models, such as of an automotive engine. However, there were a few of drawbacks to this option: cost, procurement time, and type of file. The cost was not prohibitive as CAD models of an engine are available for about $\$ 600$. The files for the parts were meshed files, and they did not appear to be fine meshes. This issue may have been resolved by requesting the polygonal models of the parts, which would allow us to re-mesh to finer meshes, but we did not get a response from the creator of the mesh files.

Therefore, the objects used for testing the metrics and classification system were those obtained from the GrabCAD website and are given in Appendix A. One hundred and thirty-three (133) objects were downloaded; however, two of the objects could not be meshed and curvature metrics could not be performed for another 15 of the objects. There are some known bugs in the curvature algorithm and 
further debugging of the algorithm was not pursued as this was beyond the scope of this effort. This reduced the object database to 116. Six of the objects had at least one dimension larger than $50 \mathrm{~cm}$. These objects were left in the database as it did not have a significant effect on the manual classifications or the cluster-based classifications. However, when plotting the metrics for the High/Low observations, the metrics for the six oversized objects were excluded; thus, these observations were based only on objects that were $(50 \times 50 \times 50) \mathrm{cm}$ or smaller. 


\section{Computation of Metrics}

Different metrics characterizing an object were computed using a triangulated mesh of the object. Meshes of the objects, in STL format, were created from CAD models of the objects. The STL files were then converted to PLY (Polygon File Format) files. A direct generation of PLY files from the CAD models was not done as the generated PLY meshes were not always triangulated. The conversion from STL to PLY format was done to remove duplicate nodes.

The mesh files (in PLY format) were processed using a custom script written in MATLAB. Some functions used in the script were downloaded from the publicly available toolbox_graph which contains procedures for processing mesh data, http://www.mathworks.fr/matlabcentral/fileexchange/5355-toolboxgraph/content/toolbox_graph/html/content.html (last accessed: 08/30/13). Below are two lists of metrics: a list of primary characteristics calculated directly using the mesh of an object and a list of characteristics derived from the primary characteristics. Each metric is briefly described below.

A. Primary characteristics

1) Total surface area of an object: this is the sum of the areas of all triangles. The area of a single triangle was calculated as one half of the length of the cross product of two vectors forming two sides of a triangle. NOTE: the surface of the selected objects (see Section 4) had to be represented by one continuous and closed mesh. This restriction excludes CAD models of objects built from distinct subcomponents with more than one surface.

2) Total volume of an object: the algorithm is based on the method described in Zhang and Chen (2001). NOTE: the function which calculates the volume assumes that all normals are correctly oriented, i.e., pointing outward.

3) X, Y, Z extents (DX, DY, DZ): The maximum extents along the $x-, y-$ and $z$-axes in which the CAD model was created. NOTE: the three extents are not necessarily equal to the dimensions of the bounding box containing the object. However, in most cases, the coordinate frame of the $\mathrm{CAD}$ model is aligned with the natural directions associated with the object, e.g., axes of symmetry or three longest edges.

4) Area of the convex hull. The MATLAB function convhull() was used to generate a mesh representation of the convex hull of the object. The total surface area of the convex hull was then computed using the same procedure used to compute the total surface area.

5) Volume of the convex hull. The same algorithm used to compute the total volume was used on the mesh created with MATLAB function convhull() to obtain the convex hull volume.

6) Fraction of an object's surface area having a given shape type. Shape type is determined from the principal curvatures $\left(c_{\min }, c_{\max }\right)$ computed at a node. The principal curvatures for the six shape types are given in Table 5.1. In Table 5.1, the notation within the parentheses indicate whether $\left(c_{\min }, c_{\max }\right)$ are positive, negative, or zero. 
Table 5.1. Shape types and associated principal curvatures.

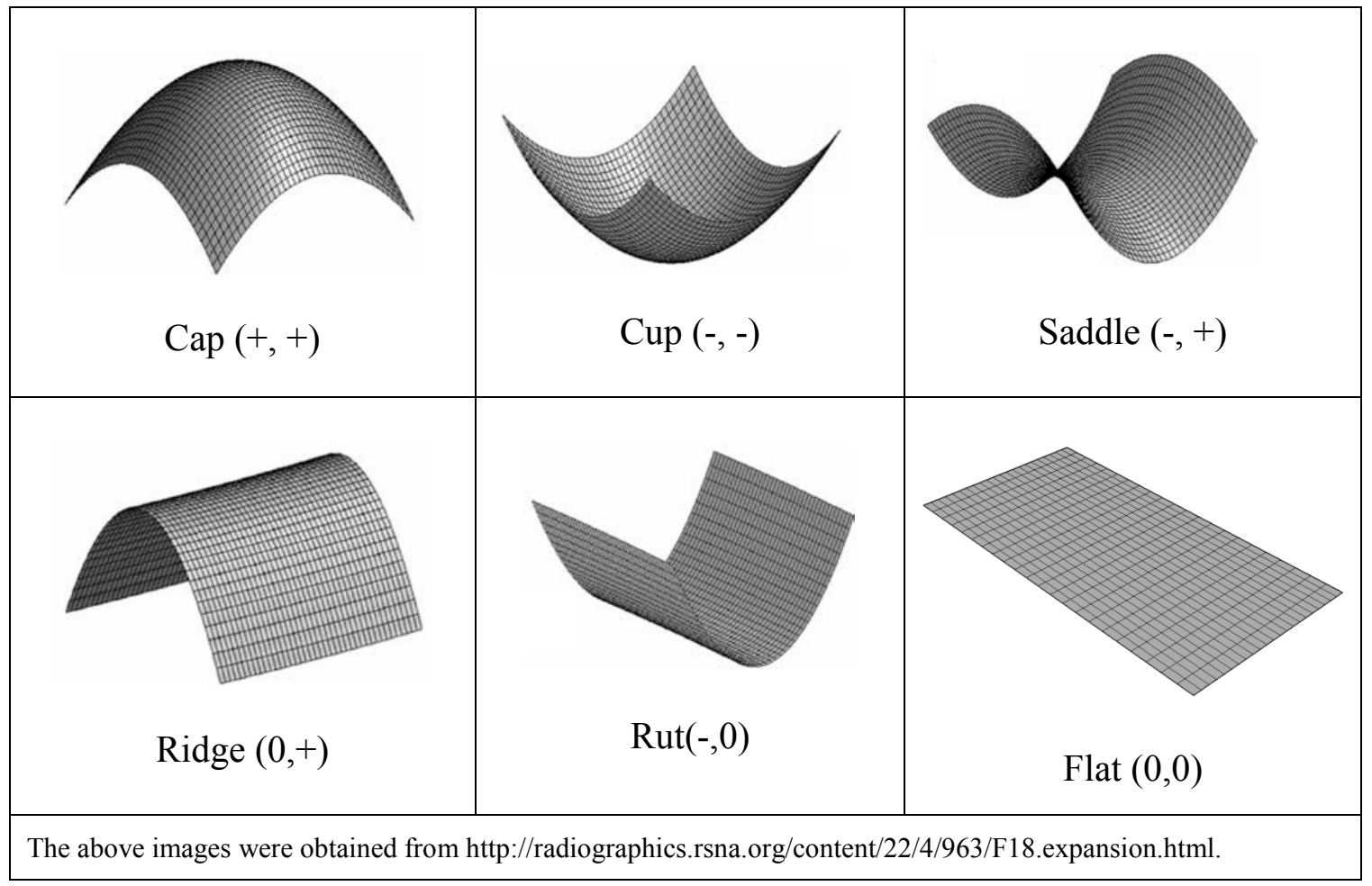

The curvature tensor was calculated by calling the function compute_curvature() from toolbox_graph. This function calculates the tensor at every vertex. Then, the curvature tensor for a triangle is calculated as the average of the tensors for the three vertices that define the triangle. The shape type of a triangle is determined by the average tensor. The area of all triangles with the same shape type are summed up and normalized by the total surface area of the object. The algorithm for calculating the curvature is based on the method developed by Alliez et al. (2003) and Cohen-Steiner and Morvan (2003). The method calculates the angular difference between the normals of all triangles which share the same vertex for which the curvature tensor was calculated.

NOTES: i) The computed curvatures will likely not have values exactly equal to zero, i.e., a flat surface. Therefore, a parameter called the flatness threshold was defined to determine whether the vertex was on a flat region or not. A meshed cube, sphere, and cylinder were used in simulations to determine a value for the flatness threshold. Based on the simulations, the flatness threshold value was set to 0.001. ii) Principal curvatures have dimension of (1/length) and are sensitive to scale. However, the function compute_curvature() outputs results which are scale invariant because the code normalizes the length of each triangle edge by the average length of all edges (for example, the curvatures of a sphere do not depend on the radius of a sphere). Normalization does not affect the shape type classification - in fact, normalization bounds the calculated curvatures for all the objects in a narrow range, making it easier to determine the flatness threshold. iii) The function compute_curvature() may sometimes fail as it did in 15 of 131 cases. The reason for this is unknown and this bug was 
reported a few years ago but was not fixed ${ }^{4}$. iv) Since the mesh obtained from a CAD model does not contain any noise (only discretization error due to meshing), the parameter option.curvature_smoothing, in the function compute_curvature(), was set to 1. Basically, this means that no averaging was applied.

7) Total length of sharp edges. An edge is classified as sharp if the directions of the two normals of the adjacent triangles differ by more than $45^{\circ}$. The reported value is the sum of all sharp edges divided by the sum of the maximum extents (calculated in 3 above). Although, this metric is not in Table 3.1, we could easily calculate this metric since we calculated the shape type in (6) and we felt that it could be a useful metric. For laser-based perception systems, a higher value for this metric may indicate potentially noisier data due to edge effects (i.e., splitting of the laser beam at edges).

NOTE: normalization makes the comparison between different objects scale invariant, for example the normalized length of a cube is 4 , regardless of the size of the cube. The normalized lengths for some geometrical primitives are given in Table 5.2.

Table 5.2. Normalized length of sharp edges for some geometrical primitives.

\begin{tabular}{|c|c|}
\hline Geometrical primitive & Value \\
\hline Sphere & 0 \\
\hline Cube & $\frac{\pi}{\left(1+\frac{H}{2 D}\right)}$ \\
\hline $\begin{array}{c}\text { Cylinder } \\
\text { with diameter } D \text { and height } H\end{array}$ & $\frac{2 \pi\left(\frac{D_{\text {in }}}{D_{\text {out }}}+1\right)}{\left(2+\frac{H}{D_{\text {out }}}\right)}$ \\
\hline $\begin{array}{c}\text { Cylindrical ring } \\
\text { with inner diameter } D_{\text {in }}, \text { and outer } \\
\text { diameter } D_{\text {out }}, \text { and height } H\end{array}$ & $\frac{\pi}{\left(2+\frac{H}{D}\right)}$ \\
\hline $\begin{array}{c}\text { Circular cone } \\
\text { with diameter } D \text { and height } H\end{array}$ & $4\left[1+\sqrt{\left(\frac{H}{L}\right)^{2}+0.5}\right]$ \\
\hline $\begin{array}{c}\text { Square pyramid } \\
\text { with side } L \text { and height } H\end{array}$ & $\frac{\left(1+\frac{H}{L}\right)}{2}$ \\
\hline
\end{tabular}

B. Derived characteristics

1.) In the literature, we found three definitions for compactness (we used all three definitions in this study):

a. Volume/Surface area

\footnotetext{
${ }^{4}$ See http://stackoverflow.com/questions/12922419/rectifying-compute-curvature-m-error-in-toolbox-graph-inmatlab and http://www.mathworks.com/matlabcentral/fileexchange/5355-toolbox-graph, Post 03 Mar 2009 by David S.
} 
b. Max extent/Min extent

c. Convex hull area ${ }^{3} /$ Convex hull volume ${ }^{2}$

2.) Density $=(1-$ object volume/convex hull volume $)$

3.) Smoothness

a. Hull crumpliness $=$ Object surface area/Convex hull area

b. Crinkliness $=$ Surface area/(Surface area of a sphere with the same volume as the object)

4.) Aspect ratio
a. $\mathrm{DX} / \mathrm{DY}$
b. $\mathrm{DX} / \mathrm{DZ}$
c. $\mathrm{DY} / \mathrm{DZ}$

The above metrics were calculated for all the objects in Appendix A and the calculated values are tabulated in Appendix B. In Table B1, the summation of the values for Cap, Ridge, Cup, Flat, Saddle, and Rut should equal 1 as the values represent the fraction of the object that is considered to be cap, cup, flat, ridge, rut, or saddle.

The metrics associated with symmetry were not computed because we could not find algorithms to adequately quantify symmetry. We also felt that the reason symmetry is important to perception is because objects that are more symmetric result in more pose ambiguities than asymmetric objects. Therefore, the metric "unique views" would be able to capture that aspect of an object without having to compute the "symmetry" of the object. Also, the number of significant features was not computed due to time constraints. Topological features such as the number of holes was also not obtained due to:

- Definition of a hole: would a hole that did not go entirely through the object be considered a hole? For perception systems, an incomplete hole can appear the same as a through hole, but topologically is not the same.

- Size of hole. If a perception system could not identify a hole due to poor resolution, or the hole is not readily sensed from the exterior, then would the number of holes be a relevant metric?

- Hole count is global. The topological number of a shape is a global feature that may not be sensed from any particular sensor view. 


\section{Classifications}

There were several different methods used to classify the objects in the database given in Appendix A using the metrics described in Section 5. The two main methods for classification used in this work were manual (Section 6.1) and algorithmically-based (Section 6.2). Since classification methods rely on a set of parameters to characterize an object, a manual classification system was felt to yield a better system since it is difficult to develop classifiers that encompassed prior knowledge, experience, and intuition. However, this method is very subjective and may be difficult for more complex objects, which may create ambiguities when determining how to classify an object. An algorithmically-based method would be more objective but requires time and effort to develop.

\subsection{Manual Classification}

Three approaches were considered for the manual classifications. These approaches are described in the following sub-sections.

\subsubsection{Geometric Approach}

This approach involves classifying objects based on their geometry where similar shaped objects were put into the same class. The objects were grouped based on viewing a 2D image of the object and other information regarding scale or size was not considered. The obvious drawback to classifying objects based on geometry is that many objects are a combination of various basic geometric shapes and judgment will have to be depended upon.

The objects from the database were placed into six classes, A to F, and the groupings are given in Table 6.1. In general, the objects in Classes A to F consisted of:

- Class A - flat and thin (high aspect ratio) objects; objects that were "2D" or planar

- Class B - gears, wheels, or round objects

- Class C - bulky or block-like objects

- Class D - rounded/curved objects that did not fit into Class B

- Class E - objects made with flat, thin material; objects similar to Class A but that are "3D" (e.g., bracket). Objects in this class are also more angular than those in Class D.

- Class F - cylindrical or tubular type objects

Once the classification was performed, bar plots of each metric (surface area, volume, etc.) were generated. Two of these plots are shown in Figure 6.1. The plots were made to determine if there was any clustering of the metrics for a given class. For example, from visual inspection, two general observations based on Figure 6.1 are that, on average, objects in Class $C$ have higher volumes, and that the compactness (defined as the ratio of maximum extent to minimum extent of an object) for objects in Class A were higher than those in the other classes. These observations are noted in Table 6.1. An observation of High/Low was made only if it was clearly evident in the plot; if there was no clear evidence, no observation of High/Low was noted for the metric (see Figure 6.2). As this is a visual observation, the determination of High/Low is subjective. These plots were generated for all classifications - manual and algorithmic based. 


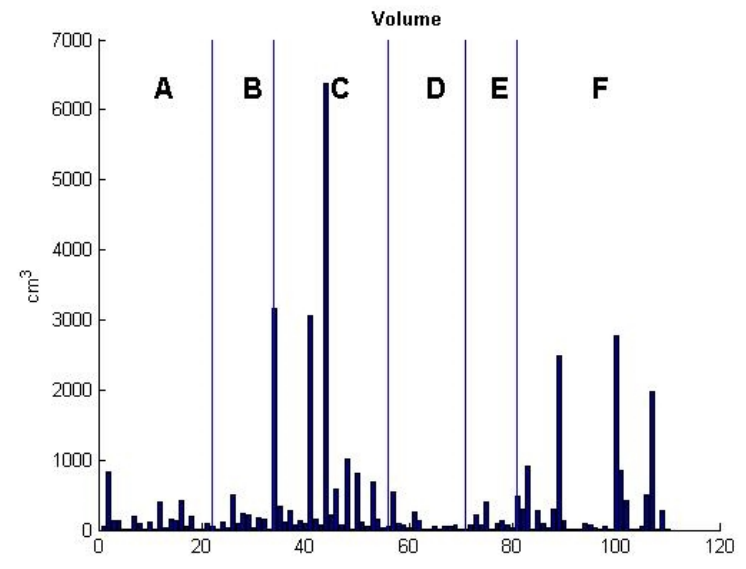

a. Volume

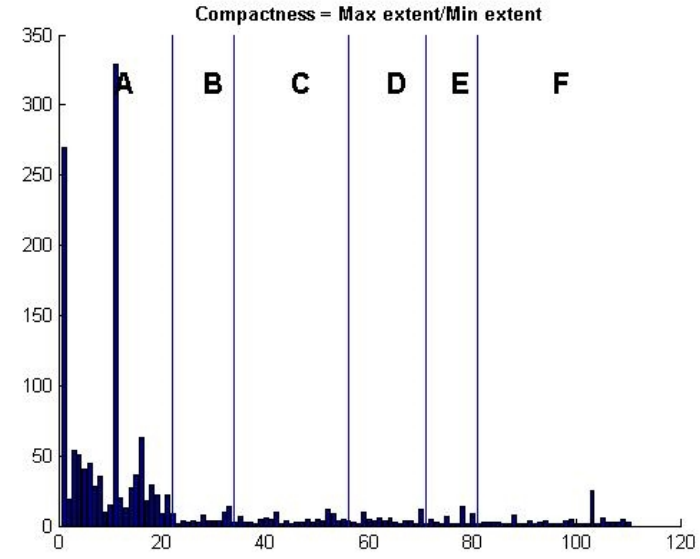

b. Compactness: Max extent/Min extent

Figure 6.1. Plots of (a) Volume and (b) Compactness for objects in classes A to F.

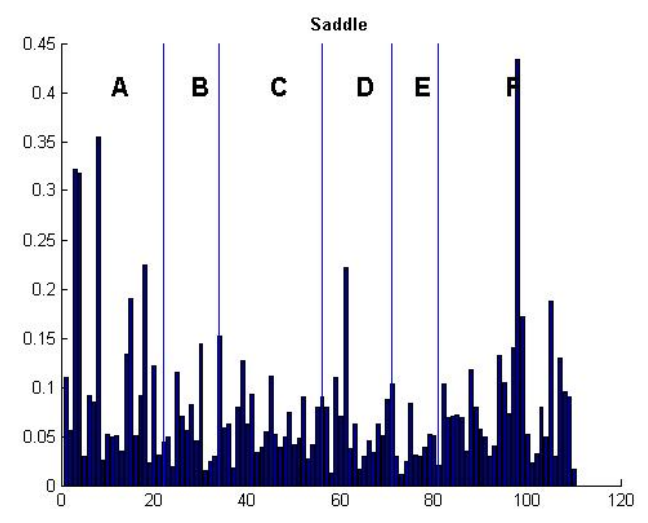

Figure 6.2. Plot of the Saddle metric shows no clear indication of High/Low for a given class.

An objective way to determine whether a metric was High/Low for a given class would be based on the comparison of the statistical averages for each class for a given metric. However, the standard deviations of the average for many of the classes are large (in some cases extremely large) indicating that the observations based on the average would not be statistically significant. Thus, this method was not used. 
Table 6.1. Classification based on object geometry.

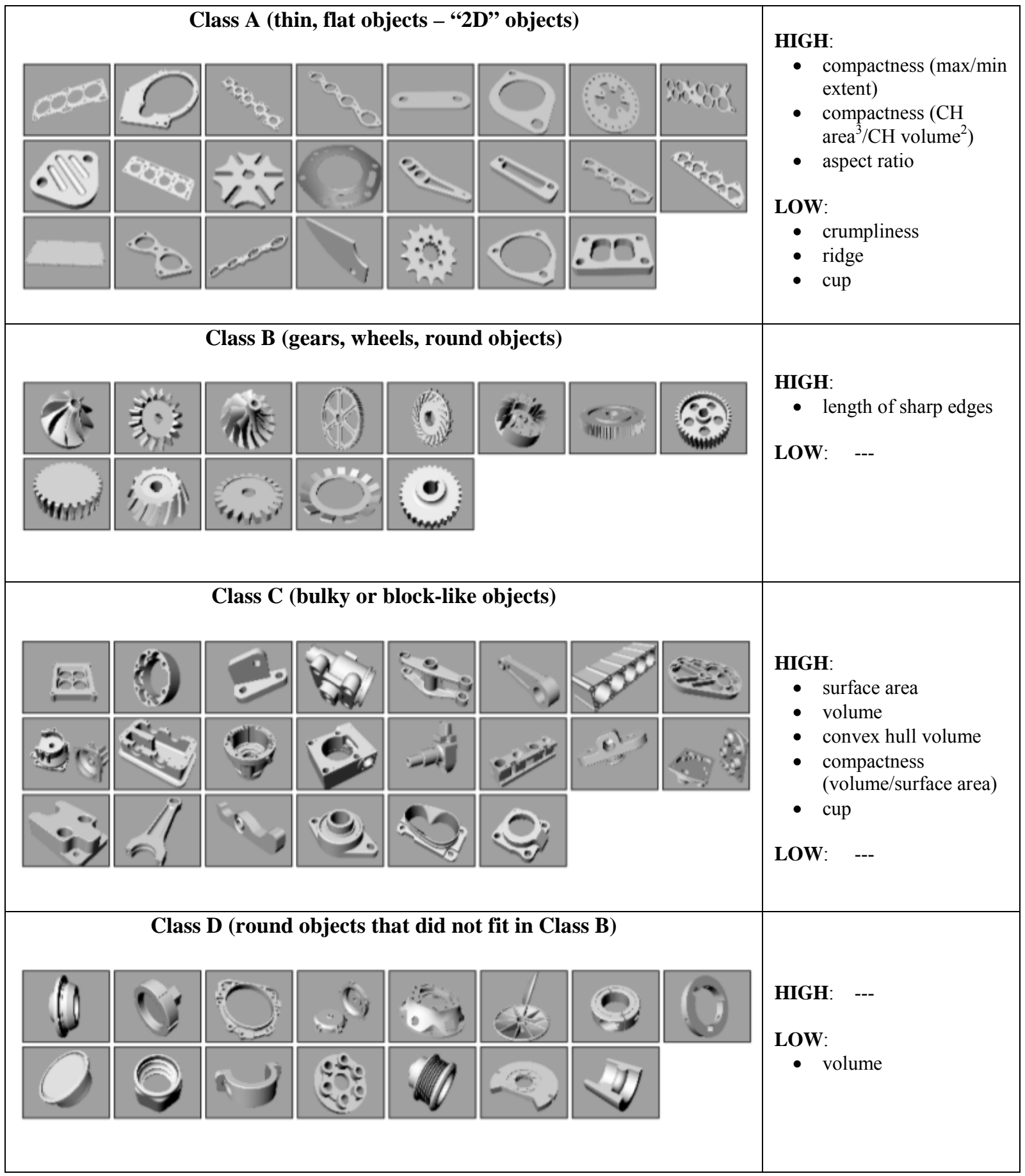




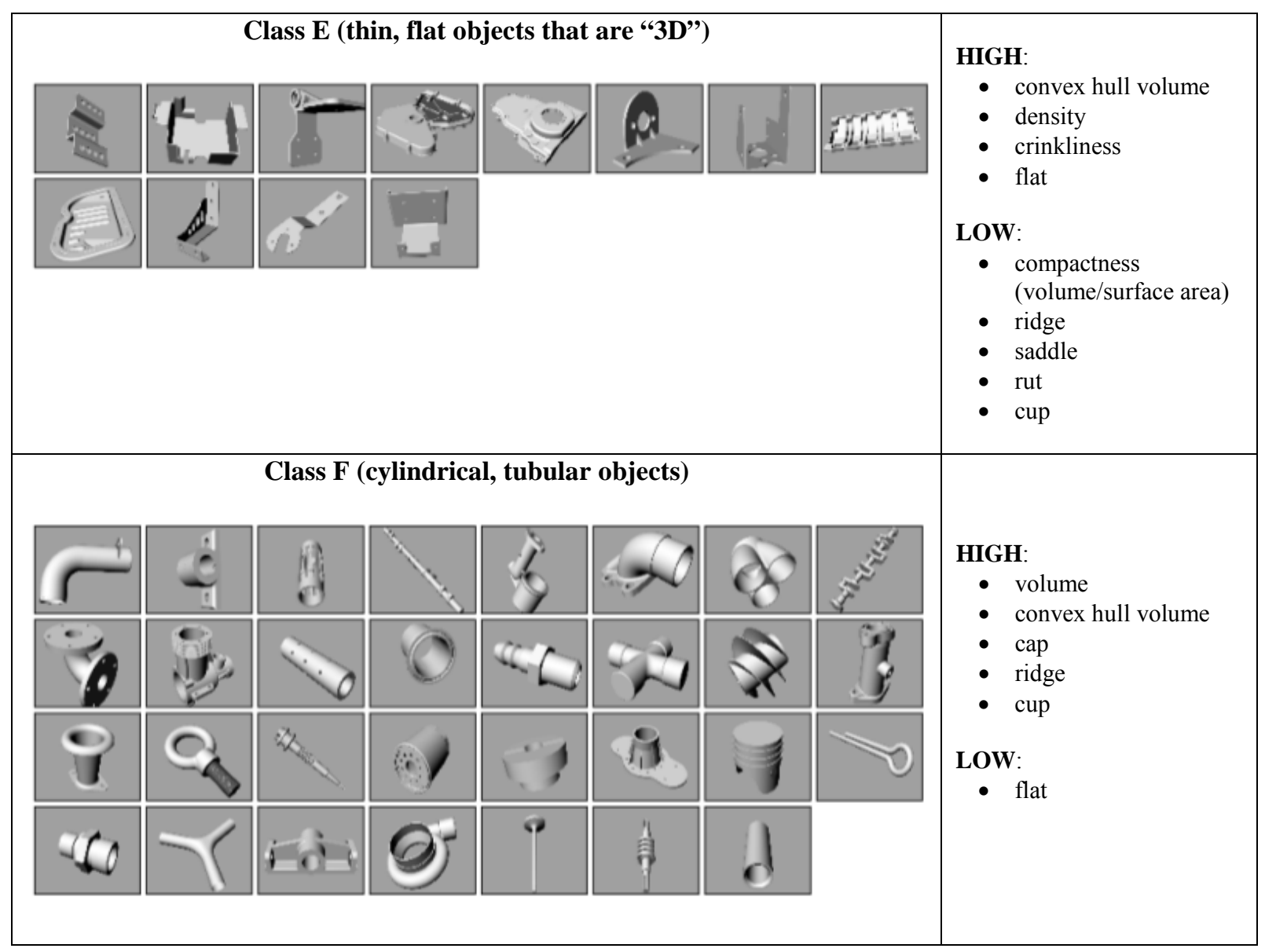

\subsubsection{Laser-based Technology Approach}

This approach grouped the objects in the database based on how difficult it would be for a particular type of perception system to obtain the pose of the object given certain conditions. The objects were placed into one of five levels (i.e., 5 classes) of difficulty as follows:

\section{Levels of Difficulty}

1 - Very Easy: All 6 DOF (degree of freedom; 3 positional and 3 rotational) can be obtained with relatively high accuracy

2 - Easy: All DOF can be obtained, but with relatively low accuracy

3 - Medium: Only 4 or 5 of the DOF can be obtained with relatively high accuracy

4 - Hard: Only 4 or 5 of the DOF can be obtained, but with relatively low accuracy

5 - Very Hard: Only the 3 positional DOF can be obtained

In order to aid in gauging the difficulty of measuring an object's pose using a certain perception system, three additional metrics were defined for an object: 1) Pose Unambiguity, 2) DOF Needed, and 3) Ambiguous DOF Needed. Definitions for these metrics and possible values for them are presented below. 
Pose Unambiguity: Measure of how many degrees of freedom can be determined unambiguously for an object when all relevant features are visible.

3 - Only a 3DOF position can be calculated unambiguously

4 - Only a 4DOF pose can be calculated unambiguously

5 - Only a 5DOF pose can be calculated unambiguously

6 - No ambiguity, full 6DOF pose can be obtained unambiguously

DOF Needed: Number of degrees of freedom that must be calculated in order to properly assemble the object into a larger assembly. Although in some cases, some of the degrees of freedom may remain ambiguous (e.g., differentiating between the front and back face of a flat washer) that degree of freedom may still need to be calculated in order to assemble the object (e.g., an assumption must be made about which is the front side of a flat washer in order to calculate its two angles of rotation which will allow a robot to insert a screw through it).

3 - Only a 3DOF position must be calculated (e.g., ball bearing)

$4-4$ DOF must be calculated

5 - 5DOF must be calculated

6 - All 6DOF must be calculated

Ambiguous DOF Needed: Number of ambiguously-defined DOF that need to be calculated. This is the difference between DOF Needed and Pose Unambiguity.

For each object in the database, values for the above three metrics were first assigned.

\section{Perception Systems}

For this approach, the perception systems for which the object pose determination difficulty would be gauged had to be defined. To do this, a perception system was defined as a combination of four factors: 1) technology, 2) clutter, 3) background, and 4) presentation. Definitions for these four factors and possible values for them are explained below.

Technology: Sensor hardware is based on:

1) a laser line scanner mounted on a $6 \mathrm{DOF}$ arm,

2) a multiple camera machine vision system in which one or more of the cameras are mounted on a $6 \mathrm{DOF}$ arm,

3) a structured light area scanner mounted on a 6DOF arm, or

4) a combination of laser line scanner, structured light area scanner and machine vision in which the object is manipulated with a gripper in order to present the object more favorably to one or more of the sensors.

Clutter: Object is 1) isolated or 2) there are other identical objects in the field-of-view of the sensor(s).

Background: Object is 1) on a flat surface or 2) in a bin.

Presentation: Object is presented to the sensor(s) 1) in a favorable orientation or 2) with important features occluded. 
Using the above factors, a perception system may be described using four numbers. For example, System 1111 means a perception system using a laser line scanner to determine the pose of an isolated object on a flat surface presented in a favorable orientation. Similarly, a System 1221 means a perception system using a laser line scanner to determine the pose of an object in a bin with other identical objects, and presented in a favorable orientation (e.g., at the top of the pile anywhere in the bin with relevant features visible).

\section{Assumptions}

In order to gauge the difficulty of a certain perception system's ability to determine the pose of an object, several assumptions regarding the object, its environment, and the perception system itself were made. These assumptions are presented below.

1. The object is stationary.

2. The object material, color, and texture are favorable to the technology being used.

3. Lighting conditions are favorable to the technology being used.

4. The environment is stable throughout the perception task.

5. Perception systems that are based on machine vision technologies have a higher resolution than those that are based on laser line scanning technologies and therefore, can more accurately measure the poses of smaller objects.

6. Perception systems that are based on laser scanning technologies can more accurately measure the pose of volumetric objects (i.e., objects with features in all three dimensions as opposed to flat objects) than perception systems that are based on machine vision technologies.

7. Perception systems that use structured light area scanning technologies or a combination of laser line scanning and machine vision inherit the best characteristics of the laser line scanning- and machine vision-based perception systems.

8. All the perception systems use optimized algorithms (e.g., data processing, filtering, fitting, etc.) in order to calculate the poses of objects using the raw sensor data.

9. Perception systems that use laser line scanning and structured light area scanning technologies yield more accurate poses of objects that are presented among many other identical objects (i.e., cluttered background) because it is easier to isolate (segment) the data from a single object among many identical objects using $3 \mathrm{D}$ data.

Placing the object in a bin with other identical parts and in a favorable orientation adds one level of difficulty to each kind of perception system used with the same object isolated on a flat surface.

Theoretically, the above method can result in many possible combinations of factors, which could define a large number of perception systems. For the purposes of this classification, only six different systems (1111, 1221, 2111, 2221, 3111, and 3221) were considered. For Presentation, only the case of favorable orientation (represented by the fourth digit in the nomenclature with a value $=1$ ) was used as the alternative of 2 (important features occluded) would yield numerous classifications depending on which features were occluded.

The classification for Systems 1111, 2111, and 3111 are given in Tables 6.2 to 6.4. Systems 1221, 2221, and 3221 are not shown as they are similar to Systems 1111, 2111, and 3111, respectively; the only change being the difficulty levels for 1221, 2221, and 3221 were increased by 1 (i.e., the classes increased 
by 1). No difficultly level was higher than Class 5 - Very difficult. For example, for System 1111, two objects were placed in Class 5. For System 1221, this meant that the difficulty level would have been Class 6, but since Class 5 was the highest level, these two objects in Class 5 for System 1111 were placed into Class 5 for System 1221.

As mentioned earlier, High/Low observations were made based on bar plots of each of the metrics. However, in a couple of classes (System 1111 - Class 5 and System 3111 - Class 3), there were only two objects in that class. Therefore, the addition of one or more objects could potentially reverse or nullify the High/Low observations, i.e., these observations have a low level of confidence associated with them. 
Table 6.2. Classification for System 1111: Technology = laser line scanner mounted on 6DOF arm, Clutter $=$ isolated, Backround $=$ on flat surface, Presentation $=$ favorable orientation .

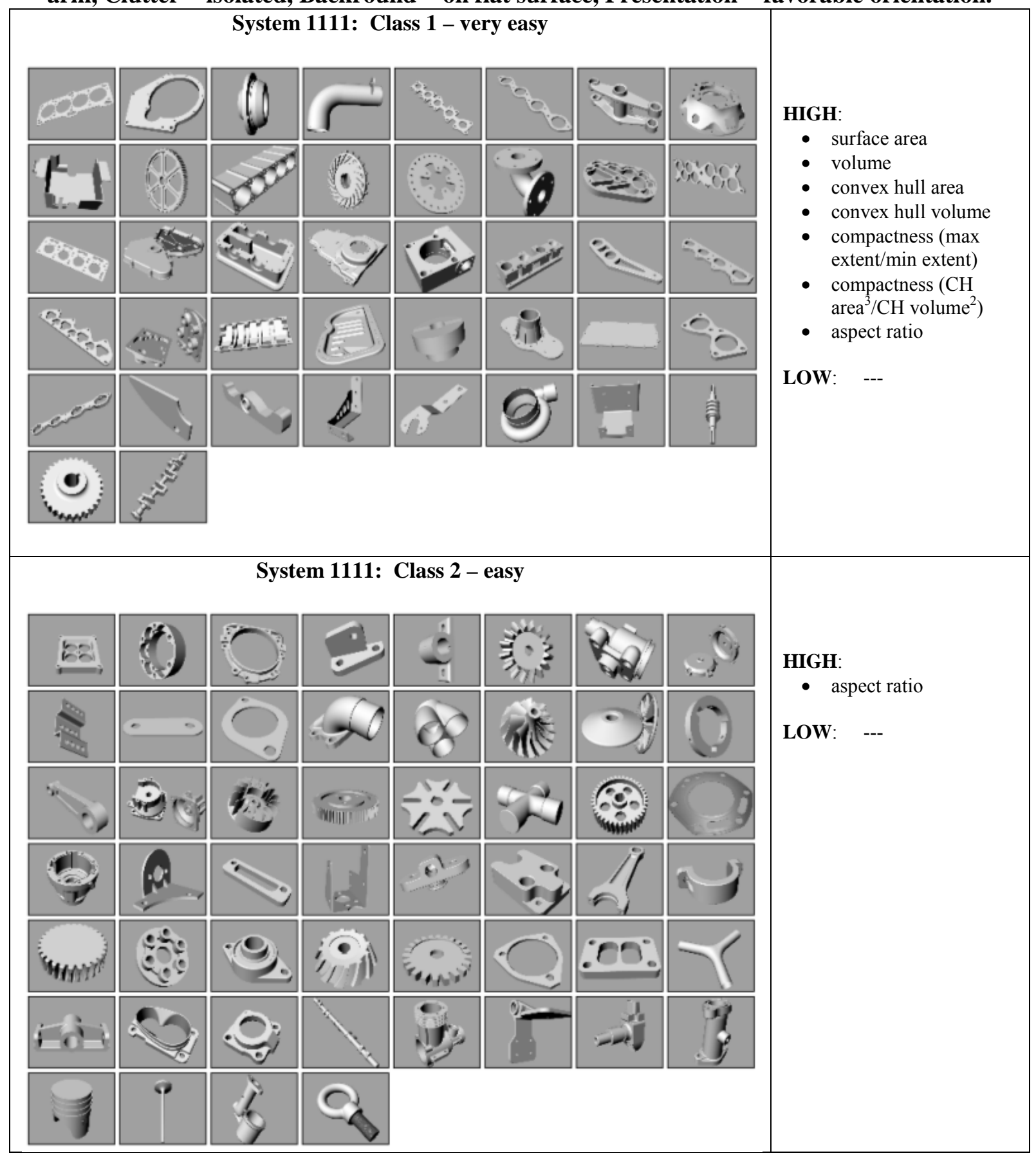




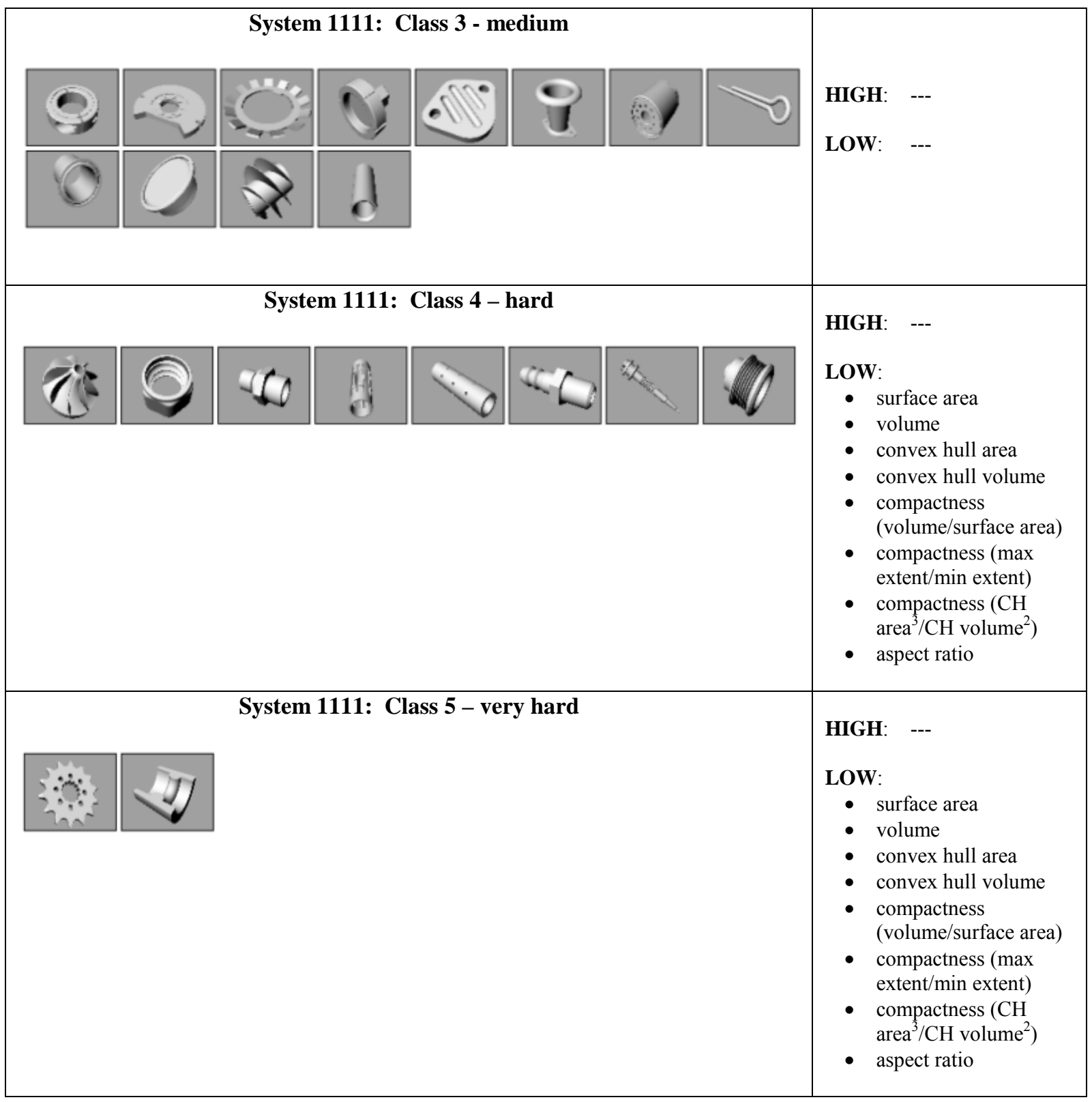


Table 6.3. Classification for System 2111: Technology = multiple camera machine vision system in which one or more of the cameras are mounted on a 6DOF arm, Clutter = isolated, Backround = flat surface, Presentation = favorable orientation.

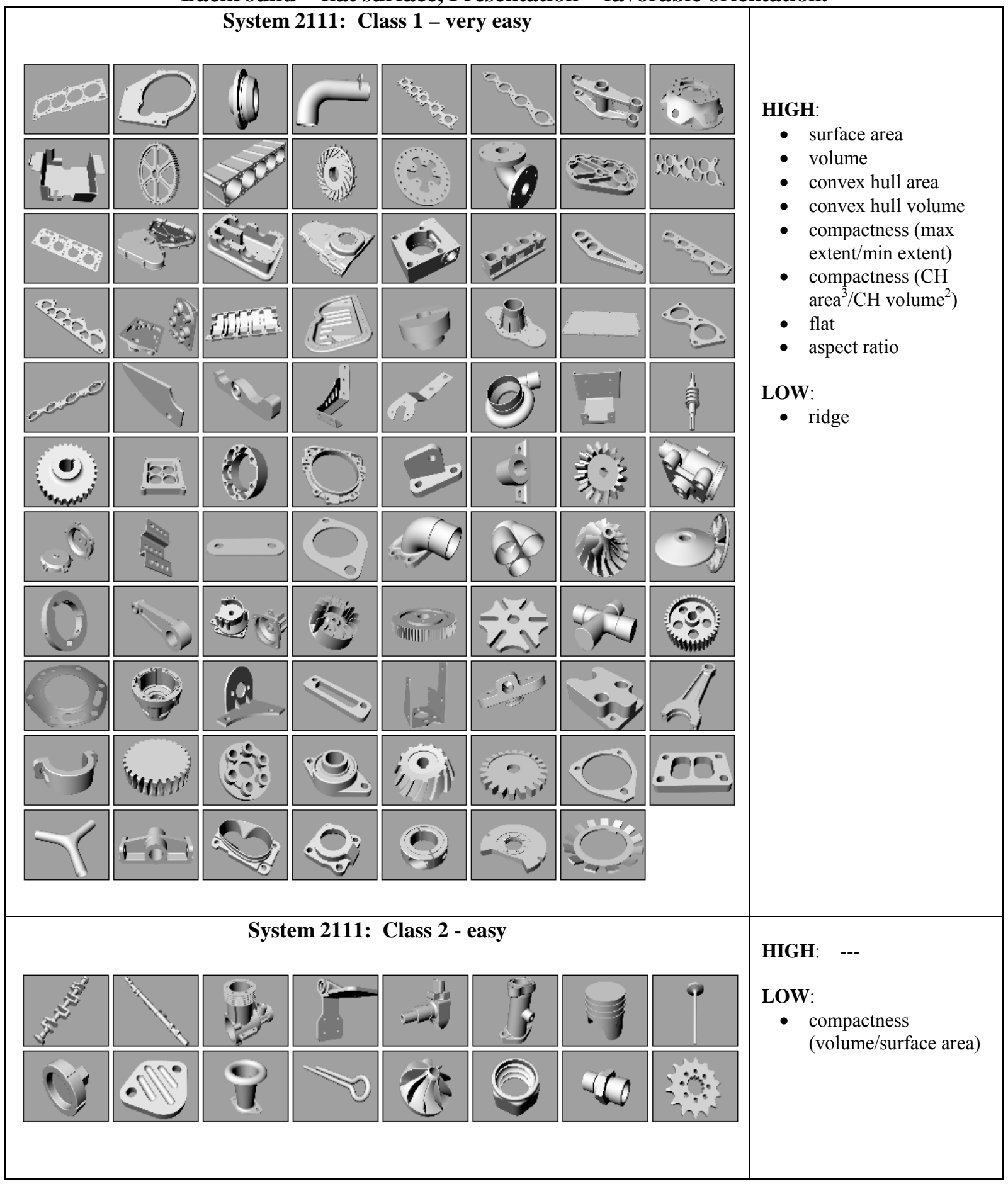




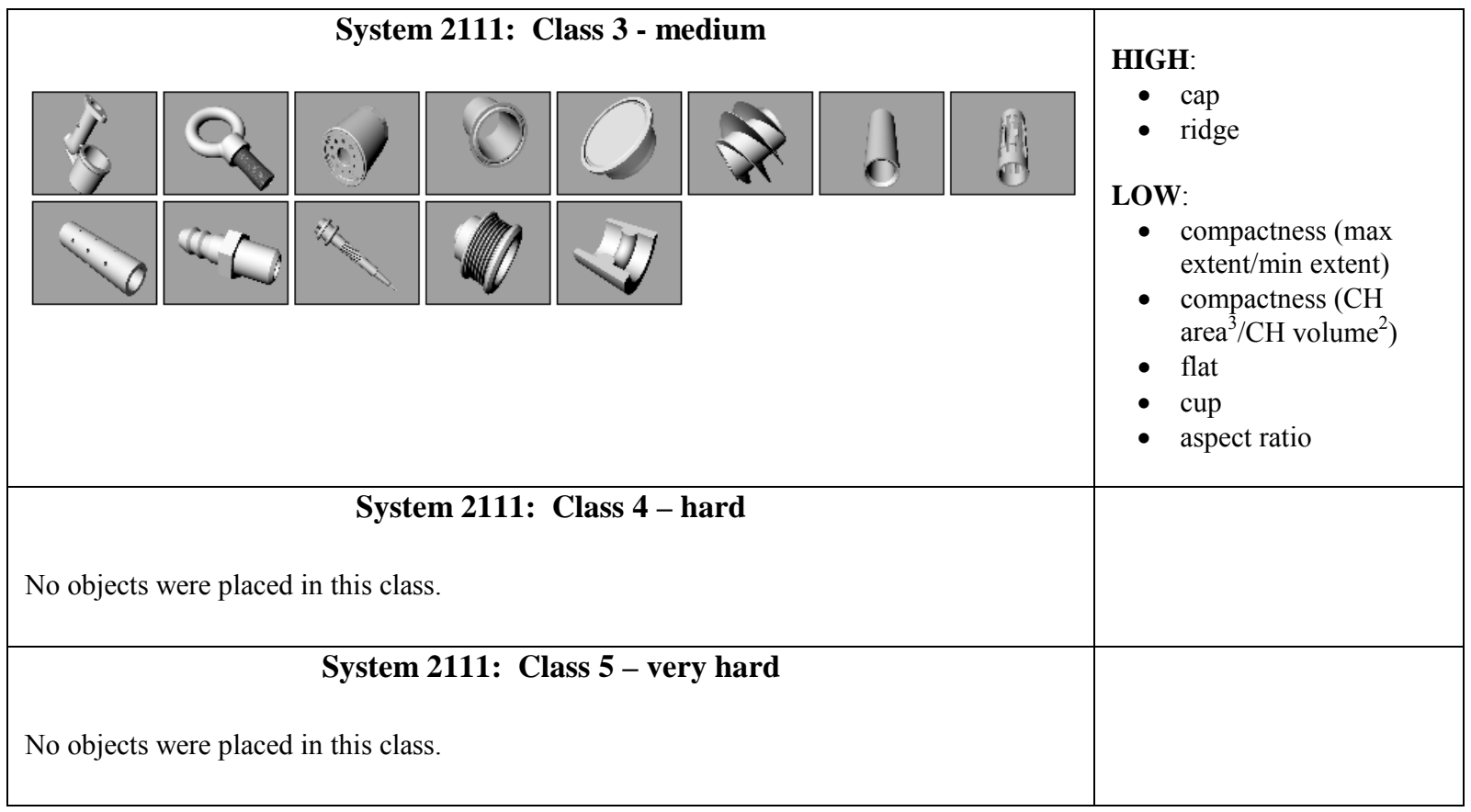


Table 6.4. Classification for System 3111: Technology = structured light area scanner mounted on a 6DOF arm, Clutter = isolated, Backround = flat surface, Presentation = favorable orientation.

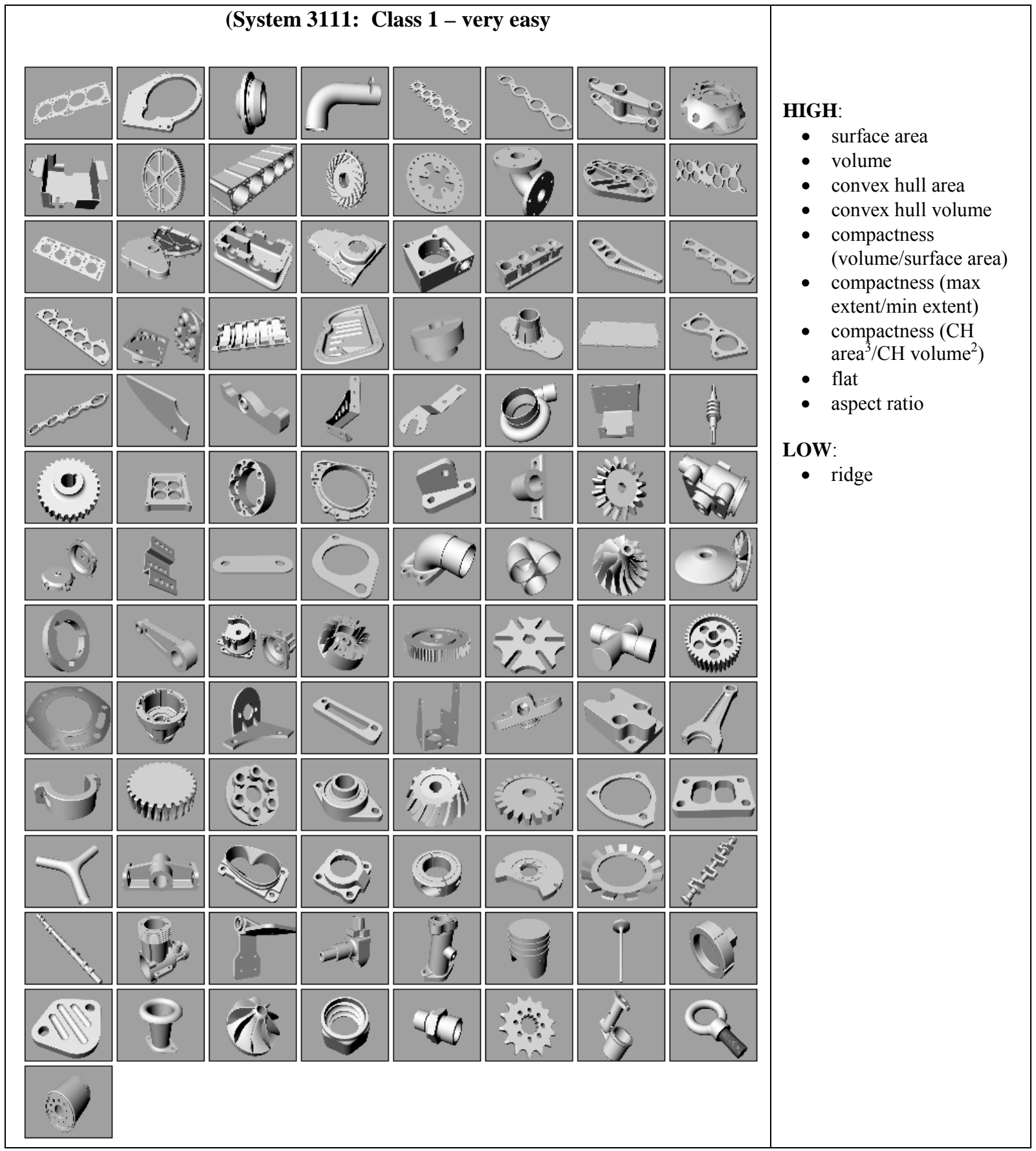




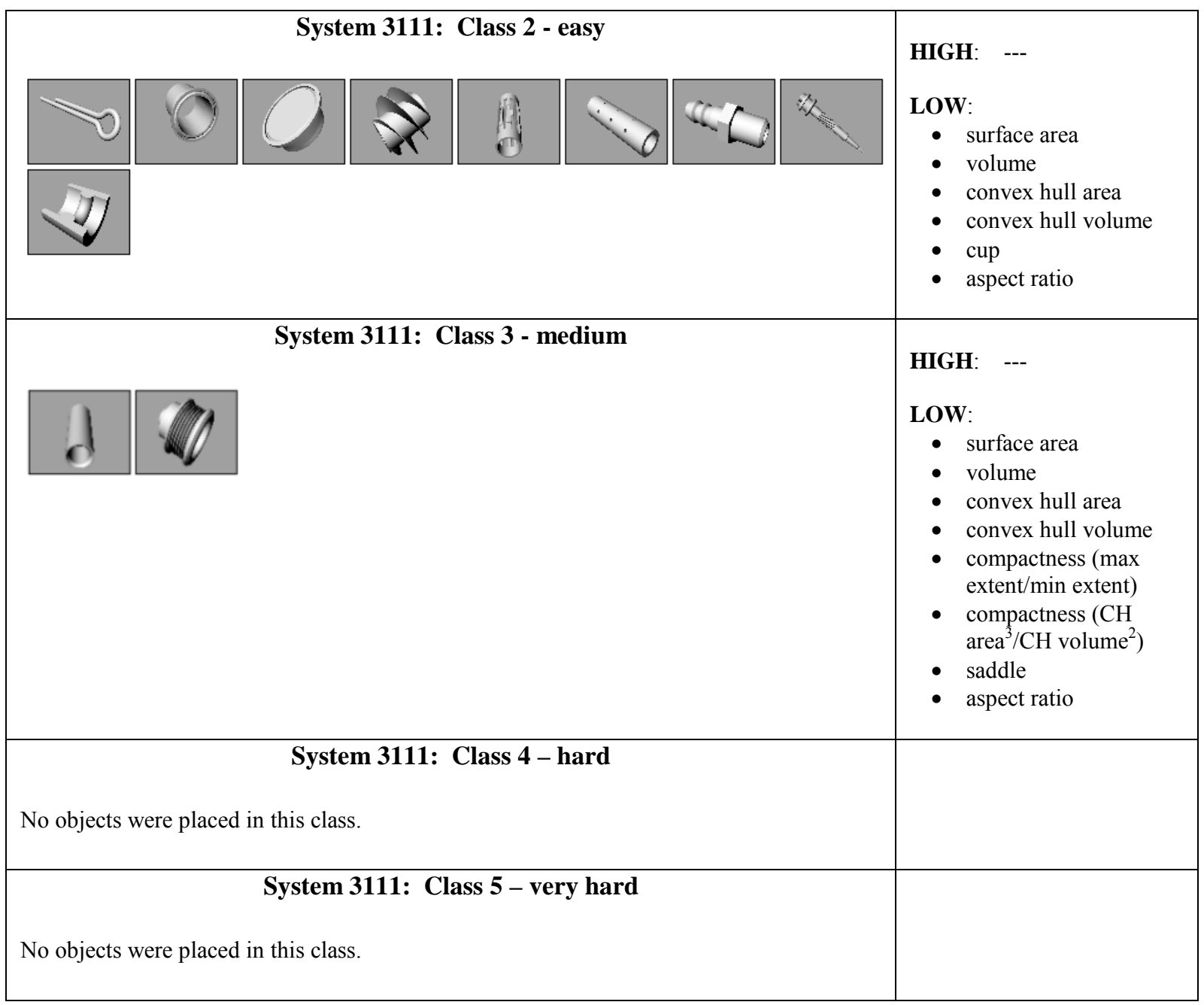

\subsubsection{Vision-based Technology Approach}

This approach attempts to classify the objects for vision-based perception systems using the following criteria for obtaining pose in the openCV (Bradski and Kaeber, 2008) and the Point Cloud Libraries (Rusu, 2011):

1. Quality and quantity of an object's perception features

2. Complexities of algorithms to compute those features

3. Uniqueness of those features from different perceptive views

Based on the above criteria, each object was placed into 10 levels. The criteria for the pose score are:

- Score $1 \&$ 2: If all of the following are true:

0 the object has no unique features in all views;

0 it is difficult to obtain visual features of the object (e.g., small holes in the object);

0 the object features are quadratic or higher order.

- Score $3 \& 4$ : if all of the following are true: 
o the object has at least one medium unique feature (e.g., feature cannot be easily detected) in one to two views;

o one to two linear features (e.g., line, edge, point, corner).

- Score $5 \&$ 6: if all of the following are true:

0 the object has at least one good unique feature (e.g., feature can easily be detected) in three to four views;

0 three to four linear features.

- Score $7 \&$ 8: if all of the following are true:

0 the object has at least one excellent unique feature (e.g., feature is very easily detected) in five to six views;

0 five to six linear features.

- Score 9 \& 10: if all of the following are true:

0 the object has several excellent unique features in all views;

0 many linear features.

The following procedure to classify the 116 objects into 10 classes:

1. Load the CAD file of each object into MeshLab software

2. View the object from the top, bottom, left, right, front, and back. From these views, the quantity and quality of the features as well as their uniqueness were determined.

3. Based on Step 2, assign a pose score to the object.

4. Group objects with the same pose score into a class: Pose score $1=$ Class 1 , etc.

For objects in Classes 1 to 4, it would be very problematic for the perception systems to determine their poses. Perception systems would have very little problems in accurately measuring the poses of objects in Classes 7 to 10. 
Table 6.5. Vision-based Perception System Classification Approach. Class $1=$ very difficult, Class 10 = very easy.

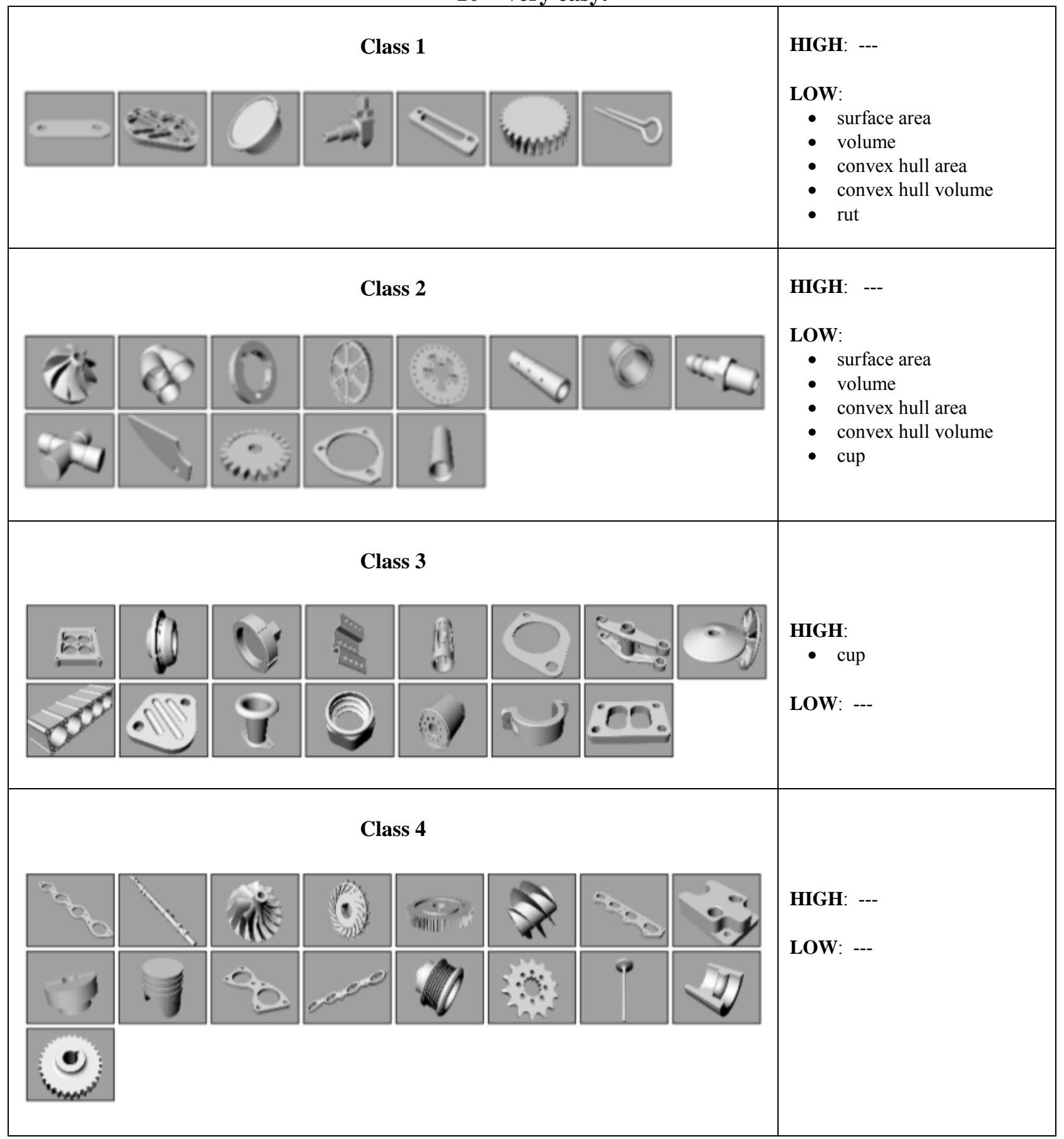




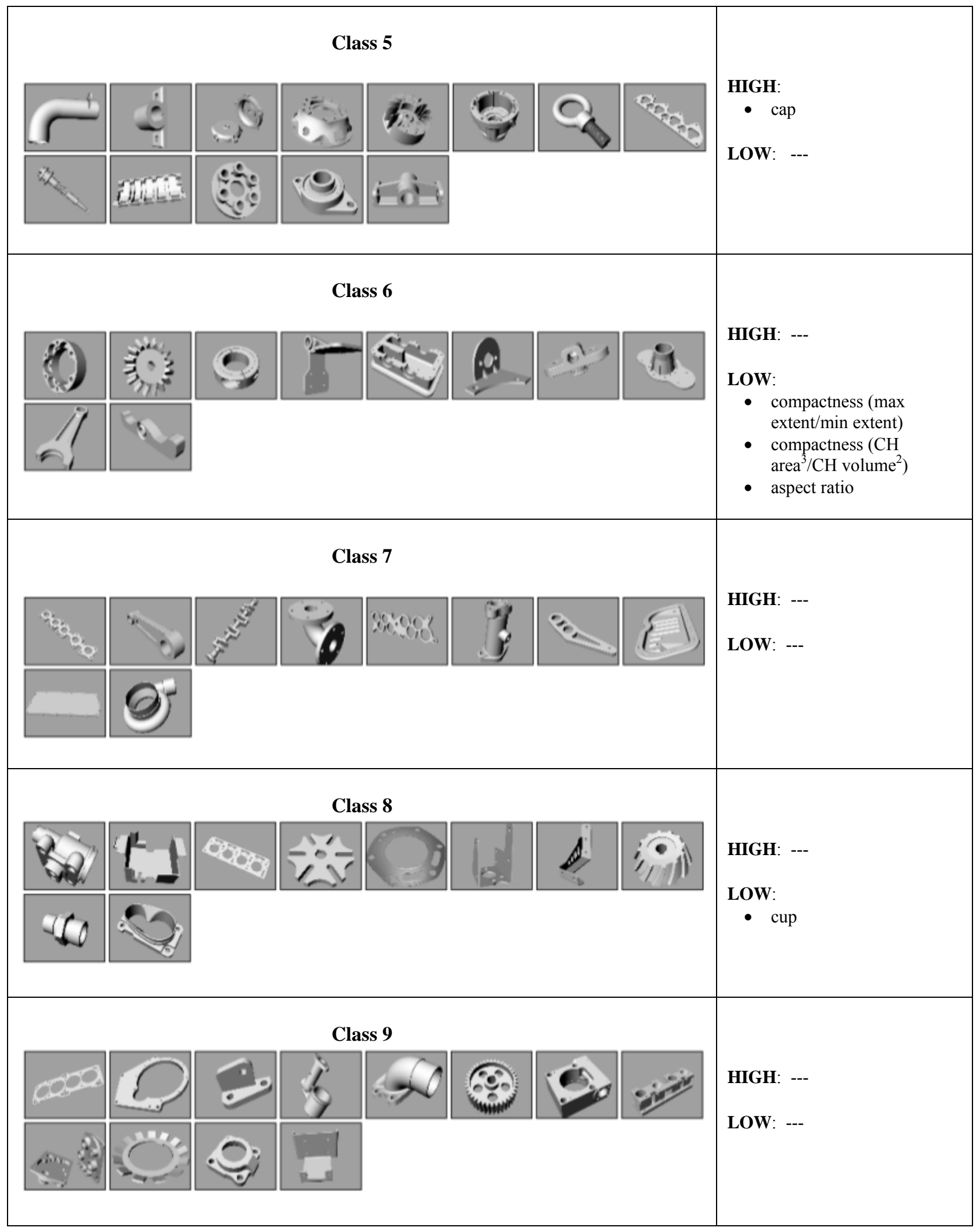




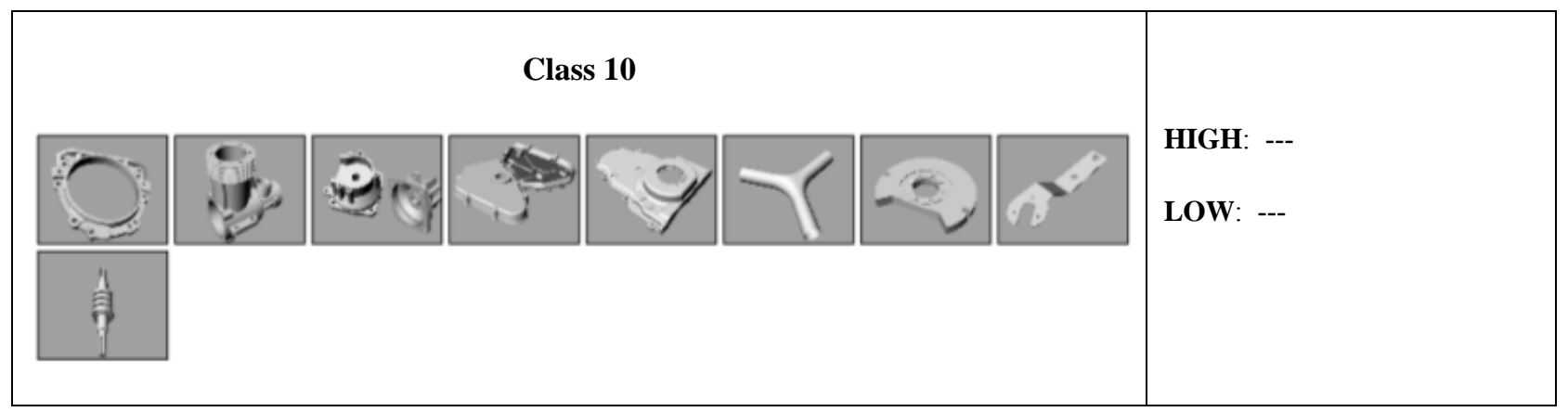

\subsection{Classification based on Clustering}

We used the metrics given in Table B1 for clustering. Clustering, in this work, is the process of organizing a set of 3D CAD models into groups in such a way that the $3 \mathrm{D}$ models within the group are more similar to each other than they are to other objects belonging to different groups. Clustering is also known as an unsupervised learning technique in the scientific literature. We use $k$-means cluster analysis (Teknomo and Kardi, 2006) along with the shape metrics and a user-defined number of clusters to classify the 3D CAD models. We assume that the models as represented by the shape metrics would allow us to distinguish one group from another.

The $k$-means algorithm is an algorithm to cluster $n$ objects based on attributes into $k$ partitions, $k<n$. It is similar to the expectation-maximization algorithm for mixtures of Gaussians in that they both attempt to find the centers of natural clusters in the data, where there are $k$ clusters. In this work, we used $k=5, k=$ 6 , and $k=10$ as these were the number of classes used for the manual classifications. The results of the clustering based on $k=5, k=6$, and $k=10$ are shown in Table 6.6, Table, 6.7, and Table 6.8, respectively.

The metrics we used for $k$-means clustering are only based on shape type (see Section 5, Table 5.1). The other metrics were not used since there is a large variation in the size of the different models and most of the other shape metrics are scale dependent. 
Table 6.6. $k$-means clustering with $k=5$ using the shape index metrics.

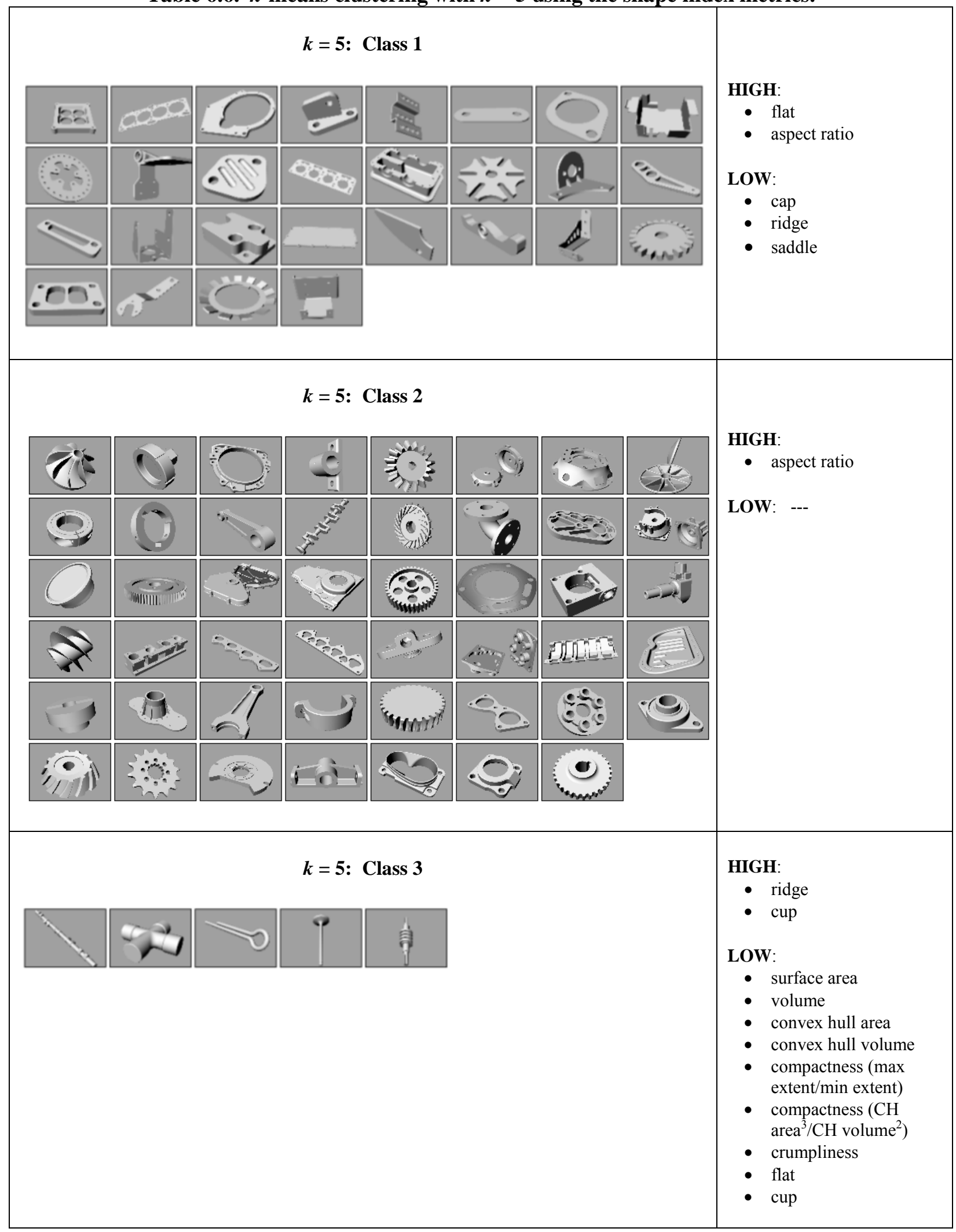




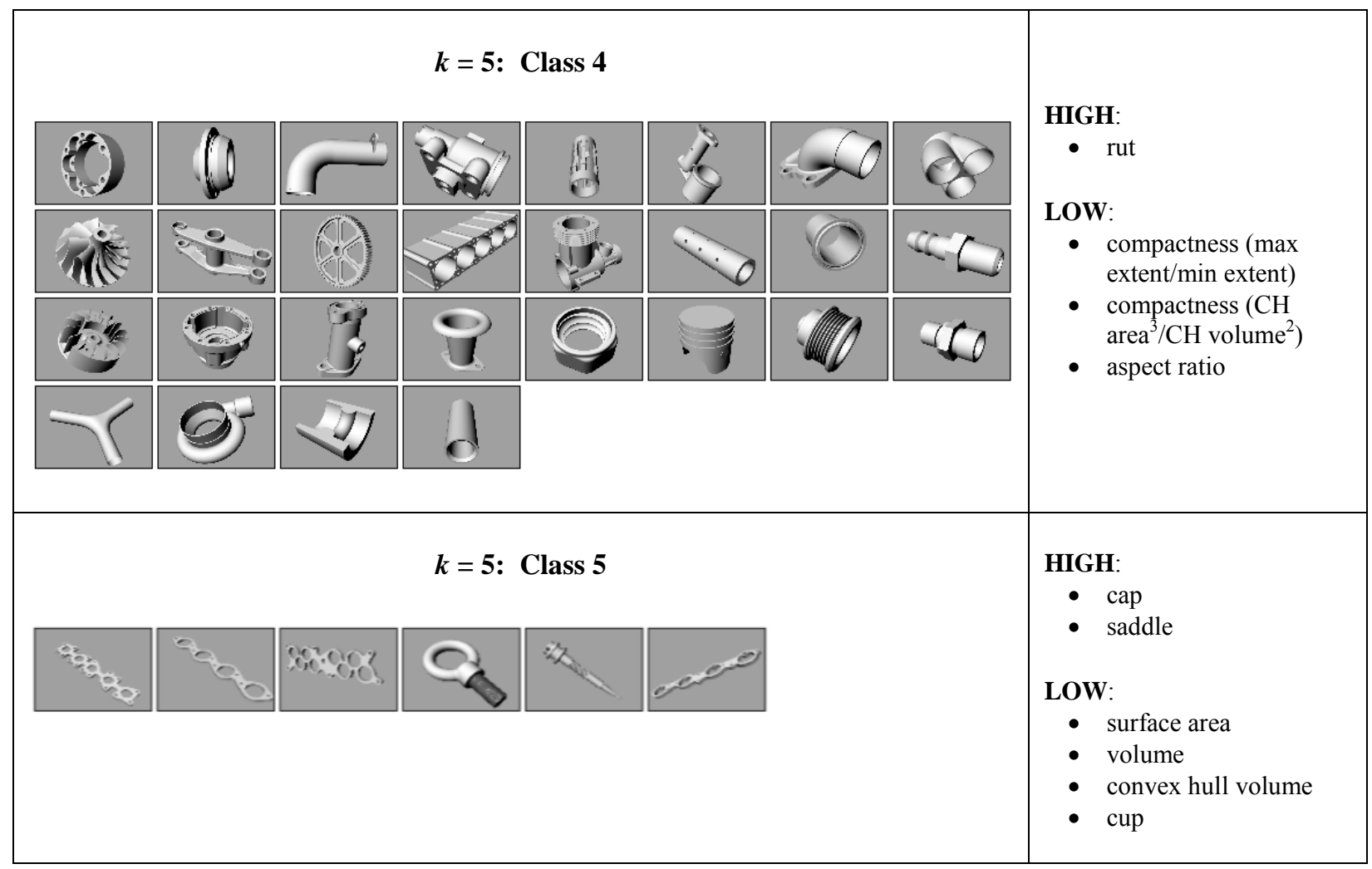


Table 6.7. $k$-means clustering, $k=6$ using the shape index metrics.

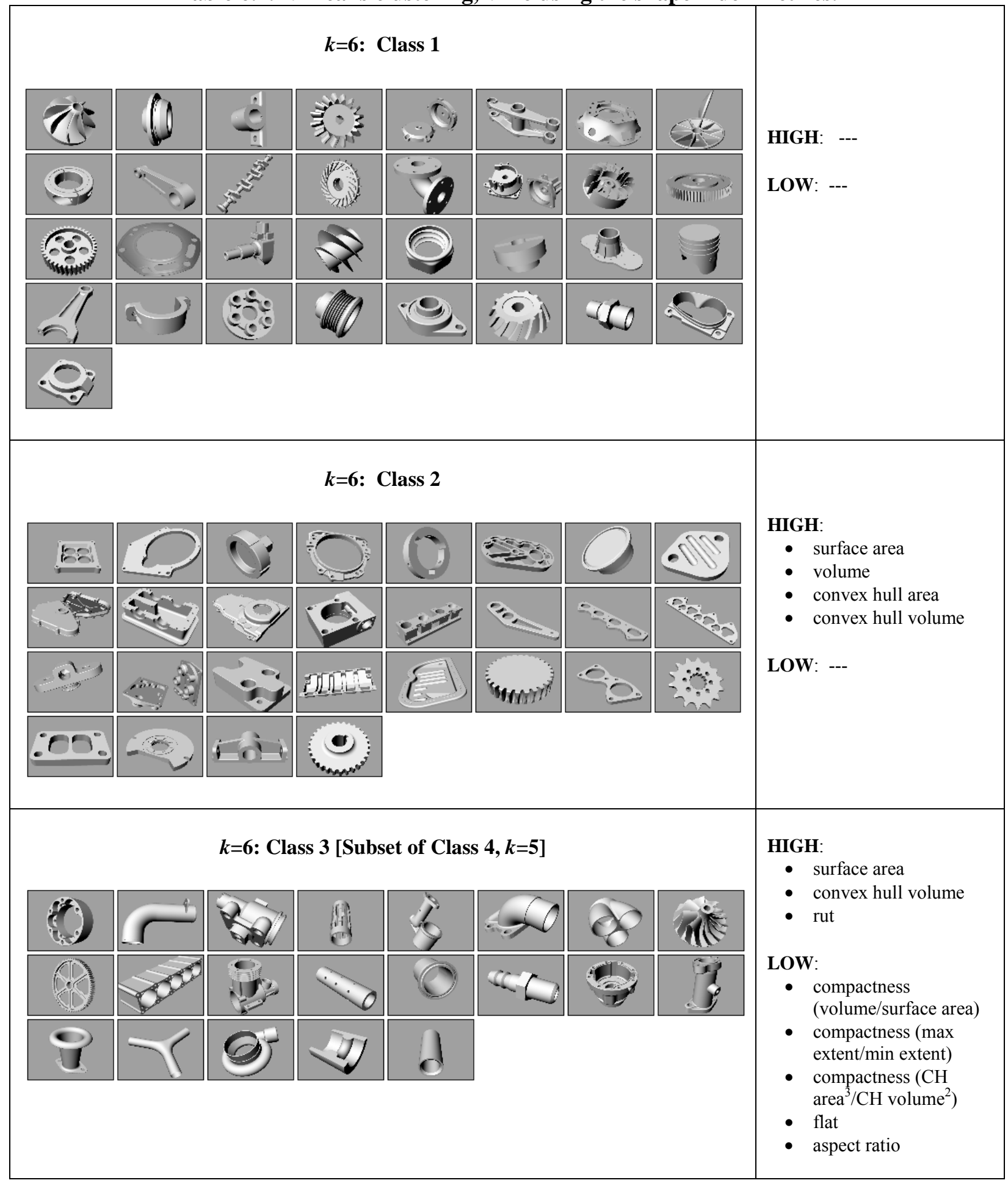




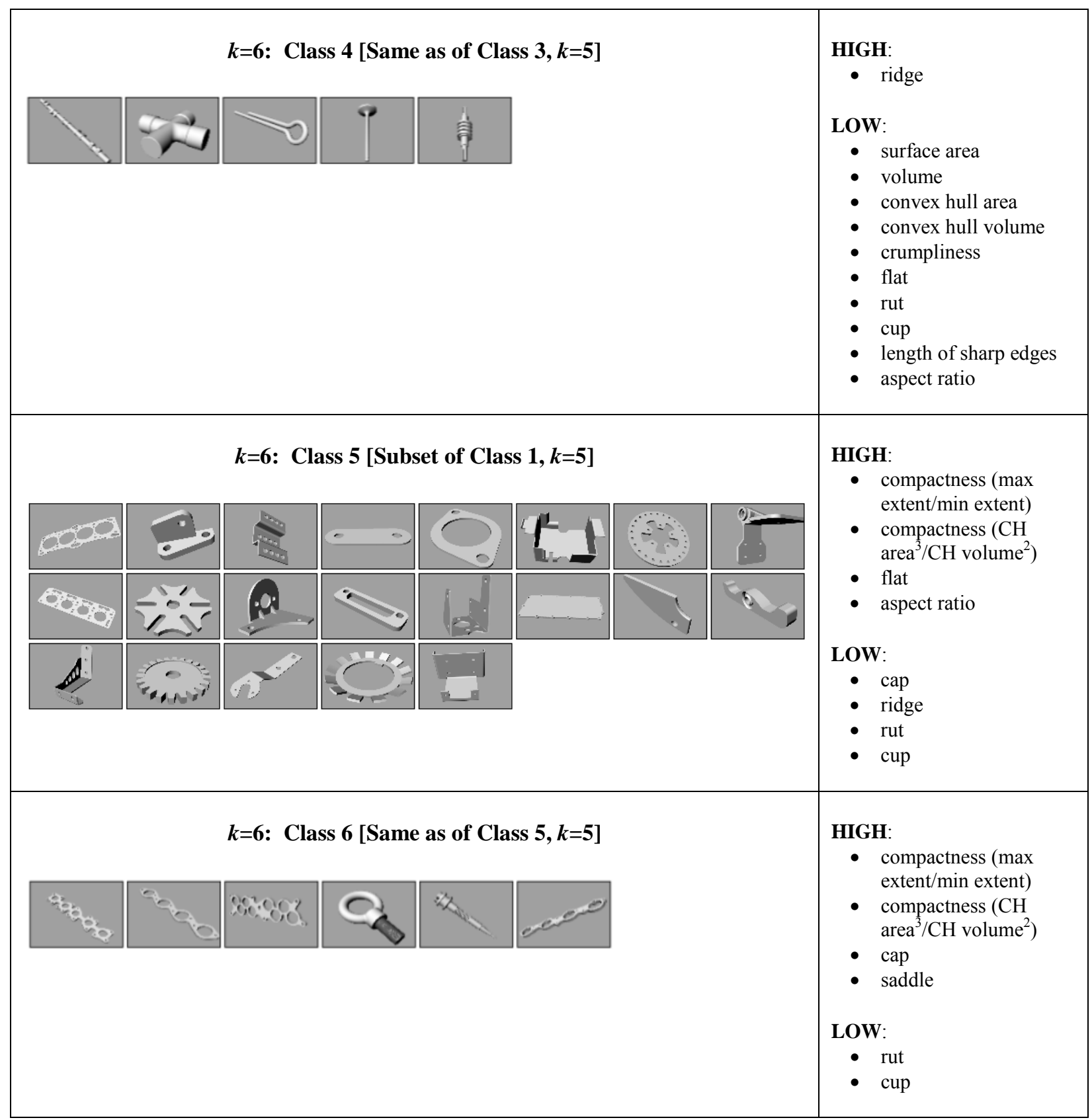


Table 6.8. $k$-means clustering, $k=10$ using the shape index metrics.

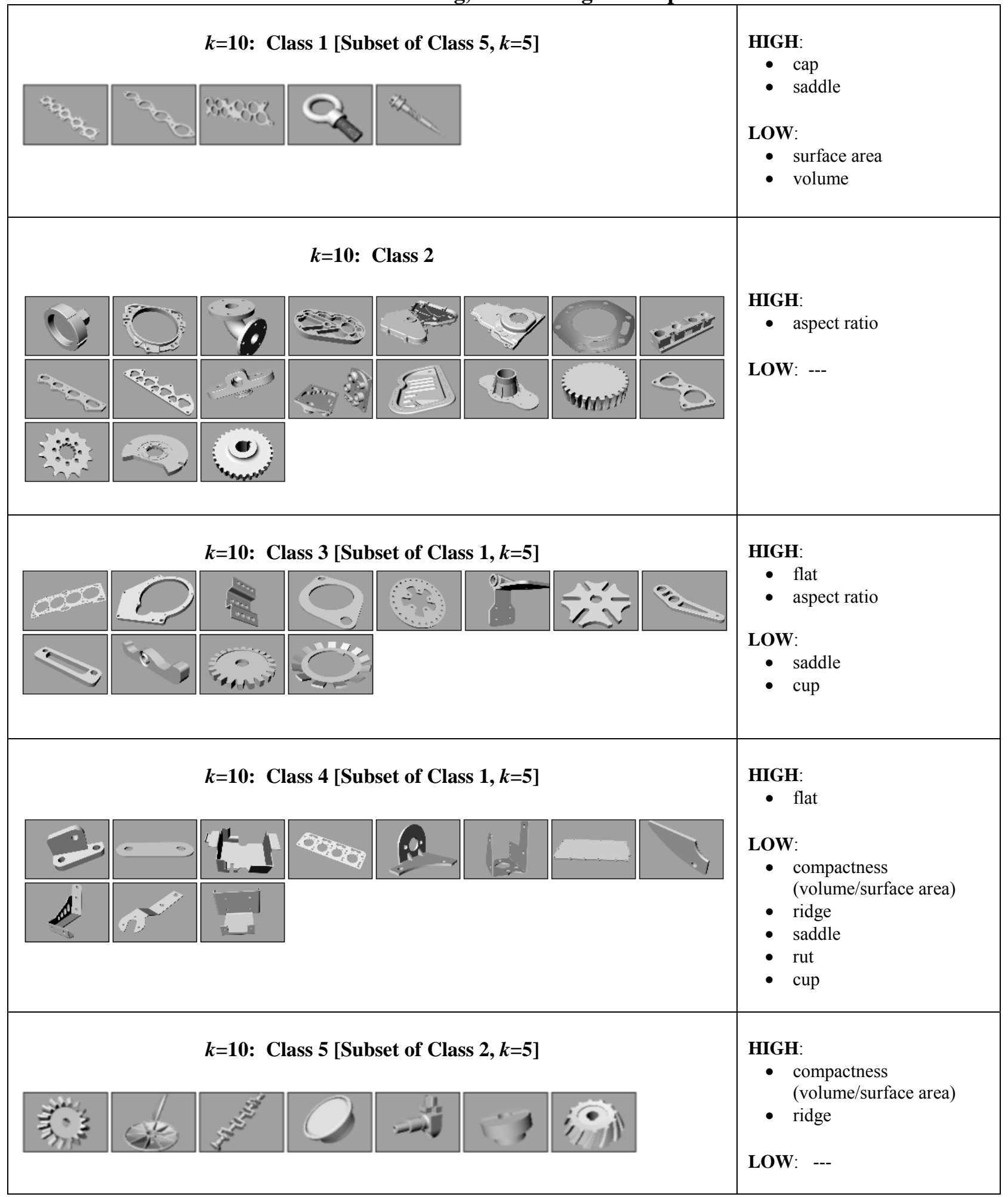




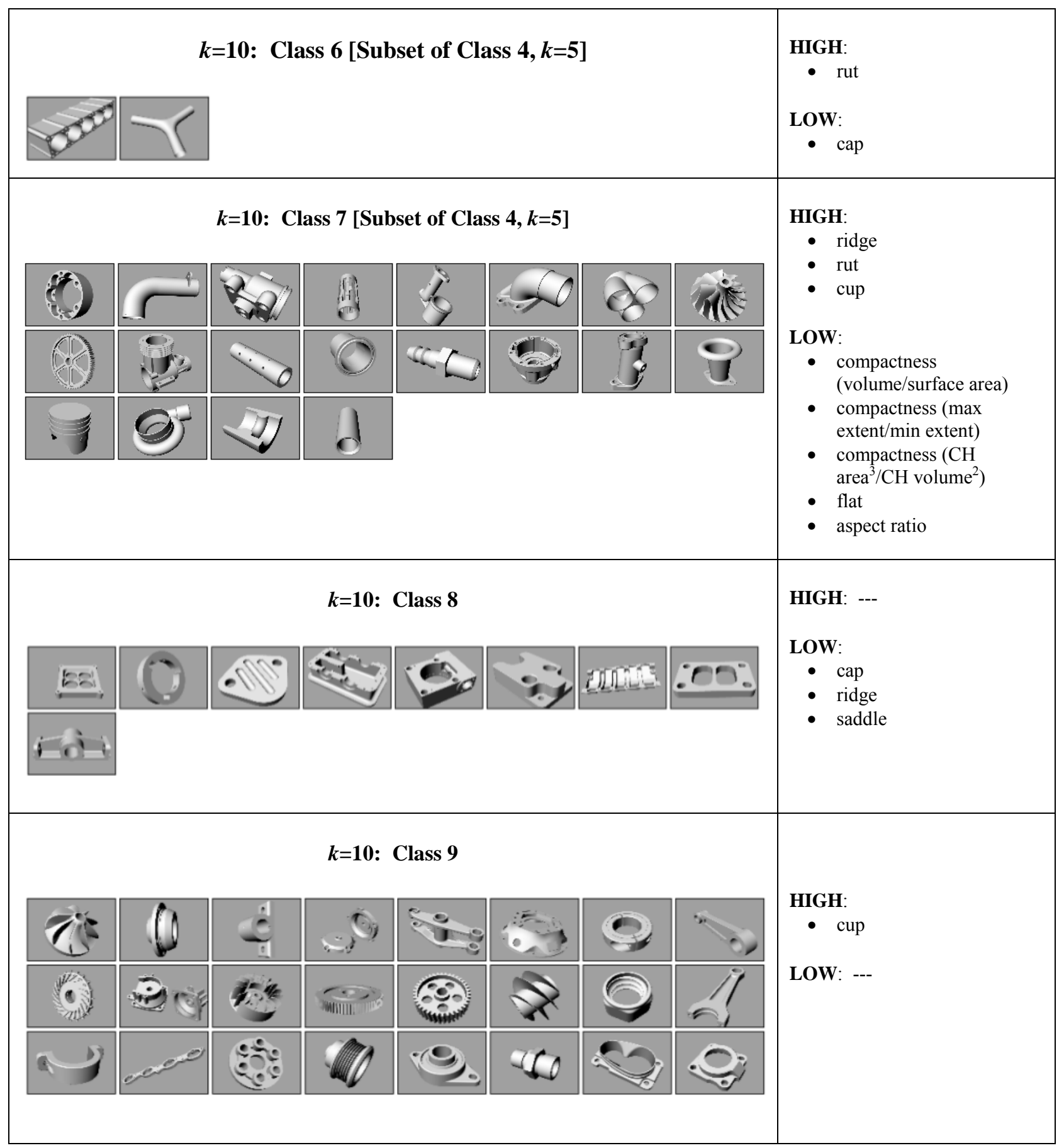




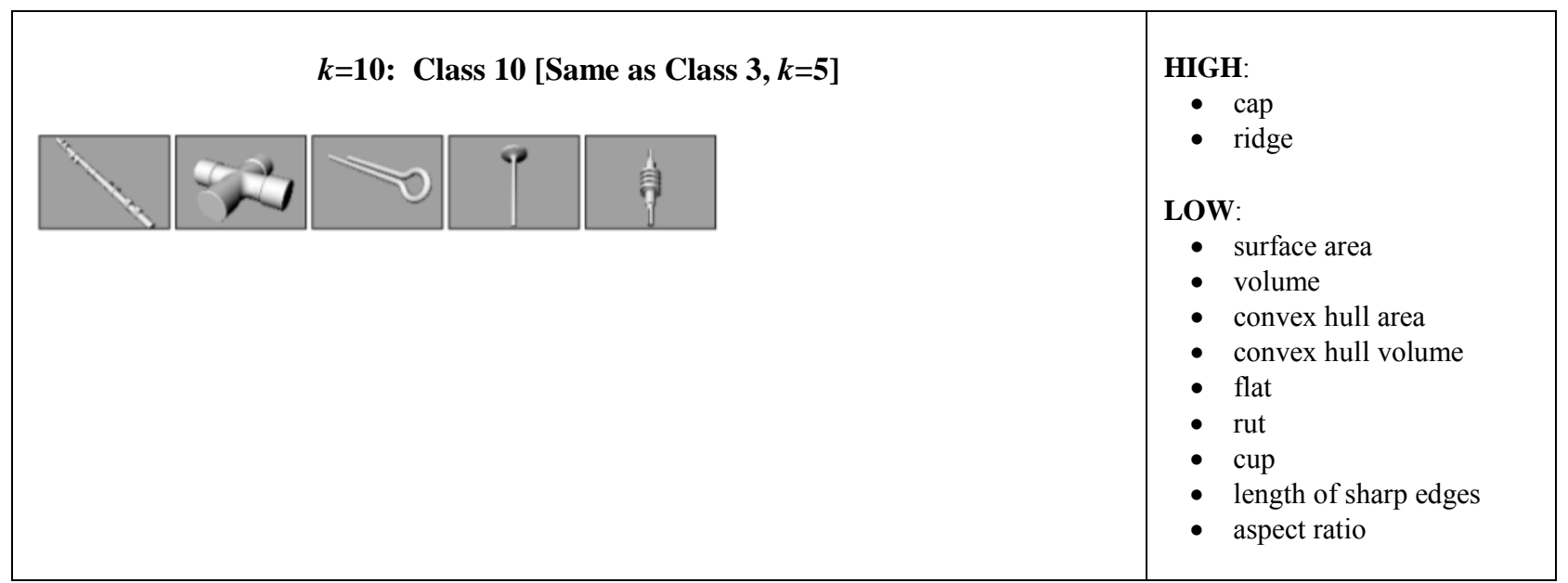




\section{Discussion and Summary}

\subsection{Discussion}

As can be expected, manual classifications will be subjective and will likely lack consistency as evidenced by the classifications in Section 6.1. The subjectivity and lack of consistency may be mitigated by training and developing guidelines/rules for performing the classification.

An objective method to classify objects could be one that is based on metrics characterizing an object. Advantages of such a method are objectivity and ease of implementation. The disadvantages of this method are:

1. Selection and determination of an appropriate set of metrics,

2. Classifications may change if different metrics were used. For example, given an object, the object may be placed in Class 1 using Metrics Set A or it may be placed in Class 2 if Metrics Set B were used,

3. Classifications may change if parts are added to or removed from the population of parts. Clustering methods are based only on the relative similarity of dataset attributes to an aggregate cluster average, and outliers and subsequent changes to the attribute space may cause shifts in cluster centroids,

4. Development of algorithms to quantify the metric. For example, quantifying symmetry is very difficult as discussed in Section 3. Others metrics that would be hard to quantify algorithmically include the number of stable of poses and the number of unique views, and

5. Requirement of a 3D model of the object.

The classifications using cluster analysis (Section 6.2) were performed using only the shape type metrics. For the algorithmic-based approach, the number of classes can be set to any number. To reduce the variables between the algorithmic and the manual approaches, the same number of classes, 5, 6, and 10 used in the manual approaches, were used in the algorithmic-based approach. However, since the criteria for the classifications were different (e.g., geometry based, technology based, shape type) the resulting classifications would not be expected to be similar.

Many methods for clustering are found in various communities. Besides the $k$-means cluster analysis, we also tried a very common approach to clustering called the hierarchical method (Johnson, 1967), also known as Agglomerative clustering. This method is very popular in areas of data mining and bioinformatics. In hierarchical clustering, we do not need to know the number of clusters/groups ahead of time; i.e., the 3D CAD models are not divided into predefined numbers of clusters. We then use a Dendrogram, which is a visual representation of hierarchical data to show the clusters. The Dendrogram tree starts at the root, which is at the top for a vertical tree (the nodes represent clusters). Figure 7.1 shows the Agglomerative clustering based on the shape type metrics only with the number of clusters $k=$ 30 . 


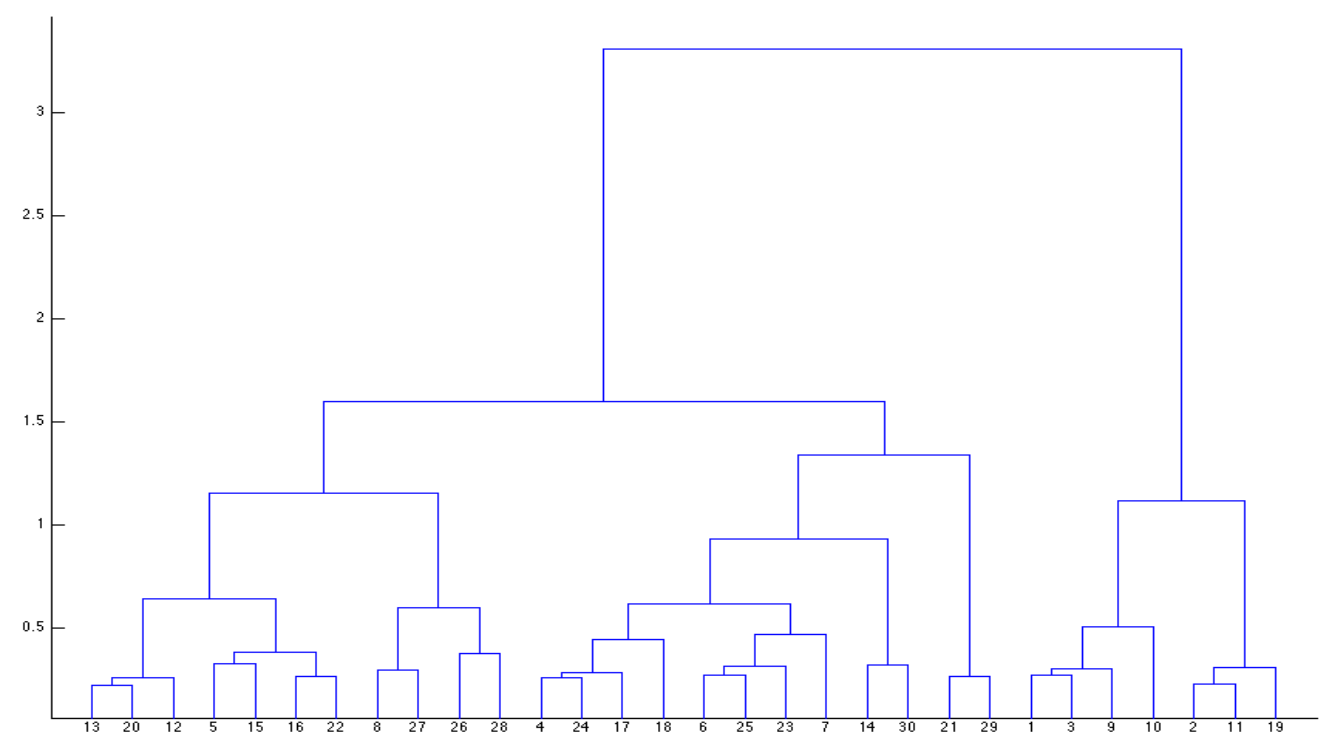

Figure 7.1 Agglomerative clustering based on model shape type metrics (number of clusters $k=30$ ).

In Fig. 7.1, the number of clusters will vary depending on the $y$ value selected. Figure 7.2 shows the clusters for $y \approx 1.1$.

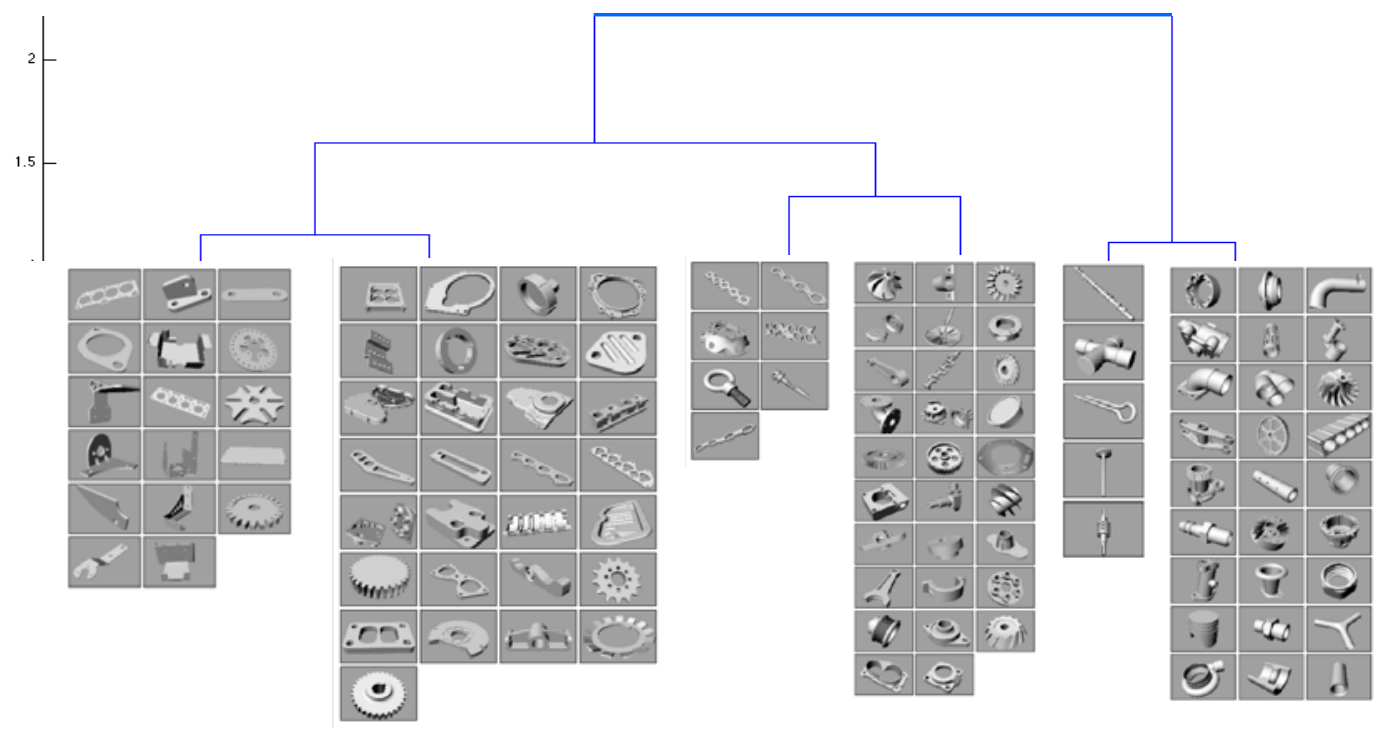

Figure 7.2. Agglomerative clusters for $y \approx 1.1$. 
One other approach for classification is to use a classifier such as a Support Vector Machine (SVM) (Hearst et al., 1998) classifier that is trained by human-generated training examples to classify a test dataset. Other possible classifiers than can be used for classification include neural networks (Funahashi, 1989), naive Bayes classifiers (Kohavi, 1996), and boosted decision stumps (Qu et al., 2002).

The High/Low observations in Section 6 seem to indicate that the density, crumpliness, crinkliness, and length of sharp edges metrics may not be useful as distinguishing/discriminating metrics for the set of objects used in this effort. The area, aspect ratio, cap, compactness, convex hull area, convex hull volume, cup, flat, ridge, and volume metrics may be useful as distinguishing/discriminating metrics.

\subsection{Summary and Future Work}

This study documented the process to develop a classification system for objects. The purpose of the classification system was to support the evaluation of perception systems used for identifying and locating static manufacturing parts. The existence of a robust object classification system may eventually lead to the development of standard reference artifacts of objects in the various classes. A literature review indicated that many of the classification systems developed were for object retrieval and manufacturing systems/process and a classification system such as that envisioned for this study does not exist.

In this study, two approaches, manual and algorithm-based, were used to develop a classification system. Three manual approaches for the classifications were developed where the criterion for one was object geometry and the criteria for the other two were based on the perceived difficulty that a perception system, laser-based and vision-based, would encounter when measuring the pose of the object. The algorithmic approach involved using cluster analysis to classify the objects based on a set of metrics.

A review of metrics used by other researchers was conducted and a set of metrics was selected for use in this study. Commercially or publicly available algorithms were used to calculate the metrics. A database of objects was created where the objects in the database were narrowed down to mainly automotive engine parts and where a 3D CAD model of the object was freely available. In addition to the three manual classifications, $k$-means clustering was used to generate an additional three classifications where $k$ was set equal to 5, 6, and 10 using the calculated metrics for shape type.

Based on this work, developing a classification system such as that proposed in this effort will not be easily accomplished as the problem involves a huge, multidimensional parameter space. However, the experience gained from this work with metrics, algorithms to calculate the metrics, file formats, and object databases will benefit future efforts in dealing with smaller object spaces for more defined applications. Also, the experience will inform other efforts to design artifacts and experiments for object identification and pose estimation.

The next step for this study would be the determination of whether the classification systems in Section 6 are good or bad through experimental testing using various perception systems. A good classification would be one that groups objects into classes for which perception systems would yield similar results for a given class. For example, a classification system could group a set of objects into three classes where the measured positional error for the objects would range from (0 to 10) $\mathrm{mm},(11$ to 20$) \mathrm{mm}$, and (21 to 30) $\mathrm{mm}$ for Class 1, 2, and 3, respectively. An ideal classification system would yield positional errors 
that are within the specified bounds for all objects in that class and for all perception systems. A good classification system would yield positional errors that are within the specified bounds for most of the objects in that class and for most perception systems. A bad classification system would yield positional errors that are outside the specified bounds for the majority of the objects in a given class and for all classes. That is, for more than $50 \%$ of the objects in Class 1 , the errors would be greater than $10 \mathrm{~mm}$ and similarly for objects in Classes 2 and 3.

Evaluation of a classification system requires a set of objects and a perception system. For a given set of objects and a given perception system, the classification system may be deemed good. However, robustness of the classification system is of critical importance - would the classification system fail if other objects were added or if another perception system were used? Also, as stated earlier, metrics for symmetry and the number of significant features were not used in the cluster analysis in this effort. A next step would be to calculate these metrics (and/or other metrics such as number of unique views or color) and determine how the classifications would change and to evaluate the classification system through experimental testing.

If a classification system is deemed bad, the reasons for its failure need to be determined; reasons such as (1) were the metrics not the appropriate ones? and (2) was the classification system only valid for a particular type of perception system technology? 


\section{References}

Alliez, P., Cohen-Steiner, D., Devillers, O., Levy, B., and Desbrun, M., Anisotropic Polygonal Remeshing, Proceedings of ACM SIGGRAPH 2003 papers, July, 2003, pp. 485-493.

Ankerst, M., Kastenmüller, G., Kriegel, H.-P., and Seidl, T., Nearest neighbor classification in 3D protein databases, in Proc. ISMB, 1999.

Bradski, G. and Kaebler, A., "OpenCV”, Newgen Publishing and Data Services, 2008.

Bespalov, D., Ip, C. Y., Regli, W. C., and Shaffer, J., Benchmarking CAD search techniques, SPM '05 Proceedings of the 2005 ACM symposium on Solid and physical modeling, p. 275 - 286.

Bespalov, D., Shokoufandeh, A., Regli, W. C., and Sun, W., Scale-space representation and classification of $3 \mathrm{~d}$ models, JCISE 3 (Dec), 2003, p. 315-324.

Cardone, A., Gupta, S. K., and Karnik, M., A survey of shape similarity assessment algorithms for product design and manufacturing applications, Journal of Computing and Information Science in Engineering, Vol. 3, June, 2003, p. 109-118.

Cicirello, V., and Regli, W. C., An approach to a feature-based comparison of solid models of machined parts, AIEDAM 16, 5 (November), 2002, p.385-399.

Cohen-Steiner, D. and Morvan, J.-M., Restricted Delaunay Triangulations and Normal Cycle, 19th Annual ACM Symposium on Computational Geometry, 2003, pp. 237-246.

Corney J, Rea H, Clark D, Pritchard J, Breaks M, Macleod R (2002), Coarse filters for shape matching, IEEE Comput Graph Appl 22(3):65-74.

Dutagaci, H., Cheung, C. P., Godil, A., Evaluation of 3D interest point detection techniques via human-generated ground truth, The Visual Computer 28, no. 9, 2012, pp. 901-917.

Funahashi, K-I., On the approximate realization of continuous mappings by neural networks, Neural networks 2, no. 3, 1989, pp. 183-192.

Hearst, M. A., Dumais, S. T., , Osman, E., Platt, J., and Scholkopf, B., Support vector machines, Intelligent Systems and their Applications, IEEE 13, no. 4, 1998, pp. 18-28.

Hilaga, M., Shinagawa, Y., Kohmura, T., and Kunii, T. L., Topology matching for fully automatic similarity estimation of 3d shapes, In SIGGRAPH, ACM Press, New York, NY, USA, ACM, 2001, p. 203 - 212.

Horn, B., Extended Gaussian images, Proc. of the IEEE, 72(12):1671-1686, December 1984.

Ip, C. Y., Lapadat, D., Sieger, L., and Regli, W . C., Using shape distributions to compare solid models, in Seventh Symposium on Solid Modeling and Applications, 2002.

Ip, C. Y., Sieger, L., Regli, W. C., and Shokoufandeh, A., Automated learning of model classifications, in Eighth Symposium on Solid Modeling and Applications, 2003, p. 322-27.

Iyer, N., Subramanian, J., Lou, K., Kalyanaraman, Y., and Ramani, K., Three-dimensional shape searching: state-ofthe-art review and future trends, Computer-Aided Design, 37, 2005, p. 509-530.

Jayanti, S., Yagnanarayanan, K., Iyer, N., and Ramani, K., Developing an engineering shape benchmark for CAD models, Computer-Aided Design, 38, 2006, p. 939-953.

Johnson, S. C., Hierarchical clustering schemes, Psychometrika, 32.3, 1967, pp. 241-254.

Kazhdan, M. , Funkhouser, T., and Rusinkiewicz, S., Rotation invariant spherical harmonic representation of 3D shape de-scriptors, in Symposium on Geometry Processing, June 2003.

Kohavi, R., Scaling up the accuracy of naive-Bayes classifiers: A decision-tree hybrid, In $K D D$, August, 1996, pp. 202-207.

McCarthy, I., Manufacturing classification: Lessons from organizational systematics and biological taxonomy, Integrated Manufacturing Systems, Vol. 6, No. 6, 1995, p. 37-48.

Mcwherter, D., Peabody, M., Shokoufandeh, A., and Regli, W., Solid model databases: Techniques and empirical results, JCISE 1, 4 (December), 2001, p. 300-310.

Osada, R., Funkhouser, T., Chazelle, B. and Dobkin, D., Matching 3D models with shape distributions, Shape Modeling International, pages 154-166, May 2001.

Osada, R., Funkhouser, T., Chazelle, B., and Dobkin, D., Shape distributions, ACM Trans. on Graphics 21, 4 (Oct), 2002, p. 807-832.

Peabody, M., Finding Groups of Graphs in Databases, Master's thesis, Drexel University, 2002.

Qu, Y., Adam, B-L., Yasui, Y., Ward, M.. D., Cazares, L. H., Schellhammer, P. F., Feng, Z., Semmes, O. J., and Wright, G. L., Boosted decision tree analysis of surface-enhanced laser desorption/ionization 
mass spectral serum profiles discriminates prostate cancer from noncancer patients, Clinical Chemistry 48, no. 10, 2002, pp. 1835-1843.

Saupe, D. and Vranic, D. V., 3D model retrieval with spherical harmonics and moments, in B. Radig and S. Florczyk, editors, DAGM 2001, pages 392-397, September 2001.

Shilane, P., Min, P., Kazhdan, M., and Funkouser, T., The Princeton shape benchmark, Shape Modeling International, June, 2004.

Rusu, R. and Cousins, S., "3D is here: Point Cloud Library (PCL)", Robotics and Automation (ICRA), 2011 IEEE International Conference on Robotics and Automation, May 9-13, 2011, pp 1-4.

Tangelder, J. W. H. and Veltkamp, R. C. (2008), A survey of content based 3D shape retrieval methods, Multimed Tools App, 39: 441-471.

Teknomo, K., "K-Means Clustering Tutorial." Medicine 100.4, (2006): 3.

Vranic, D. V., An improvement of rotation invariant 3D shape descriptor based on functions on concentric spheres, in IEEE International Conference on Image Processing (ICIP 2003), volume 3, pages 757-760, September 2003.

Zhang, C. and Chen, T., Efficient Feature Extraction for 2D/3D Objects in Mesh Representation, IEEE ICIP, 2001 


\section{Appendix A: Objects Used in Classification Systems}

The objects selected for use in the classification system were downloaded from www.GradCAD.com and images of these objects are given in the table below. The object file names are given below each image, and they indicate the format of each file: SLDPRT = SolidWorks; STP/STEP = Step; IGS/IGES = IGES; $3 \mathrm{DM}=$ Rhino; $\mathrm{DWG}=$ AutoCAD.

The objects in red and blue shaded cells were not included in the classification. The cells shaded in red could not be meshed. The curvature metrics for objects in cells shaded in blue could not be calculated. There are some known bugs in the curvature algorithm and further debugging of the algorithm was not pursued as this was beyond the scope of the task. The orange shaded cells were objects which had at least one dimension greater than $50 \mathrm{~cm}$.

Table A.1. Objects used in the classification systems.

\begin{tabular}{|c|c|c|c|}
\hline 1inchCarbSpacer.SLDPRT & $\begin{array}{l}4 \text { cylinder engine head } \\
\text { gasket.SLDPRT }\end{array}$ & $\begin{array}{l}944 \text { Bellhousing } \\
\text { Base.SLDPRT }\end{array}$ & $\begin{array}{l}\text { 944to911OuterCVAdapter.S } \\
\text { LDPRT } \\
\end{array}$ \\
\hline 9 Blade Impeller.SLDPRT & 9 Inch Pin Support.SLDPRT & AlumPIPE.SLDPRT & $\begin{array}{l}\text { Audi20VExhaustFlange.SL } \\
\text { DPRT }\end{array}$ \\
\hline $\begin{array}{l}\text { Audi bearing retainer } \\
\text { tool.SLDPRT }\end{array}$ & $\begin{array}{c}\text { Audi-VW Rear crank } \\
\text { plate.IGS }\end{array}$ & Base Bracket.SLDPRT & bearing body.SLDPRT \\
\hline Bevel Gear.SLDPRT & BigBlockChevyFlange.igs & Block.dwg & boltnew.SLDPRT \\
\hline
\end{tabular}




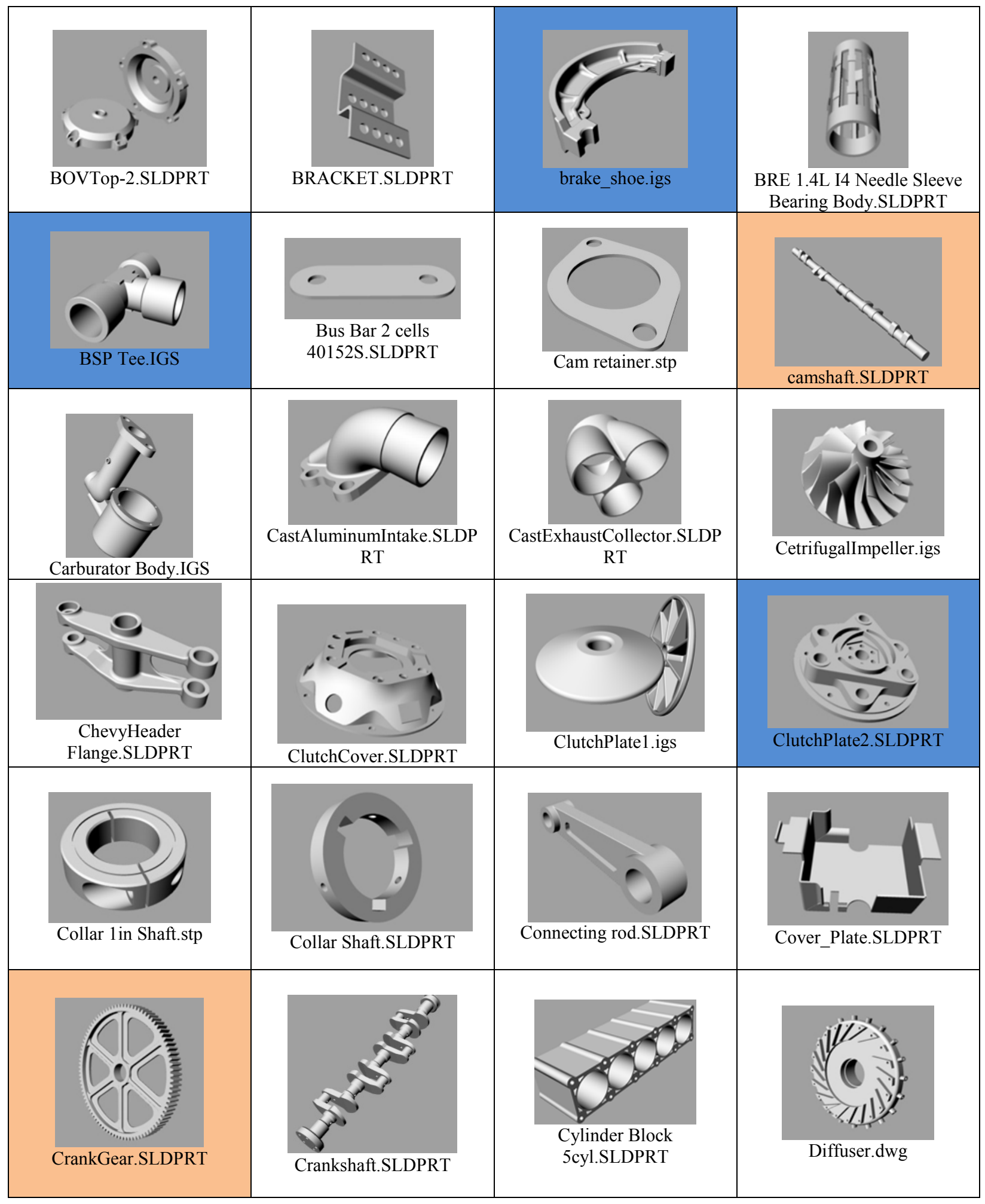




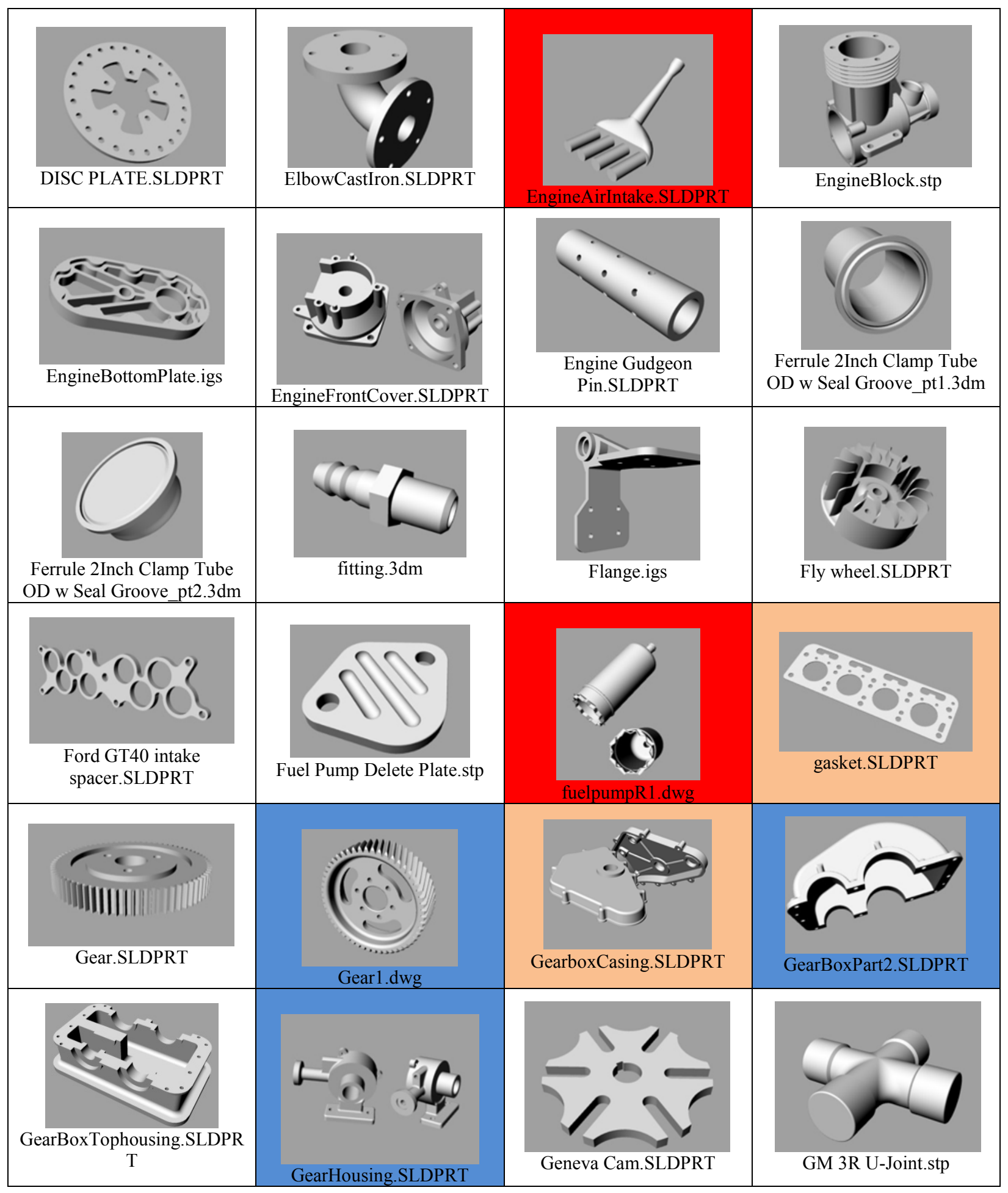




\begin{tabular}{|c|c|c|c|}
\hline GMLS6FrtCover.SLDPRT & Helical gear wheel.STEP & $\begin{array}{c}\text { HondaNSR125CylinderHead } \\
\text { Gasket.IGS }\end{array}$ & Housing.SLDPRT \\
\hline HousingSquare.SLDPRT & $\begin{array}{l}\text { IgnitionCoilMazda13B.SLD } \\
\text { PRT }\end{array}$ & Impeller.SLDPRT & IntakeManifold. $3 \mathrm{dm}$ \\
\hline $\begin{array}{l}\text { IntakeMobil.IGS } \\
\text { (IntakeMobilPart1.stl) }\end{array}$ & $\begin{array}{c}\text { IntakeMobil.IGS } \\
\text { (IntakeMobilPart2.stl) }\end{array}$ & IntakePipeComponent.igs & IntakeTrumpet.igs \\
\hline Bracket.SLDPRT & link.IGS & LockNuts.SLDPRT & LowerControlArm.stp \\
\hline $\begin{array}{l}\text { LowerThermostatHousing } \\
\text { spacer GM.SLDPRT }\end{array}$ & $\begin{array}{l}\text { M20LiftingEyeBolt.SLDPR } \\
\mathrm{T}\end{array}$ & ManifoldPlate.stp & MasterCylinder Bracket.3dm \\
\hline $\begin{array}{c}\text { Miata16IntakeFlange.SLDP } \\
\text { RT }\end{array}$ & $\begin{array}{l}\text { MitsubishiEVOMAPadapter. } \\
\text { SLDPRT }\end{array}$ & $\begin{array}{l}\text { MitsubishiGrandisAirIntake. } \\
\text { stp }\end{array}$ & $\begin{array}{c}\text { Needle valve } \\
\text { screw.SLDPRT }\end{array}$ \\
\hline
\end{tabular}




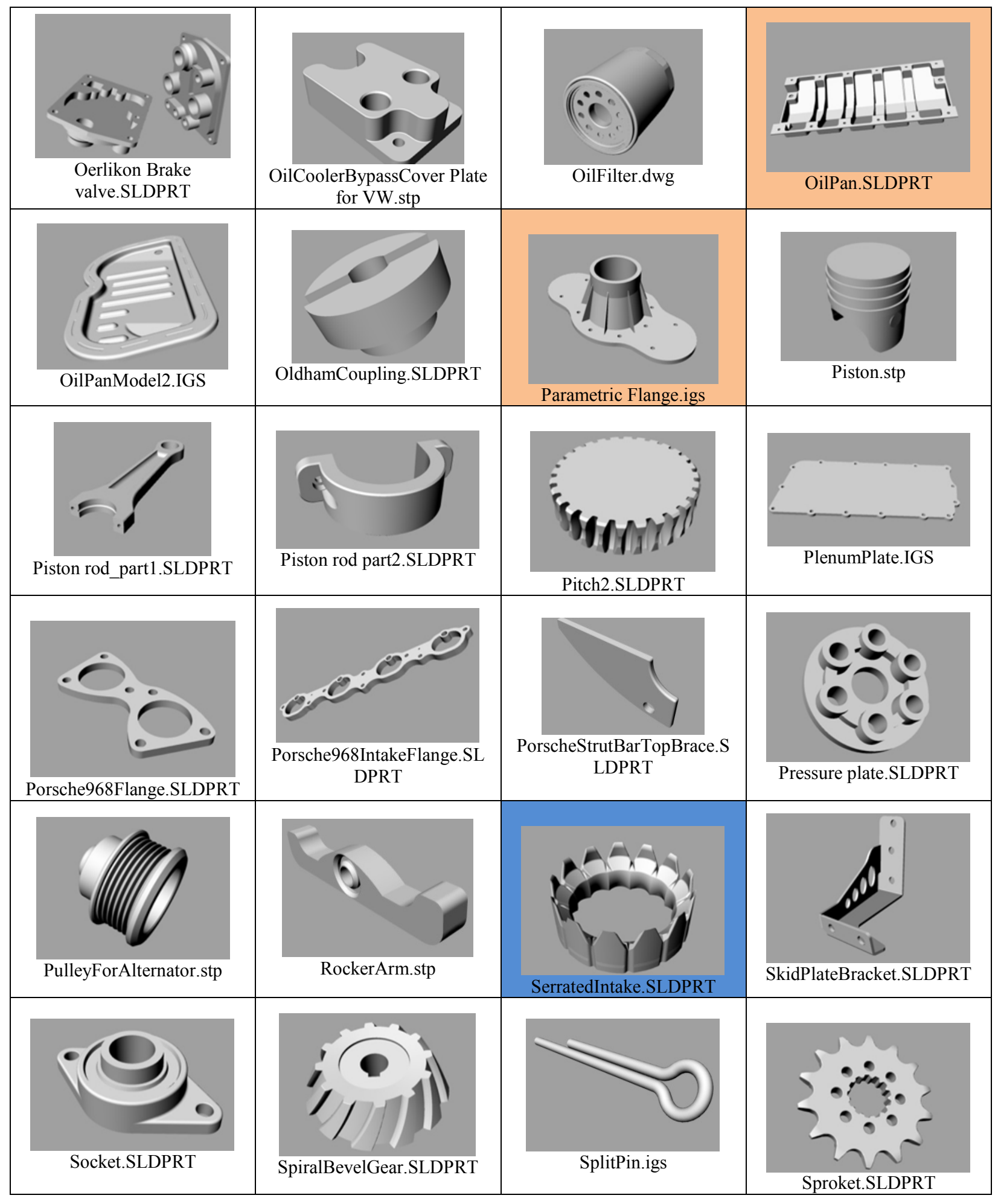




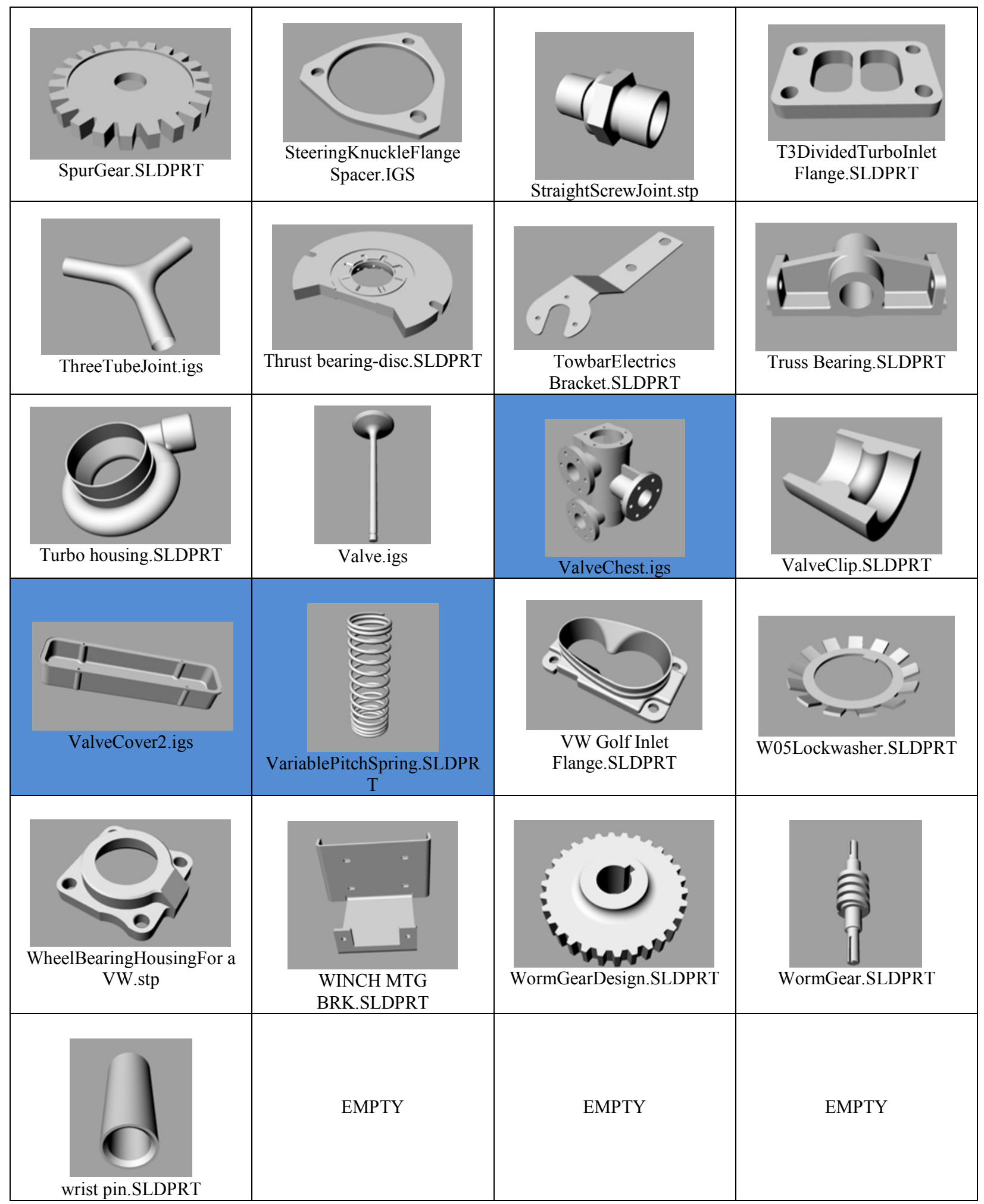




\section{Appendix B: Calculated Metrics for Objects in Appendix A.}

Table B1. Calculated Metrics

\begin{tabular}{|c|c|c|c|c|c|c|c|c|c|c|c|c|}
\hline \multirow{2}{*}{ FileName } & \multirow{2}{*}{$\begin{array}{l}\text { Object } \\
\text { ID }\end{array}$} & \multirow{2}{*}{$\begin{array}{l}\text { Surface } \\
\text { Area } \\
\text { [cm2] }\end{array}$} & \multirow{2}{*}{$\begin{array}{l}\text { Vol } \\
{[\mathrm{cm} 3]}\end{array}$} & \multicolumn{3}{|c|}{ Extent } & \multirow{2}{*}{$\begin{array}{c}\text { Convex } \\
\text { Hull } \\
\text { Area } \\
{\left[\mathrm{cm}^{2}\right]}\end{array}$} & \multirow{2}{*}{$\begin{array}{c}\text { Convex } \\
\text { Hull } \\
\text { Vol } \\
{\left[\mathbf{c m}^{3}\right]}\end{array}$} & \multirow{2}{*}{$\begin{array}{c}\text { Compactness } \\
\text { Vol/Surface } \\
\text { Area } \\
\text { [cm] }\end{array}$} & \multirow{2}{*}{$\begin{array}{c}\text { Compactness } \\
\text { Max/Min } \\
\text { Extent }\end{array}$} & \multirow{2}{*}{$\begin{array}{c}\text { Compactness } \\
\text { CHarea }^{3} / \text { CHvol }^{2}\end{array}$} & \multirow{2}{*}{$\begin{array}{c}\text { Density } \\
1-\text { Vol/ChVol }\end{array}$} \\
\hline & & & & $\begin{array}{c}\mathrm{DX} \\
{[\mathrm{cm}]}\end{array}$ & $\begin{array}{c}\text { DY } \\
{[\mathrm{cm}]}\end{array}$ & $\begin{array}{c}\mathrm{DZ} \\
{[\mathrm{cm}]}\end{array}$ & & & & & & \\
\hline 1inchCarbSpacer & 1 & 603 & 331 & 15 & 3 & 16 & 639 & 615 & 0.549 & 6.38 & 688.44 & 0.46 \\
\hline 4cylinderEngineHeadGasket & 2 & 641 & 44 & 40 & 0 & 17 & 1308 & 97 & 0.069 & 269.86 & 238461.05 & 0.54 \\
\hline 944BellhousingBase & 3 & 1307 & 830 & 37 & 2 & 34 & 2065 & 1773 & 0.635 & 19.25 & 2800.11 & 0.53 \\
\hline 944to911OuterCVAdapter & 4 & 373 & 121 & 4 & 11 & 11 & 300 & 338 & 0.324 & 2.87 & 235.50 & 0.64 \\
\hline 9BladeImpeller & 5 & 1 & 0 & 1 & 0 & 1 & 1 & 0 & 0.024 & 1.87 & 178.78 & 0.45 \\
\hline 9InchPinSupport & 6 & 779 & 549 & 17 & 7 & 17 & 588 & 1092 & 0.705 & 2.27 & 170.33 & 0.50 \\
\hline AlumPIPE & 7 & 1517 & 301 & 27 & 11 & 17 & 1080 & 2470 & 0.198 & 2.51 & 206.58 & 0.88 \\
\hline Audi20VExhaustFlange & 8 & 558 & 143 & 43 & 9 & 1 & 796 & 287 & 0.256 & 53.75 & 6108.84 & 0.50 \\
\hline AudiBearingRetainerTool & 9 & 211 & 94 & 8 & 6 & 8 & 183 & 194 & 0.447 & 1.38 & 163.36 & 0.51 \\
\hline AudiVWRearCrankPlate & 10 & 291 & 62 & 15 & 2 & 13 & 379 & 240 & 0.214 & 9.56 & 938.77 & 0.74 \\
\hline BaseBracket & 11 & 405 & 269 & 14 & 9 & 7 & 387 & 467 & 0.664 & 2.13 & 265.91 & 0.42 \\
\hline BearingBody & 12 & 968 & 908 & 10 & 11 & 23 & 833 & 1611 & 0.938 & 2.30 & 222.29 & 0.44 \\
\hline BevelGear & 13 & 250 & 116 & 3 & 11 & 11 & 211 & 185 & 0.462 & 3.94 & 276.91 & 0.37 \\
\hline BigBlockChevyFlange & 14 & 488 & 138 & 48 & 8 & 1 & 779 & 324 & 0.283 & 50.69 & 4493.55 & 0.57 \\
\hline Block & 15 & 271 & 80 & 9 & 6 & 6 & 177 & 187 & 0.296 & 1.50 & 158.12 & 0.57 \\
\hline BOVTop2 & 16 & 153 & 38 & 8 & 2 & 8 & 128 & 73 & 0.251 & 4.75 & 392.98 & 0.48 \\
\hline BRACKET & 17 & 398 & 75 & 10 & 3 & 15 & 394 & 353 & 0.188 & 4.69 & 491.85 & 0.79 \\
\hline $\begin{array}{c}\text { BRE14LI4NeedleSleeve } \\
\text { BearingBody }\end{array}$ & 18 & 43 & 2 & 2 & 2 & 5 & 35 & 14 & 0.045 & 2.66 & 217.75 & 0.86 \\
\hline BusBar2Cells40152S & 19 & 25 & 2 & 6 & 0 & 2 & 25 & 2 & 0.067 & 40.73 & 5254.05 & 0.06 \\
\hline CamRetainer & 20 & 32 & 2 & 7 & 5 & 0 & 51 & 4 & 0.066 & 44.69 & 8937.25 & 0.46 \\
\hline camshaft & 21 & 404 & 193 & 4 & 51 & 4 & 516 & 385 & 0.477 & 12.50 & 925.38 & 0.50 \\
\hline
\end{tabular}




\begin{tabular}{|c|c|c|c|c|c|c|c|c|c|c|c|c|}
\hline CarburatorBody & 22 & 974 & 282 & 9 & 18 & 18 & 725 & 1378 & 0.290 & 2.07 & 200.83 & 0.80 \\
\hline CastAluminumIntake & 23 & 525 & 99 & 15 & 10 & 10 & 389 & 499 & 0.189 & 1.52 & 236.53 & 0.80 \\
\hline CastExhaustCollector & 24 & 361 & 34 & 9 & 6 & 8 & 245 & 291 & 0.093 & 1.41 & 174.61 & 0.88 \\
\hline CetrifugalImpeller & 25 & 185 & 21 & 7 & 3 & 7 & 97 & 66 & 0.114 & 2.10 & 207.82 & 0.68 \\
\hline ChevyHeaderFlange & 26 & 554 & 142 & 21 & 5 & 10 & 512 & 646 & 0.256 & 4.67 & 321.56 & 0.78 \\
\hline ClutchCover & 27 & 1340 & 248 & 24 & 6 & 24 & 1130 & 2028 & 0.185 & 4.00 & 351.20 & 0.88 \\
\hline ClutchPlate1 & 28 & 562 & 132 & 15 & 15 & 3 & 390 & 281 & 0.235 & 6.00 & 754.82 & 0.53 \\
\hline Collar1 inShaft & 29 & 55 & 12 & 4 & 4 & 1 & 48 & 20 & 0.212 & 3.50 & 281.39 & 0.41 \\
\hline CollarShaft & 30 & 67 & 14 & 6 & 1 & 6 & 75 & 28 & 0.211 & 6.00 & 536.16 & 0.50 \\
\hline ConnectingRod & 31 & 284 & 101 & 18 & 3 & 5 & 264 & 211 & 0.355 & 6.07 & 412.31 & 0.52 \\
\hline CoverPlate & 32 & 1454 & 210 & 25 & 11 & 19 & 1305 & 3306 & 0.144 & 2.36 & 203.14 & 0.94 \\
\hline CrankGear & 33 & 11864 & 9378 & 78 & 7 & 78 & 11315 & 34789 & 0.790 & 10.67 & 1196.91 & 0.73 \\
\hline Crankshaft & 34 & 759 & 305 & 5 & 9 & 40 & 840 & 1102 & 0.402 & 7.99 & 488.22 & 0.72 \\
\hline CylinderBlock5cyl & 35 & 7361 & 3062 & 49 & 16 & 12 & 3115 & 9465 & 0.416 & 4.12 & 337.37 & 0.68 \\
\hline Diffuser & 36 & 1197 & 511 & 6 & 21 & 21 & 880 & 1570 & 0.427 & 3.38 & 276.40 & 0.67 \\
\hline DISCPLATE & 37 & 692 & 202 & 22 & 1 & 22 & 784 & 279 & 0.292 & 28.33 & 6189.29 & 0.28 \\
\hline ElbowCastIron & 38 & 2978 & 2488 & 23 & 26 & 26 & 2214 & 7295 & 0.836 & 1.17 & 204.07 & 0.66 \\
\hline EngineBlock & 39 & 645 & 132 & 8 & 11 & 11 & 362 & 538 & 0.205 & 1.46 & 164.36 & 0.75 \\
\hline EngineBottomPlate & 40 & 524 & 162 & 2 & 20 & 10 & 460 & 357 & 0.309 & 10.00 & 762.94 & 0.55 \\
\hline EngineFrontCover & 41 & 264 & 77 & 9 & 9 & 5 & 207 & 225 & 0.293 & 1.73 & 176.55 & 0.66 \\
\hline EngineGudgeonPin & 42 & 40 & 4 & 5 & 1 & 1 & 25 & 8 & 0.091 & 3.57 & 265.98 & 0.52 \\
\hline $\begin{array}{c}\text { Ferrule2 InchClampTubeO } \\
\text { DwSealGroovePt1 }\end{array}$ & 43 & 208 & 19 & 6 & 6 & 6 & 158 & 152 & 0.089 & 1.12 & 170.49 & 0.88 \\
\hline $\begin{array}{c}\text { Ferrule2InchClampTubeO } \\
\text { DwSealGroovePt2 } \\
\end{array}$ & 44 & 101 & 45 & 2 & 6 & 6 & 94 & 57 & 0.451 & 3.01 & 250.53 & 0.21 \\
\hline fitting & 45 & 33 & 4 & 5 & 2 & 2 & 24 & 8 & 0.108 & 2.71 & 203.28 & 0.56 \\
\hline Flange & 46 & 322 & 73 & 8 & 13 & 12 & 378 & 510 & 0.227 & 1.68 & 207.52 & 0.86 \\
\hline FlyWheel & 47 & 348 & 92 & 9 & 4 & 9 & 218 & 228 & 0.265 & 2.34 & 200.38 & 0.60 \\
\hline FordGT40IntakeSpacer & 48 & 410 & 95 & 34 & 1 & 9 & 638 & 267 & 0.232 & 35.31 & 3636.82 & 0.64 \\
\hline FuelPumpDeletePlate & 49 & 61 & 12 & 6 & 5 & 1 & 56 & 14 & 0.201 & 10.00 & 853.14 & 0.15 \\
\hline gasket & 50 & 2795 & 390 & 80 & 25 & 0 & 4036 & 596 & 0.140 & 266.67 & 184956.67 & 0.35 \\
\hline
\end{tabular}




\begin{tabular}{|c|c|c|c|c|c|c|c|c|c|c|c|c|}
\hline Gear & 51 & 563 & 244 & 14 & 14 & 2 & 416 & 325 & 0.433 & 7.20 & 679.26 & 0.25 \\
\hline GearboxCasing & 52 & 6386 & 3046 & 66 & 45 & 8 & 5254 & 14064 & 0.477 & 8.72 & 733.23 & 0.78 \\
\hline GearBoxTophousing & 53 & 5946 & 6377 & 48 & 28 & 12 & 4105 & 14760 & 1.072 & 4.00 & 317.42 & 0.57 \\
\hline GenevaCam & 54 & 317 & 107 & 14 & 15 & 1 & 367 & 161 & 0.338 & 14.54 & 1911.01 & 0.33 \\
\hline GM3RUJoint & 55 & 154 & 94 & 9 & 9 & 3 & 187 & 170 & 0.611 & 3.22 & 227.41 & 0.45 \\
\hline GMLS6FrtCover & 56 & 1708 & 401 & 32 & 27 & 5 & 1527 & 2184 & 0.235 & 6.18 & 745.99 & 0.82 \\
\hline HelicalGearWheel & 57 & 779 & 225 & 14 & 14 & 4 & 483 & 615 & 0.289 & 3.50 & 298.25 & 0.63 \\
\hline $\begin{array}{c}\text { HondaNSR125Cylinder } \\
\text { HeadGasket }\end{array}$ & 58 & 93 & 1 & 10 & 10 & 0 & 153 & 2 & 0.012 & 328.54 & 659909.64 & 0.53 \\
\hline Housing & 59 & 644 & 209 & 11 & 11 & 8 & 390 & 601 & 0.325 & 1.34 & 164.57 & 0.65 \\
\hline HousingSquare & 60 & 971 & 575 & 15 & 14 & 6 & 702 & 1144 & 0.592 & 2.47 & 264.25 & 0.50 \\
\hline IgnitionCoilMazda13B & 61 & 194 & 73 & 4 & 9 & 9 & 164 & 142 & 0.375 & 2.60 & 221.13 & 0.49 \\
\hline Impeller & 62 & 288 & 63 & 8 & 6 & 8 & 175 & 208 & 0.218 & 1.33 & 123.36 & 0.70 \\
\hline IntakeMobilPart1 & 63 & 1819 & 1014 & 33 & 9 & 8 & 1230 & 2270 & 0.557 & 4.41 & 361.16 & 0.55 \\
\hline IntakePipeComponent-1 & 64 & 176 & 32 & 6 & 5 & 8 & 142 & 122 & 0.183 & 1.59 & 194.76 & 0.74 \\
\hline IntakeTrumpet & 65 & 82 & 3 & 4 & 4 & 4 & 64 & 42 & 0.032 & 1.06 & 153.80 & 0.94 \\
\hline JettaActuatorBracket & 66 & 106 & 15 & 8 & 6 & 5 & 122 & 78 & 0.137 & 1.52 & 297.09 & 0.81 \\
\hline link & 67 & 727 & 402 & 40 & 13 & 2 & 835 & 670 & 0.553 & 20.21 & 1298.01 & 0.40 \\
\hline LockNuts & 68 & 12 & 1 & 2 & 2 & 1 & 9 & 2 & 0.063 & 1.75 & 168.47 & 0.64 \\
\hline $\begin{array}{c}\text { LowerThermostatHousing } \\
\text { SpacerGM }\end{array}$ & 69 & 103 & 24 & 12 & 3 & 1 & 110 & 39 & 0.233 & 12.83 & 876.92 & 0.38 \\
\hline M20LiftingEyeBolt & 70 & 167 & 58 & 7 & 12 & 3 & 157 & 117 & 0.349 & 4.03 & 282.70 & 0.50 \\
\hline ManifoldPlate & 71 & 430 & 149 & 33 & 7 & 1 & 507 & 250 & 0.347 & 27.54 & 2081.82 & 0.40 \\
\hline MasterCylinderBracket & 72 & 559 & 88 & 13 & 9 & 13 & 627 & 1037 & 0.157 & 1.48 & 229.36 & 0.92 \\
\hline Miata16IntakeFlange & 73 & 483 & 137 & 34 & 9 & 1 & 629 & 264 & 0.284 & 36.22 & 3586.81 & 0.48 \\
\hline MitsubishiEVOMAPadapter & 74 & 58 & 13 & 6 & 2 & 3 & 47 & 24 & 0.233 & 2.52 & 191.90 & 0.43 \\
\hline NeedleValveScrew & 75 & 5 & 0 & 1 & 3 & 1 & 5 & 1 & 0.064 & 4.70 & 309.13 & 0.47 \\
\hline OerlikonBrakeValve & 76 & 1606 & 812 & 18 & 6 & 24 & 1126 & 2008 & 0.505 & 4.29 & 354.10 & 0.60 \\
\hline $\begin{array}{c}\text { OilCoolerBypasCover } \\
\text { PlateForVW } \\
\end{array}$ & 77 & 217 & 111 & 9 & 6 & 3 & 184 & 144 & 0.512 & 3.50 & 300.79 & 0.23 \\
\hline OilFilter & 78 & 1533 & 2768 & 12 & 15 & 12 & 710 & 1535 & 1.806 & 1.26 & 152.17 & $-0.80^{1}$ \\
\hline OilPan & 79 & 4452 & 1217 & 51 & 8 & 24 & 3097 & 6854 & 0.273 & 6.43 & 632.10 & 0.82 \\
\hline
\end{tabular}




\begin{tabular}{|c|c|c|c|c|c|c|c|c|c|c|c|c|}
\hline OilPanModel2 & 80 & 1546 & 123 & 19 & 3 & 34 & 1259 & 1043 & 0.080 & 13.45 & 1835.42 & 0.88 \\
\hline OldhamCoupling & 81 & 775 & 850 & 15 & 8 & 15 & 638 & 1221 & 1.097 & 1.96 & 174.25 & 0.30 \\
\hline ParametricFlange & 82 & 23526 & 42614 & 135 & 68 & 43 & 19839 & 167630 & 1.811 & 3.18 & 277.87 & 0.75 \\
\hline piston & 83 & 655 & 420 & 11 & 11 & 11 & 480 & 850 & 0.642 & 1.05 & 153.62 & 0.51 \\
\hline PistonRodPart1 & 84 & 196 & 57 & 6 & 17 & 2 & 221 & 121 & 0.294 & 11.50 & 741.03 & 0.52 \\
\hline PistonRodPart2 & 85 & 145 & 47 & 11 & 6 & 3 & 165 & 130 & 0.322 & 3.67 & 264.93 & 0.64 \\
\hline pitch2 & 86 & 108 & 40 & 6 & 6 & 2 & 86 & 46 & 0.371 & 3.82 & 304.58 & 0.13 \\
\hline PlenumPlate & 87 & 1409 & 418 & 40 & 1 & 18 & 1466 & 444 & 0.297 & 63.19 & 16026.04 & 0.06 \\
\hline Porsche968Flange & 88 & 202 & 52 & 17 & 8 & 1 & 291 & 118 & 0.257 & 18.35 & 1769.83 & 0.56 \\
\hline Porsche968IntakeFlange & 89 & 573 & 186 & 46 & 2 & 7 & 715 & 447 & 0.325 & 28.94 & 1832.44 & 0.58 \\
\hline PorscheStrutBarTopBrace & 90 & 87 & 18 & 11 & 1 & 5 & 89 & 19 & 0.207 & 21.79 & 1966.39 & 0.05 \\
\hline PressurePlate & 91 & 176 & 41 & 8 & 2 & 8 & 126 & 80 & 0.236 & 4.00 & 310.38 & 0.48 \\
\hline PulleyForAlternator & 92 & 286 & 63 & 6 & 7 & 7 & 161 & 174 & 0.221 & 1.17 & 137.78 & 0.64 \\
\hline RockerArm & 93 & 758 & 695 & 4 & 36 & 7 & 801 & 951 & 0.916 & 9.00 & 568.10 & 0.27 \\
\hline SkidPlateBracket & 94 & 495 & 71 & 16 & 19 & 12 & 649 & 918 & 0.144 & 1.52 & 324.72 & 0.92 \\
\hline socket & 95 & 280 & 153 & 14 & 8 & 4 & 232 & 234 & 0.549 & 3.38 & 226.56 & 0.35 \\
\hline SpiralBevelGear & 96 & 269 & 175 & 11 & 3 & 11 & 245 & 217 & 0.652 & 3.67 & 313.46 & 0.19 \\
\hline SplitPin & 97 & 2 & 0 & 0 & 3 & 1 & 3 & 0 & 0.031 & 25.08 & 1225.96 & 0.49 \\
\hline sproket & 98 & 0 & 0 & 0 & 0 & 0 & 0 & 0 & 0.008 & 8.54 & 862.56 & 0.42 \\
\hline SpurGear & 99 & 449 & 154 & 15 & 15 & 2 & 411 & 256 & 0.343 & 9.82 & 1058.35 & 0.40 \\
\hline SteeringKnuckleFlangeSpacer & 100 & 155 & 98 & 14 & 14 & 1 & 294 & 85 & 0.631 & 22.07 & 3541.99 & $-0.15^{1}$ \\
\hline StraightScrewJoint & 101 & 28 & 4 & 3 & 2 & 2 & 21 & 8 & 0.154 & 1.58 & 158.40 & 0.42 \\
\hline T3DividedTurboInletFlange & 102 & 181 & 56 & 11 & 1 & 7 & 185 & 90 & 0.311 & 8.50 & 771.25 & 0.38 \\
\hline ThreeTubeJoint & 103 & 137 & 51 & 14 & 12 & 2 & 258 & 203 & 0.375 & 5.65 & 418.39 & 0.75 \\
\hline ThrustBearingDisc & 104 & 71 & 11 & 6 & 5 & 1 & 69 & 16 & 0.160 & 11.82 & 1290.09 & 0.28 \\
\hline TowbarElectricsBracket & 105 & 311 & 29 & 8 & 30 & 4 & 440 & 282 & 0.094 & 8.27 & 1070.98 & 0.90 \\
\hline TrussBearing & 106 & 817 & 495 & 21 & 7 & 9 & 634 & 1025 & 0.606 & 3.05 & 242.18 & 0.52 \\
\hline TurboHousing & 107 & 4828 & 1970 & 37 & 16 & 34 & 2806 & 10953 & 0.408 & 2.28 & 184.07 & 0.82 \\
\hline valve & 108 & 35 & 7 & 4 & 4 & 10 & 78 & 41 & 0.191 & 2.91 & 281.38 & 0.84 \\
\hline ValveClip & 109 & 2 & 0 & 1 & 1 & 0 & 2 & 0 & 0.044 & 2.00 & 238.07 & 0.47 \\
\hline
\end{tabular}




\begin{tabular}{|c|c|c|c|c|c|c|c|c|c|c|c|c|}
\hline VWGolfInletFlange & 110 & 207 & 40 & 11 & 7 & 3 & 202 & 158 & 0.192 & 4.52 & 331.82 & 0.75 \\
\hline W05Lockwasher & 111 & 17 & 1 & 4 & 4 & 0 & 29 & 4 & 0.040 & 13.40 & 1809.52 & 0.82 \\
\hline WheelBearingHousingForAVW & 112 & 237 & 61 & 9 & 9 & 3 & 208 & 175 & 0.259 & 3.41 & 292.83 & 0.65 \\
\hline WINCHMTGBRK & 113 & 1644 & 484 & 25 & 15 & 21 & 1875 & 5308 & 0.294 & 1.63 & 234.10 & 0.91 \\
\hline WormGear & 114 & 437 & 271 & 6 & 6 & 28 & 405 & 484 & 0.620 & 4.67 & 284.50 & 0.44 \\
\hline WormGearDesign & 115 & 2075 & 3160 & 28 & 28 & 10 & 1718 & 4758 & 1.523 & 2.80 & 224.13 & 0.34 \\
\hline WristPin & 116 & 84 & 13 & 2 & 2 & 6 & 56 & 28 & 0.151 & 2.70 & 225.81 & 0.54 \\
\hline
\end{tabular}

Table B1. Calculated Metrics (continued)

\begin{tabular}{|c|c|c|c|c|c|c|c|c|c|c|c|c|}
\hline FileName & $\begin{array}{c}\text { Smoothness: } \\
\text { Hull } \\
\text { Crumpliness } \\
\text { Surface } \\
\text { Area/ } \\
\text { CHarea }\end{array}$ & $\begin{array}{c}\text { Smoothness: } \\
\text { Crinkliness } \\
\text { Ratio of surface area } \\
\text { to surface area of } \\
\text { sphere with same } \\
\text { volume as object }\end{array}$ & Cap & Ridge & Flat & Saddle & Rut & Cup & $\begin{array}{l}\text { Normalized } \\
\text { Length of } \\
\text { Sharp Edges }\end{array}$ & DX/DY & DX/DZ & DY/DZ \\
\hline 1inchCarbSpacer & 0.94 & 2.60 & 0.012 & 0.125 & 0.598 & 0.059 & 0.199 & 0.007 & 13.02 & 5.91 & 0.93 & 0.16 \\
\hline 4cylinderEngineHeadGasket & 0.49 & 10.58 & 0.019 & 0.174 & 0.697 & 0.110 & 0.000 & 0.000 & 8.58 & 269.86 & 2.37 & 0.01 \\
\hline 944BellhousingBase & 0.63 & 3.06 & 0.008 & 0.172 & 0.624 & 0.056 & 0.139 & 0.000 & 5.99 & 19.25 & 1.07 & 0.06 \\
\hline 944to911OuterCVAdapter & 1.24 & 3.16 & 0.017 & 0.367 & 0.129 & 0.062 & 0.407 & 0.018 & 13.40 & 0.35 & 0.35 & 1.00 \\
\hline 9BladeImpeller & 1.49 & 2.60 & 0.022 & 0.272 & 0.370 & 0.050 & 0.284 & 0.001 & 11.89 & 1.87 & 1.00 & 0.54 \\
\hline 9InchPinSupport & 1.32 & 2.40 & 0.027 & 0.367 & 0.275 & 0.080 & 0.233 & 0.016 & 16.51 & 2.27 & 1.00 & 0.44 \\
\hline AlumPIPE & 1.40 & 6.98 & 0.111 & 0.358 & 0.016 & 0.103 & 0.331 & 0.082 & 3.76 & 2.51 & 1.54 & 0.61 \\
\hline Audi20VExhaustFlange & 0.70 & 4.23 & 0.104 & 0.180 & 0.351 & 0.322 & 0.040 & 0.004 & 9.57 & 4.89 & 53.75 & 11.00 \\
\hline AudiBearingRetainerTool & 1.15 & 2.11 & 0.018 & 0.281 & 0.543 & 0.012 & 0.144 & 0.002 & 3.74 & 1.37 & 1.00 & 0.73 \\
\hline AudiVWRearCrankPlate & 0.77 & 3.84 & 0.030 & 0.224 & 0.495 & 0.110 & 0.128 & 0.013 & 12.52 & 9.56 & 1.18 & 0.12 \\
\hline BaseBracket & 1.05 & 2.01 & 0.011 & 0.114 & 0.772 & 0.018 & 0.085 & 0.000 & 5.26 & 1.51 & 2.13 & 1.41 \\
\hline
\end{tabular}




\begin{tabular}{|c|c|c|c|c|c|c|c|c|c|c|c|c|}
\hline BearingBody & 1.16 & 2.14 & 0.010 & 0.287 & 0.366 & 0.069 & 0.266 & 0.001 & 7.18 & 0.89 & 0.43 & 0.49 \\
\hline BevelGear & 1.18 & 2.18 & 0.033 & 0.487 & 0.330 & 0.019 & 0.124 & 0.007 & 12.08 & 0.25 & 0.25 & 1.00 \\
\hline BigBlockChevyFlange & 0.63 & 3.77 & 0.122 & 0.231 & 0.287 & 0.318 & 0.043 & 0.000 & 7.30 & 6.34 & 50.69 & 8.00 \\
\hline Block & 1.53 & 3.01 & 0.090 & 0.369 & 0.193 & 0.079 & 0.256 & 0.014 & 13.94 & 1.50 & 1.50 & 1.00 \\
\hline BOVTop2 & 1.19 & 2.78 & 0.040 & 0.234 & 0.392 & 0.071 & 0.249 & 0.013 & 9.83 & 4.75 & 1.04 & 0.22 \\
\hline BRACKET & 1.01 & 4.63 & 0.003 & 0.153 & 0.668 & 0.030 & 0.146 & 0.000 & 7.98 & 3.13 & 0.67 & 0.21 \\
\hline $\begin{array}{c}\text { BRE14LI4NeedleSleeve } \\
\text { BearingBody }\end{array}$ & 1.24 & 5.68 & 0.022 & 0.546 & 0.130 & 0.070 & 0.232 & 0.001 & 27.26 & 1.01 & 0.38 & 0.38 \\
\hline BusBar2Cells40152S & 0.97 & 3.64 & 0.033 & 0.105 & 0.825 & 0.029 & 0.008 & 0.000 & 4.50 & 40.73 & 3.10 & 0.08 \\
\hline CamRetainer & 0.62 & 3.99 & 0.042 & 0.154 & 0.696 & 0.092 & 0.016 & 0.000 & 5.51 & 1.41 & 44.69 & 31.67 \\
\hline camshaft & 0.78 & 2.50 & 0.122 & 0.682 & 0.058 & 0.136 & 0.002 & 0.000 & 5.32 & 0.08 & 1.00 & 12.50 \\
\hline CarburatorBody & 1.34 & 4.68 & 0.055 & 0.384 & 0.141 & 0.072 & 0.318 & 0.029 & 7.34 & 0.49 & 0.48 & 0.99 \\
\hline CastAluminumIntake & 1.35 & 5.07 & 0.106 & 0.308 & 0.128 & 0.069 & 0.331 & 0.058 & 5.72 & 1.47 & 1.52 & 1.03 \\
\hline CastExhaustCollector & 1.47 & 7.15 & 0.099 & 0.352 & 0.006 & 0.035 & 0.502 & 0.006 & 5.46 & 1.41 & 1.07 & 0.76 \\
\hline CetrifugalImpeller & 1.91 & 5.02 & 0.006 & 0.267 & 0.209 & 0.115 & 0.396 & 0.007 & 8.76 & 2.10 & 1.00 & 0.48 \\
\hline ChevyHeaderFlange & 1.08 & 4.21 & 0.030 & 0.281 & 0.264 & 0.127 & 0.287 & 0.011 & 4.70 & 4.67 & 2.10 & 0.45 \\
\hline ClutchCover & 1.19 & 7.02 & 0.013 & 0.238 & 0.301 & 0.222 & 0.202 & 0.024 & 17.60 & 4.00 & 1.00 & 0.25 \\
\hline ClutchPlate1 & 1.44 & 4.48 & 0.013 & 0.421 & 0.395 & 0.037 & 0.125 & 0.008 & 12.95 & 1.00 & 6.00 & 6.00 \\
\hline CollarlinShaft & 1.16 & 2.22 & 0.037 & 0.312 & 0.316 & 0.062 & 0.268 & 0.005 & 11.17 & 1.00 & 3.50 & 3.50 \\
\hline CollarShaft & 0.88 & 2.36 & 0.001 & 0.173 & 0.581 & 0.016 & 0.227 & 0.000 & 6.84 & 6.00 & 1.00 & 0.17 \\
\hline ConnectingRod & 1.08 & 2.71 & 0.045 & 0.322 & 0.357 & 0.062 & 0.204 & 0.011 & 6.17 & 6.07 & 3.64 & 0.60 \\
\hline CoverPlate & 1.11 & 8.51 & 0.012 & 0.136 & 0.799 & 0.011 & 0.042 & 0.000 & 6.84 & 2.36 & 1.33 & 0.56 \\
\hline CrankGear & 1.05 & 5.52 & 0.067 & 0.364 & 0.154 & 0.133 & 0.261 & 0.022 & 26.32 & 10.66 & 1.00 & 0.09 \\
\hline Crankshaft & 0.90 & 3.46 & 0.147 & 0.329 & 0.344 & 0.118 & 0.062 & 0.000 & 11.61 & 0.53 & 0.13 & 0.24 \\
\hline CylinderBlock5cyl & 2.36 & 7.22 & 0.013 & 0.156 & 0.165 & 0.093 & 0.573 & 0.000 & 12.10 & 3.09 & 4.12 & 1.33 \\
\hline Diffuser & 1.36 & 3.87 & 0.033 & 0.271 & 0.405 & 0.071 & 0.193 & 0.027 & 21.33 & 0.30 & 0.30 & 1.00 \\
\hline DISCPLATE & 0.88 & 4.15 & 0.008 & 0.093 & 0.735 & 0.085 & 0.079 & 0.000 & 9.63 & 28.33 & 1.00 & 0.04 \\
\hline ElbowCastIron & 1.34 & 3.35 & 0.080 & 0.236 & 0.413 & 0.080 & 0.153 & 0.039 & 8.08 & 0.87 & 0.86 & 0.98 \\
\hline EngineBlock & 1.78 & 5.14 & 0.033 & 0.317 & 0.215 & 0.057 & 0.364 & 0.013 & 12.21 & 0.72 & 0.68 & 0.95 \\
\hline EngineBottomPlate & 1.14 & 3.64 & 0.014 & 0.230 & 0.574 & 0.034 & 0.145 & 0.004 & 15.18 & 0.10 & 0.20 & 2.00 \\
\hline
\end{tabular}




\begin{tabular}{|c|c|c|c|c|c|c|c|c|c|c|c|c|}
\hline EngineFrontCover & 1.28 & 3.01 & 0.017 & 0.321 & 0.331 & 0.039 & 0.286 & 0.006 & 12.63 & 1.00 & 1.73 & 1.73 \\
\hline EngineGudgeonPin & 1.61 & 3.52 & 0.015 & 0.519 & 0.022 & 0.050 & 0.394 & 0.000 & 4.84 & 3.57 & 3.57 & 1.00 \\
\hline $\begin{array}{c}\text { Ferrule2InchClampTubeO } \\
\text { DwSealGroovePt1 }\end{array}$ & 1.32 & 6.14 & 0.016 & 0.465 & 0.077 & 0.030 & 0.409 & 0.004 & 8.86 & 0.89 & 0.89 & 1.00 \\
\hline $\begin{array}{c}\text { Ferrule2InchClampTubeO } \\
\text { DwSealGroovePt2 }\end{array}$ & 1.08 & 1.64 & 0.024 & 0.374 & 0.515 & 0.029 & 0.056 & 0.001 & 6.23 & 0.33 & 0.33 & 1.00 \\
\hline fitting & 1.40 & 2.93 & 0.051 & 0.432 & 0.132 & 0.040 & 0.345 & 0.000 & 5.42 & 2.71 & 2.35 & 0.87 \\
\hline Flange & 0.85 & 3.81 & 0.001 & 0.075 & 0.722 & 0.024 & 0.171 & 0.007 & 8.66 & 0.60 & 0.67 & 1.13 \\
\hline FlyWheel & 1.59 & 3.53 & 0.038 & 0.346 & 0.246 & 0.056 & 0.276 & 0.039 & 18.84 & 2.34 & 1.00 & 0.43 \\
\hline FordGT40IntakeSpacer & 0.64 & 4.07 & 0.127 & 0.147 & 0.222 & 0.355 & 0.145 & 0.003 & 10.13 & 35.31 & 3.66 & 0.10 \\
\hline FuelPumpDeletePlate & 1.09 & 2.37 & 0.017 & 0.134 & 0.633 & 0.026 & 0.160 & 0.030 & 4.99 & 1.19 & 10.00 & 8.43 \\
\hline gasket & 0.69 & 10.83 & 0.009 & 0.111 & 0.755 & 0.125 & 0.000 & 0.000 & 12.37 & 3.20 & 266.67 & 83.33 \\
\hline Gear & 1.35 & 2.98 & 0.057 & 0.280 & 0.402 & 0.082 & 0.176 & 0.004 & 33.64 & 1.00 & 7.20 & 7.20 \\
\hline GearboxCasing & 1.22 & 6.28 & 0.057 & 0.172 & 0.485 & 0.099 & 0.158 & 0.029 & 8.39 & 1.46 & 8.72 & 5.97 \\
\hline GearBoxTophousing & 1.45 & 3.58 & 0.032 & 0.107 & 0.587 & 0.054 & 0.201 & 0.018 & 15.82 & 1.71 & 4.00 & 2.33 \\
\hline GenevaCam & 0.86 & 2.90 & 0.009 & 0.111 & 0.706 & 0.052 & 0.121 & 0.000 & 7.92 & 0.95 & 13.88 & 14.54 \\
\hline GM3RUJoint & 0.82 & 1.54 & 0.056 & 0.663 & 0.136 & 0.132 & 0.013 & 0.000 & 1.56 & 1.00 & 3.22 & 3.22 \\
\hline GMLS6FrtCover & 1.12 & 6.50 & 0.054 & 0.184 & 0.497 & 0.083 & 0.163 & 0.019 & 7.45 & 1.20 & 6.18 & 5.14 \\
\hline HelicalGearWheel & 1.61 & 4.35 & 0.019 & 0.210 & 0.421 & 0.046 & 0.299 & 0.005 & 34.08 & 1.00 & 3.50 & 3.50 \\
\hline $\begin{array}{c}\text { HondaNSR125Cylinder } \\
\text { HeadGasket } \\
\end{array}$ & 0.61 & 18.16 & 0.007 & 0.301 & 0.451 & 0.049 & 0.191 & 0.001 & 7.92 & 1.07 & 328.54 & 306.94 \\
\hline Housing & 1.65 & 3.78 & 0.022 & 0.272 & 0.202 & 0.111 & 0.354 & 0.039 & 18.09 & 1.00 & 1.34 & 1.34 \\
\hline HousingSquare & 1.38 & 2.91 & 0.003 & 0.063 & 0.487 & 0.052 & 0.382 & 0.013 & 13.20 & 1.09 & 2.47 & 2.27 \\
\hline IgnitionCoilMazda13B & 1.18 & 2.30 & 0.029 & 0.405 & 0.363 & 0.039 & 0.154 & 0.010 & 8.08 & 0.39 & 0.38 & 0.98 \\
\hline Impeller & 1.65 & 3.77 & 0.009 & 0.283 & 0.407 & 0.104 & 0.197 & 0.000 & 11.36 & 1.33 & 1.00 & 0.75 \\
\hline IntakeMobilPart1 & 1.48 & 3.73 & 0.001 & 0.253 & 0.582 & 0.050 & 0.108 & 0.007 & 11.55 & 3.54 & 4.41 & 1.25 \\
\hline IntakePipeComponent-1 & 1.24 & 3.60 & 0.022 & 0.427 & 0.104 & 0.073 & 0.368 & 0.007 & 5.56 & 1.14 & 0.72 & 0.63 \\
\hline IntakeTrumpet & 1.27 & 8.99 & 0.081 & 0.335 & 0.057 & 0.140 & 0.323 & 0.063 & 0.41 & 1.00 & 0.94 & 0.94 \\
\hline JettaActuatorBracket & 0.87 & 3.69 & 0.003 & 0.091 & 0.832 & 0.031 & 0.042 & 0.001 & 6.48 & 1.36 & 1.52 & 1.12 \\
\hline link & 0.87 & 2.76 & 0.029 & 0.196 & 0.606 & 0.051 & 0.118 & 0.000 & 1.70 & 3.14 & 20.21 & 6.43 \\
\hline LockNuts & 1.33 & 3.00 & 0.053 & 0.235 & 0.221 & 0.046 & 0.227 & 0.218 & 9.72 & 1.00 & 1.75 & 1.75 \\
\hline $\begin{array}{c}\text { LowerThermostatHousing } \\
\text { SpacerGM }\end{array}$ & 0.94 & 2.56 & 0.019 & 0.199 & 0.664 & 0.035 & 0.083 & 0.000 & 6.64 & 3.59 & 12.83 & 3.57 \\
\hline
\end{tabular}




\begin{tabular}{|c|c|c|c|c|c|c|c|c|c|c|c|c|}
\hline M20LiftingEyeBolt & 1.06 & 2.30 & 0.377 & 0.150 & 0.031 & 0.433 & 0.007 & 0.003 & 36.22 & 0.59 & 2.39 & 4.03 \\
\hline ManifoldPlate & 0.85 & 3.16 & 0.026 & 0.178 & 0.534 & 0.133 & 0.122 & 0.007 & 7.39 & 4.95 & 27.54 & 5.56 \\
\hline MasterCylinderBracket & 0.89 & 5.85 & 0.004 & 0.099 & 0.837 & 0.030 & 0.029 & 0.001 & 7.48 & 1.46 & 0.99 & 0.68 \\
\hline Miata16IntakeFlange & 0.77 & 3.75 & 0.041 & 0.196 & 0.469 & 0.190 & 0.103 & 0.000 & 9.27 & 3.98 & 36.22 & 9.09 \\
\hline MitsubishiEVOMAPadapter & 1.21 & 2.11 & 0.075 & 0.232 & 0.435 & 0.075 & 0.176 & 0.007 & 8.05 & 2.52 & 2.45 & 0.97 \\
\hline NeedleValveScrew & 1.01 & 2.16 & 0.302 & 0.401 & 0.106 & 0.172 & 0.018 & 0.000 & 13.51 & 0.21 & 1.00 & 4.70 \\
\hline OerlikonBrakeValve & 1.43 & 3.82 & 0.021 & 0.227 & 0.534 & 0.041 & 0.165 & 0.011 & 18.49 & 3.21 & 0.75 & 0.23 \\
\hline $\begin{array}{c}\text { OilCoolerBypasCover } \\
\text { PlateForVW }\end{array}$ & 1.18 & 1.94 & 0.010 & 0.137 & 0.609 & 0.048 & 0.175 & 0.022 & 8.50 & 1.44 & 3.50 & 2.42 \\
\hline OilFilter & 2.16 & 1.61 & 0.060 & 0.592 & 0.227 & 0.052 & 0.055 & 0.014 & 11.58 & 0.79 & 1.00 & 1.26 \\
\hline OilPan & 1.44 & 8.07 & 0.014 & 0.159 & 0.568 & 0.074 & 0.165 & 0.021 & 12.66 & 6.43 & 2.14 & 0.33 \\
\hline OilPanModel2 & 1.23 & 12.92 & 0.038 & 0.232 & 0.507 & 0.039 & 0.160 & 0.024 & 3.62 & 7.35 & 0.55 & 0.07 \\
\hline OldhamCoupling & 1.22 & 1.79 & 0.004 & 0.375 & 0.435 & 0.023 & 0.163 & 0.000 & 5.61 & 1.96 & 1.00 & 0.51 \\
\hline ParametricFlange & 1.19 & 3.99 & 0.003 & 0.271 & 0.454 & 0.050 & 0.219 & 0.004 & 15.48 & 1.99 & 3.18 & 1.60 \\
\hline piston & 1.36 & 2.41 & 0.016 & 0.500 & 0.228 & 0.032 & 0.219 & 0.004 & 17.14 & 1.00 & 0.95 & 0.95 \\
\hline PistonRodPart1 & 0.88 & 2.72 & 0.027 & 0.170 & 0.382 & 0.090 & 0.311 & 0.020 & 7.65 & 0.35 & 4.00 & 11.50 \\
\hline PistonRodPart2 & 0.88 & 2.31 & 0.041 & 0.320 & 0.328 & 0.034 & 0.265 & 0.012 & 6.39 & 1.97 & 3.67 & 1.86 \\
\hline pitch2 & 1.25 & 1.91 & 0.016 & 0.241 & 0.476 & 0.144 & 0.095 & 0.027 & 10.85 & 1.00 & 3.82 & 3.82 \\
\hline PlenumPlate & 0.96 & 5.21 & 0.023 & 0.059 & 0.866 & 0.051 & 0.001 & 0.000 & 4.97 & 63.19 & 2.20 & 0.03 \\
\hline Porsche968Flange & 0.69 & 3.00 & 0.024 & 0.211 & 0.468 & 0.092 & 0.202 & 0.004 & 7.27 & 2.06 & 18.35 & 8.89 \\
\hline Porsche968IntakeFlange & 0.80 & 3.64 & 0.093 & 0.198 & 0.249 & 0.224 & 0.236 & 0.001 & 7.79 & 28.94 & 6.87 & 0.24 \\
\hline PorscheStrutBarTopBrace & 0.97 & 2.61 & 0.004 & 0.112 & 0.845 & 0.023 & 0.015 & 0.000 & 3.05 & 21.79 & 2.14 & 0.10 \\
\hline PressurePlate & 1.40 & 3.03 & 0.024 & 0.295 & 0.364 & 0.062 & 0.252 & 0.003 & 12.14 & 4.00 & 1.00 & 0.25 \\
\hline PulleyForAlternator & 1.78 & 3.73 & 0.080 & 0.287 & 0.287 & 0.051 & 0.286 & 0.009 & 7.45 & 0.85 & 0.85 & 1.00 \\
\hline RockerArm & 0.95 & 2.00 & 0.019 & 0.162 & 0.682 & 0.027 & 0.106 & 0.004 & 5.58 & 0.11 & 0.57 & 5.14 \\
\hline SkidPlateBracket & 0.76 & 5.96 & 0.010 & 0.154 & 0.753 & 0.052 & 0.031 & 0.001 & 6.33 & 0.84 & 1.28 & 1.52 \\
\hline socket & 1.21 & 2.02 & 0.030 & 0.342 & 0.369 & 0.042 & 0.212 & 0.005 & 7.13 & 1.72 & 3.38 & 1.96 \\
\hline SpiralBevelGear & 1.10 & 1.77 & 0.012 & 0.403 & 0.474 & 0.015 & 0.095 & 0.002 & 11.15 & 3.67 & 1.00 & 0.27 \\
\hline SplitPin & 0.79 & 2.74 & 0.122 & 0.795 & 0.004 & 0.079 & 0.000 & 0.000 & 0.21 & 0.04 & 0.22 & 5.58 \\
\hline sproket & 0.99 & 2.80 & 0.043 & 0.130 & 0.464 & 0.122 & 0.240 & 0.000 & 10.23 & 8.38 & 0.98 & 0.12 \\
\hline
\end{tabular}




\begin{tabular}{|c|c|c|c|c|c|c|c|c|c|c|c|c|}
\hline SpurGear & 1.09 & 3.23 & 0.008 & 0.140 & 0.741 & 0.024 & 0.085 & 0.002 & 15.40 & 1.00 & 9.82 & 9.82 \\
\hline SteeringKnuckleFlangeSpacer & 0.53 & 1.51 & 0.006 & 0.213 & 0.702 & 0.031 & 0.044 & 0.003 & 3.25 & 1.03 & 22.07 & 21.33 \\
\hline StraightScrewJoint & 1.36 & 2.20 & 0.029 & 0.385 & 0.282 & 0.050 & 0.248 & 0.006 & 10.28 & 1.37 & 1.58 & 1.15 \\
\hline T3DividedTurboInletFlange & 0.98 & 2.55 & 0.009 & 0.122 & 0.652 & 0.044 & 0.173 & 0.000 & 7.77 & 8.50 & 1.62 & 0.19 \\
\hline ThreeTubeJoint & 0.53 & 2.05 & 0.000 & 0.000 & 0.011 & 0.187 & 0.792 & 0.009 & 0.00 & 1.15 & 5.65 & 4.90 \\
\hline ThrustBearingDisc & 1.03 & 2.90 & 0.026 & 0.204 & 0.541 & 0.088 & 0.113 & 0.027 & 18.59 & 1.17 & 11.82 & 10.10 \\
\hline TowbarElectricsBracket & 0.71 & 6.79 & 0.029 & 0.154 & 0.744 & 0.051 & 0.021 & 0.000 & 4.73 & 0.25 & 2.07 & 8.27 \\
\hline TrussBearing & 1.29 & 2.70 & 0.013 & 0.203 & 0.547 & 0.030 & 0.205 & 0.002 & 8.17 & 3.05 & 2.39 & 0.79 \\
\hline TurboHousing & 1.72 & 6.35 & 0.171 & 0.302 & 0.014 & 0.130 & 0.248 & 0.135 & 7.33 & 2.28 & 1.10 & 0.48 \\
\hline valve & 0.45 & 2.04 & 0.097 & 0.648 & 0.160 & 0.095 & 0.000 & 0.000 & 0.30 & 1.00 & 0.34 & 0.34 \\
\hline ValveClip & 1.02 & 1.98 & 0.010 & 0.489 & 0.177 & 0.103 & 0.219 & 0.003 & 4.87 & 1.23 & 2.00 & 1.62 \\
\hline VWGolfInletFlange & 1.02 & 3.68 & 0.027 & 0.230 & 0.333 & 0.080 & 0.324 & 0.005 & 5.24 & 1.74 & 4.52 & 2.60 \\
\hline W05Lockwasher & 0.57 & 4.48 & 0.035 & 0.207 & 0.669 & 0.030 & 0.057 & 0.001 & 8.02 & 1.00 & 13.40 & 13.35 \\
\hline WheelBearingHousingForAVW & 1.14 & 3.15 & 0.020 & 0.222 & 0.379 & 0.090 & 0.271 & 0.018 & 14.21 & 1.08 & 3.41 & 3.15 \\
\hline WINCHMTGBRK & 0.88 & 5.52 & 0.006 & 0.147 & 0.769 & 0.020 & 0.057 & 0.000 & 6.37 & 1.63 & 1.18 & 0.73 \\
\hline WormGear & 1.08 & 2.16 & 0.130 & 0.545 & 0.201 & 0.090 & 0.033 & 0.001 & 9.00 & 1.00 & 0.21 & 0.21 \\
\hline WormGearDesign & 1.21 & 1.99 & 0.025 & 0.184 & 0.469 & 0.152 & 0.170 & 0.000 & 15.55 & 1.00 & 2.80 & 2.79 \\
\hline WristPin & 1.51 & 3.20 & 0.007 & 0.565 & 0.007 & 0.017 & 0.405 & 0.001 & 2.73 & 1.00 & 0.37 & 0.37 \\
\hline
\end{tabular}




\section{Appendix C: Extended List of Metrics}

Table C1. Potential List of Metrics to Characterize an Object

\begin{tabular}{|c|c|c|c|c|c|c|c|}
\hline $\begin{array}{l}\text { Feature/ } \\
\text { Descriptor }\end{array}$ & $\begin{array}{l}\text { Local or } \\
\text { Global }\end{array}$ & $\begin{array}{l}\text { 2D / 3D } \\
\text { / Both }\end{array}$ & $\begin{array}{l}\text { Shape/Su } \\
\text { rface }\end{array}$ & Description & $\begin{array}{l}\text { Relevant (R) } \\
\text { Non-Relevant } \\
\quad \text { (NR) }\end{array}$ & $\begin{array}{c}\text { Algorithm/ } \\
\text { software } \\
\text { needed } \\
\text { (Y/N), } \\
\text { available? }\end{array}$ & Source \\
\hline Spin images & Local & $3 \mathrm{D}$ & Shape & $\begin{array}{l}\text { Histogram of local point locations given offset from center, } \\
\text { oriented point. }\end{array}$ & $\mathrm{R}$ & Y, available & $\begin{array}{l}\text { Johnson and Hebert } \\
1999\end{array}$ \\
\hline $\begin{array}{l}\text { Intrinsic Spin Image } \\
\text { (ISIs) }\end{array}$ & Local & $3 \mathrm{D}$ & Shape & $\begin{array}{l}\text { A generalization of spin images to use arbitrary subspaces } \\
\text { rather than basic x,y, rectangular coordinate system }\end{array}$ & NR & & \\
\hline SURF & Local & $2 \mathrm{D}$ & $\begin{array}{l}\text { Surface } \\
\text { (texture) }\end{array}$ & $\begin{array}{l}\text { Speeded Up Robust Features (SURF). Local image feature } \\
\text { detector and descriptor based on Haar wavelet response. } \\
\text { Scale/rotation invariant. }\end{array}$ & $\mathrm{R}$ & Y, available & Bay et al., 2006 \\
\hline Color histograms & $\begin{array}{l}\text { Local/ } \\
\text { Global }\end{array}$ & $2 \mathrm{D}$ & Surface & $\begin{array}{l}\text { Histogram of color values in appropriate color space } \\
\text { (RGB, HSB) }\end{array}$ & $\mathrm{R}$ & & $\begin{array}{l}\text { Zivkovic and Krose, } \\
2004\end{array}$ \\
\hline HOG (PHOG) & Local & $2 \mathrm{D}$ & Surface & $\begin{array}{l}\text { Histogram of Oriented Gradients (HOG). Local image } \\
\text { feature descriptor. A histogram of local gradient } \\
\text { orientations computed on subcells in a region, and } \\
\text { normalized by intensity over a larger block. }\end{array}$ & $\mathrm{R}$ & Y, available & $\begin{array}{l}\text { Dalal and Triggs, } \\
2005\end{array}$ \\
\hline Self-similarity & Local & $2 \mathrm{D}$ & Surface & Log-polar histogram descriptor of local point features. & $\mathrm{R}$ & & $\begin{array}{l}\text { Shechtman and Irani, } \\
2007\end{array}$ \\
\hline SIFT & Local & $2 \mathrm{D}$ & Surface & $\begin{array}{l}\text { Scale Invariant Feature Transform (SIFT). Local feature } \\
\text { vector computed from histogram of local gradients and } \\
\text { intensities. }\end{array}$ & $\mathrm{R}$ & Y, available & Lowe, 2004 \\
\hline 2.5D SIFT & Local & & $\begin{array}{l}\text { Shape + } \\
\text { surface }\end{array}$ & Generalization of SIFT to $2.5 \mathrm{D}$ range images. & $\mathrm{R}$ & & Lo and Siebert, 2009 \\
\hline
\end{tabular}




\begin{tabular}{|c|c|c|c|c|c|c|c|}
\hline $\begin{array}{l}\text { Feature/ } \\
\text { Descriptor }\end{array}$ & $\begin{array}{l}\text { Local or } \\
\text { Global }\end{array}$ & $\begin{array}{l}\text { 2D / 3D } \\
\text { / Both }\end{array}$ & $\begin{array}{l}\text { Shape/Su } \\
\text { rface }\end{array}$ & Description & $\begin{array}{l}\text { Relevant (R) } \\
\text { Non-Relevant } \\
\quad \text { (NR) }\end{array}$ & $\begin{array}{c}\text { Algorithm/ } \\
\text { software } \\
\text { needed } \\
\text { (Y/N), } \\
\text { available? }\end{array}$ & Source \\
\hline Shape context & Local & Both & $\begin{array}{l}\text { Shape }+ \\
\text { surface } \\
\text { (pattern) }\end{array}$ & $\begin{array}{l}\text { Shape context. } 2 \mathrm{D} \text { and } 3 \mathrm{D} \text { point feature computed as log- } \\
\text { polar histogram of points, typically along boundaries. Can } \\
\text { be generalized to } 3 \mathrm{D} \text {. }\end{array}$ & $\mathrm{R}$ & Y, available & Malik et al., 2001 \\
\hline LESH & Local & $2 \mathrm{D}$ & Surface & $\begin{array}{l}\text { Local Energy Based Shape Histogram (LESH). Local } \\
\text { image feature descriptor based on 128-dinensional } \\
\text { compact spatial histogram. Scale invariant. }\end{array}$ & NR & & $\begin{array}{l}\text { Sarfraz and } \\
\text { Hellwich, } 2008\end{array}$ \\
\hline MSER & Local & $2 \mathrm{D}$ & Surface & $\begin{array}{l}\text { Maximally Stable Extremal Regions (MSER), A local 2D } \\
\text { image detector and descriptor that uses image morphology } \\
\text { to find blob patterns. }\end{array}$ & NR & Y, available & Matas et al, 2002 \\
\hline GLOH & Local & $2 \mathrm{D}$ & Surface & $\begin{array}{l}\text { Gradient Location and Orientation Histogram (GLOH). } \\
\text { Enhancement of the SIFT operator to use more spatial } \\
\text { regions. }\end{array}$ & NR & & $\begin{array}{l}\text { Mikolajczyk and } \\
\text { Schmid, } 2005\end{array}$ \\
\hline Autocorrelation & Local & $2 \mathrm{D}$ & Surface & $\begin{array}{l}\text { Standard technique for correlating images - power } \\
\text { spectrum will indicate whether image region is distinctive } \\
\text { and suitable for matching. }\end{array}$ & NR & & Deng et. al, 2007 \\
\hline PCBR & Local & $2 \mathrm{D}$ & Surface & $\begin{array}{l}\text { Principal Curvature-Based Region (PCBR) region detector. } \\
\text { A structure based, affine invariant detector that finds } \\
\text { curvature maximums in scale space. }\end{array}$ & NR & & Deng et. al, 2007 \\
\hline Corner detectors & Local & $2 \mathrm{D}$ & Surface & $\begin{array}{l}\text { Many versions (described in review article Mikolajczyk et } \\
\text { al.) All are local image feature detectors that are sensitive } \\
\text { to high curvature, high gradients, and other distinctive } \\
\text { image extrema. }\end{array}$ & $\mathrm{R}$ & Y, available & $\begin{array}{l}\text { Mikolajczyk et al } \\
2005\end{array}$ \\
\hline ART (ang. Radial) & Global & $2 \mathrm{D}$ & Surface & $\begin{array}{l}\text { Angular Radial Transform (ART). Feature descriptor based } \\
\text { on angular decomposition of B\&W image to set of radially } \\
\text { symmetrical basis functions. }\end{array}$ & NR & & Bober, 2001 \\
\hline RIFT & Local & $2 \mathrm{D}$ & Surface & $\begin{array}{l}\text { Rotation-Invariant Feature Transform (RIFT). Rotational } \\
\text { invariant version of SIFT detector. }\end{array}$ & NR & & Lazebnik et al, 2005 \\
\hline
\end{tabular}




\begin{tabular}{|c|c|c|c|c|c|c|c|}
\hline $\begin{array}{l}\text { Feature/ } \\
\text { Descriptor }\end{array}$ & $\begin{array}{l}\text { Local or } \\
\text { Global }\end{array}$ & $\begin{array}{l}\text { 2D / 3D } \\
\text { / Both }\end{array}$ & $\begin{array}{l}\text { Shape/Su } \\
\text { rface }\end{array}$ & Description & $\begin{array}{l}\text { Relevant (R) } \\
\text { Non-Relevant } \\
\quad \text { (NR) }\end{array}$ & $\begin{array}{l}\text { Algorithm/ } \\
\text { software } \\
\text { needed } \\
\text { (Y/N), } \\
\text { available? }\end{array}$ & Source \\
\hline Shock Scaffold & Global & $3 \mathrm{D}$ & Shape & $\begin{array}{l}\text { The shock scaffold is a hierarchical organization of the } \\
\text { medial axis in 3D consisting of special medial points, and } \\
\text { curves connecting these points, thereby forming a } \\
\text { geometric directed graph }\end{array}$ & $\mathrm{R}$ & & $\begin{array}{l}\text { Laymarie and Kimia, } \\
2001\end{array}$ \\
\hline $\begin{array}{l}\text { 3D Zernike } \\
\text { Descriptors }\end{array}$ & Global & $3 \mathrm{D}$ & Shape & $\begin{array}{l}\text { A vector of } 3 D \text { shape descriptors based on affine invariant } \\
\text { moments of } 3 D \text { point cloud based on Zernike polynomials, } \\
\text { which are orthogonal on the unit sphere. }\end{array}$ & $\mathrm{R}$ & Y, available & $\begin{array}{l}\text { Novotni and Klein, } \\
2003\end{array}$ \\
\hline $\begin{array}{l}\text { Radius-based } \\
\text { Surface Descriptor } \\
\text { (RSD) }\end{array}$ & Global & $3 \mathrm{D}$ & Shape & $\begin{array}{l}\text { Symmetric model fitting from point cloud data, using } \\
\text { sample consensus to fit generalized cylinders (the } \\
\text { approximate radius of the smallest fitting curve to a local } \\
\text { neighborhood.) }\end{array}$ & $\mathrm{R}$ & $\begin{array}{c}\text { Yes, in } \\
\text { ROS/PCL }\end{array}$ & Marton et al, 2010 \\
\hline $\begin{array}{l}\text { PC (Principal } \\
\text { Curvature) }\end{array}$ & Local & $3 \mathrm{D}$ & Shape & $\begin{array}{l}\text { Local shape descriptor of principle curvature of local } \\
\text { points. Very general descriptor, generally not useful by } \\
\text { itself. }\end{array}$ & $\mathrm{R}$ & & $\begin{array}{l}\text { Rusu and Cousins, } \\
2011\end{array}$ \\
\hline $\begin{array}{l}\text { FPFH (Fast Pt. Ft. } \\
\text { Hist.) }\end{array}$ & Local & $3 \mathrm{D}$ & Shape & $\begin{array}{l}6 \mathrm{D} \text { pose invariant local shape descriptor that generalizes } \\
\text { mean curvature by encoding neighborhood properties in } \\
\text { histogram of local normal differential features. }\end{array}$ & NR & & $\begin{array}{l}\text { Rusu, Blodow, } \\
\text { Beetz, } 2009\end{array}$ \\
\hline Global FPFH & Global & $3 \mathrm{D}$ & Shape & $\begin{array}{l}\text { A global shape descriptor that uses the FPFH } \\
\text { classification of local nodes in the creation of pairwise } \\
\text { histograms between feature points. A ray between each } \\
\text { pair of ft. pts. is intersected with nodes and a histogram } \\
\text { generated for that pair of the classes of intersecting nodes. }\end{array}$ & NR & & $\begin{array}{l}\text { Rusu, Hozbach, } \\
\text { Beetz, Bradski, } \\
2009\end{array}$ \\
\hline 3D Shape Context & Local & $3 \mathrm{D}$ & Shape & $\begin{array}{l}\text { A local shape descriptor computed by a spherically } \\
\text { indexed histogram of local point position around a given } \\
\text { point, the north pole given by the surface normal at the } \\
\text { given point. }\end{array}$ & $\mathrm{R}$ & & Tombari et al, 2010 \\
\hline $\begin{array}{l}\text { VFH (Viewpt. Ft. } \\
\text { Hist.) }\end{array}$ & Local & $3 \mathrm{D}$ & Shape & $\begin{array}{l}\text { A local shape descriptor based on the FPFH that integrates } \\
\text { the viewpoint normal into the histogram calculations. }\end{array}$ & $\mathrm{R}$ & & $\begin{array}{l}\text { Rusu, Bradski, } \\
\text { Thibaux, Hsu, } 2010\end{array}$ \\
\hline Point Signature & Local & $3 \mathrm{D}$ & Shape & $\begin{array}{l}\text { Point descriptor [Chua and Jarvis [CJ97]] used a similar } \\
\text { sampling, but recorded the distance from the contour to a } \\
\text { plane fit to the contour and passing through the point. }\end{array}$ & $\mathrm{R}$ & & $\begin{array}{l}\text { Chua and Jarvis, } \\
1997\end{array}$ \\
\hline
\end{tabular}




\begin{tabular}{|c|c|c|c|c|c|c|c|}
\hline $\begin{array}{l}\text { Feature/ } \\
\text { Descriptor }\end{array}$ & $\begin{array}{l}\text { Local or } \\
\text { Global }\end{array}$ & $\begin{array}{l}\text { 2D / 3D } \\
\text { / Both }\end{array}$ & $\begin{array}{c}\text { Shape/Su } \\
\text { rface }\end{array}$ & Description & $\begin{array}{l}\text { Relevant (R) } \\
\text { Non-Relevant } \\
\quad \text { (NR) }\end{array}$ & $\begin{array}{l}\text { Algorithm/ } \\
\text { software } \\
\text { needed } \\
\text { (Y/N), } \\
\text { available? }\end{array}$ & Source \\
\hline $\begin{array}{l}\text { Intrinsic Shape } \\
\text { Signatures }\end{array}$ & Local & $3 \mathrm{D}$ & Shape & $\begin{array}{l}\text { Local 3D measure based on local support compute the } \\
\text { Eigen Value Decomposition (EVD) of the scatter matrix of } \\
\text { the points belonging to the support. ISS uses as } \\
\text { distinctiveness the magnitude of the smallest eigenvalue } \\
\text { and the ratio between two successive eigenvalues. }\end{array}$ & $\mathrm{R}$ & & Zhong, 2009 \\
\hline $\begin{array}{l}\text { Heat Kernel } \\
\text { Signatures }\end{array}$ & Local & $3 \mathrm{D}$ & Shape & $\begin{array}{l}\text { Local measure based on eigenvalue decomposition of heat } \\
\text { kernel. Invariant to isometric transformation. Represents } \\
\text { decomposition into eigenvalues and eigenfunctions of } \\
\text { Laplace-Beltrami operator. }\end{array}$ & $\mathrm{R}$ & Y, available & $\begin{array}{l}\text { Bronstein and } \\
\text { Kokkinos, } 2010\end{array}$ \\
\hline GPS & Global & $3 \mathrm{D}$ & Shape & $\begin{array}{l}\text { A deformation invariant representation of surfaces, the } \\
\text { GPS embedding, is introduced using the eigenvalues and } \\
\text { eigenfunctions of the Laplace-Beltrami differential } \\
\text { operator. It is based on objects of global character, the } \\
\text { obtained representation is robust to local topology changes. } \\
\text { The GPS embedding captures enough information to } \\
\text { handle various shape processing tasks as shape } \\
\text { classification, segmentation, and correspondence. }\end{array}$ & $\mathrm{R}$ & & Rustamov, 2007 \\
\hline $\begin{array}{l}\text { CVFH (Clustered } \\
\text { ViewPt. Ft. Hist. }\end{array}$ & $\begin{array}{l}\text { Semi- } \\
\text { global }\end{array}$ & $3 \mathrm{D}$ & Shape & $\begin{array}{l}\text { Local 3D shape descriptor of stable regions using a local } \\
\text { reference frame, building a histogram of angles to other } \\
\text { points, including Shape Distribution Component (SDC). }\end{array}$ & $\mathrm{R}$ & & Aldoma et al, 2012 \\
\hline NARF & Local & $3 \mathrm{D}$ & Shape & $\begin{array}{l}\text { Normal Aligned Radial Feature is a local 3D shape } \\
\text { detector and descriptor for point cloud data, a histogram of } \\
\text { curvature changes along radial directors. }\end{array}$ & $\mathrm{R}$ & & Steder et al., 2010 \\
\hline HK Segmentation & Local & $3 \mathrm{D}$ & Shape & $\begin{array}{l}\text { Gaussian }(\mathrm{K}) \text { and mean }(\mathrm{H}) \text { curvature measures computed } \\
\text { for each local point. }\end{array}$ & $\mathrm{R}$ & Y, available & Besl and Jain, 1986 \\
\hline SC Shape Index & Local & $3 \mathrm{D}$ & Shape & $\begin{array}{l}\text { A revised version of } \mathrm{H} \text { and } \mathrm{K} \text { that decouples the shape } \\
\text { characteristic (nature of curvature) and shape strength } \\
\text { (magnitude of curvature.) }\end{array}$ & $\mathrm{R}$ & Y, available & $\begin{array}{l}\text { Koenderink and van } \\
\text { Doorn, } 1992\end{array}$ \\
\hline $\begin{array}{l}\text { Degree of } \\
\text { symmetry/Bilateral } \\
\text { or radial symmetry }\end{array}$ & Global & Both & Shape & Some number which shows how symmetric an object is. & NR & & \\
\hline
\end{tabular}




\begin{tabular}{|c|c|c|c|c|c|c|c|}
\hline $\begin{array}{l}\text { Feature/ } \\
\text { Descriptor }\end{array}$ & $\begin{array}{l}\text { Local or } \\
\text { Global }\end{array}$ & $\begin{array}{c}\text { 2D / 3D } \\
\text { / Both }\end{array}$ & $\begin{array}{c}\text { Shape/Su } \\
\text { rface }\end{array}$ & Description & $\begin{array}{l}\text { Relevant (R) } \\
\text { Non-Relevant } \\
\quad \text { (NR) }\end{array}$ & $\begin{array}{l}\text { Algorithm/ } \\
\text { software } \\
\text { needed } \\
\text { (Y/N), } \\
\text { available? }\end{array}$ & Source \\
\hline Chirality & Global & Both & Shape & $\begin{array}{l}\text { Chirality is a property of asymmetry important in several } \\
\text { branches of science. } \\
\text { An object or a system is chiral if it is not identical to its } \\
\text { mirror image, that is, it cannot be superposed onto it. }\end{array}$ & NR & & \\
\hline Compactness & Global & Both & Shape & $\begin{array}{l}\text { The compactness measure of a shape, sometimes called } \\
\text { the shape factor, is a numerical quantity representing the } \\
\text { degree to which a shape is compact } \\
\text { The compactness measure of a shape, sometimes called the } \\
\text { shape factor, is a numerical quantity representing the } \\
\text { degree to which a shape is compact }\end{array}$ & $\mathrm{R}$ & Y, available & MacEachren, 1985 \\
\hline Convexity & Global & Both & Shape & $\begin{array}{l}\text { Definition } 1 \text {. For a given 3D closed mesh M, where the } \\
\text { convex hull of } \mathrm{M} \text { is } \mathrm{CH}(\mathrm{M}) \text {, we define its convexity } \\
\text { measure } \mathrm{C} 1(\mathrm{M}) \text { as } \\
\mathrm{C} 1(\mathrm{M})=\text { Volume }(\mathrm{M}) / \mathrm{Volume}(\mathrm{CH}(\mathrm{M})) \\
\text { Definition } 2 \text {. For a given 3D closed mesh M, where the } \\
\text { convex hull of M is CH(M), we define its convexity } \\
\text { measure C2(M) as } \\
\mathrm{C} 2(\mathrm{M})=\text { SurfaceArea(M) / SurfaceArea }(\mathrm{CH}(\mathrm{M}))\end{array}$ & $\mathrm{R}$ & Y, available & $\begin{array}{l}\text { Žunić, J. and Rosin, } \\
\text { P. L. , } 2004\end{array}$ \\
\hline Ellipticity & Global & Both & Shape & How elliptical is the $3 \mathrm{D}$ model? & NR & & $\begin{array}{l}\text { Aktaş and Žunić, } \\
2011\end{array}$ \\
\hline Rectangularity & Global & Both & Shape & How rectangular is the $3 \mathrm{D}$ model? & NR & & Rosin, 2003 \\
\hline Rectilinearity & Global & Both & Shape & How rectilinear is the $3 \mathrm{D}$ model? & NR & & $\begin{array}{l}\text { Žunić, J. and Rosin, } \\
\text { P. L., } 2003\end{array}$ \\
\hline Triangularity & Global & Both & Shape & How triangular is the $3 \mathrm{D}$ model & NR & & Rosin, 2003 \\
\hline Roundness & Global & $3 \mathrm{D}$ & Shape & $\begin{array}{l}\text { Roundness is the degree of smoothing due to abrasion of } \\
\text { sedimentary particles. It is expressed as the ratio of the } \\
\text { average radius of curvature of the edges or corners to the } \\
\text { radius of curvature of the maximum inscribed sphere. }\end{array}$ & $\mathrm{R}$ & Y, available & Wadell, 1932 \\
\hline
\end{tabular}




\begin{tabular}{|c|c|c|c|c|c|c|c|}
\hline $\begin{array}{l}\text { Feature/ } \\
\text { Descriptor }\end{array}$ & $\begin{array}{l}\text { Local or } \\
\text { Global }\end{array}$ & $\begin{array}{c}\text { 2D / 3D } \\
\text { / Both }\end{array}$ & $\begin{array}{l}\text { Shape/Su } \\
\text { rface }\end{array}$ & Description & $\begin{array}{l}\text { Relevant (R) } \\
\text { Non-Relevant } \\
\quad \text { (NR) }\end{array}$ & $\begin{array}{c}\text { Algorithm/ } \\
\text { software } \\
\text { needed } \\
\text { (Y/N), } \\
\text { available? } \\
\end{array}$ & Source \\
\hline Sphericity & Global & $3 \mathrm{D}$ & Shape & $\begin{array}{l}\text { Sphericity is a measure of how spherical (round) an object } \\
\text { is. As such, it is a specific example of a compactness } \\
\text { measure of a shape. }\end{array}$ & NR & Y, available & $\begin{array}{l}\text { Mora and Kwan, } \\
2000\end{array}$ \\
\hline Volume & Global & $3 \mathrm{D}$ & Shape & Volume of model & $\mathrm{R}$ & Y, available & Corney et al., 2002 \\
\hline Surface area & Global & $3 \mathrm{D}$ & Shape & Surface area of model & $\mathrm{R}$ & Y, available & Corney et al., 2002 \\
\hline $\begin{array}{l}\text { Volume/Surface } \\
\text { Area }\end{array}$ & Global & $3 \mathrm{D}$ & Shape & Volume/Surface Area of model & $\mathrm{R}$ & Y, available & Corney et al., 2002 \\
\hline $\begin{array}{l}\text { Bounding box } \\
\text { aspect ratio }\end{array}$ & Global & $3 \mathrm{D}$ & Shape & Ratio of the longest edge to the shortest edge. & $\mathrm{R}$ & Y, available & Corney et al., 2002 \\
\hline Crinkliness & Global & $3 \mathrm{D}$ & Shape & $\begin{array}{l}\text { Defined as the surface area of the model divided by the } \\
\text { surface area of a sphere having the same volume as the } \\
\text { model. }\end{array}$ & NR & & Jayanti et al., 2006 \\
\hline Hull compactness & Global & $3 \mathrm{D}$ & Shape & $\begin{array}{l}\text { Non-dimensional ratio of convex hull's surface area cubed } \\
\text { to volume of convex hull squared }\end{array}$ & NR & & Corney et al., 2002 \\
\hline Number of facets & Global & $3 \mathrm{D}$ & Shape & Number of facets on the model & NR & & Corney et al., 2002 \\
\hline Number of holes & Global & $3 \mathrm{D}$ & Shape & No of holes in a 3D model & $\mathrm{R}$ & & Corney et al., 2002 \\
\hline Hull crumpliness & Global & $3 \mathrm{D}$ & Shape & $\begin{array}{l}\text { Ratio of object surface area to surface area of its convex } \\
\text { hull }\end{array}$ & NR & & Corney et al., 2002 \\
\hline Hull packing & Global & $3 \mathrm{D}$ & Shape & $\begin{array}{l}\% \text { of convex hull volume not occupied by original object } \\
\text { and is equal to: } \\
\qquad 1-\frac{V_{\text {model }}}{V_{\text {hull }}}\end{array}$ & NR & & Corney et al., 2002 \\
\hline D1 & Global & $3 \mathrm{D}$ & Shape & Histogram of radius & $\mathrm{R}$ & Y, available & $\begin{array}{l}\text { Osada, R., } \\
\text { Funkhouser, T., } \\
\text { Chazelle, B. and } \\
\text { Dobkin, D }\end{array}$ \\
\hline
\end{tabular}




\begin{tabular}{|c|c|c|c|c|c|c|c|}
\hline $\begin{array}{l}\text { Feature/ } \\
\text { Descriptor }\end{array}$ & $\begin{array}{l}\text { Local or } \\
\text { Global }\end{array}$ & $\begin{array}{l}\text { 2D / 3D } \\
\text { / Both }\end{array}$ & $\begin{array}{l}\text { Shape/Su } \\
\text { rface }\end{array}$ & Description & $\begin{array}{l}\text { Relevant (R) } \\
\text { Non-Relevant } \\
\quad \text { (NR) }\end{array}$ & $\begin{array}{c}\text { Algorithm/ } \\
\text { software } \\
\text { needed } \\
\text { (Y/N), } \\
\text { available? } \\
\end{array}$ & Source \\
\hline $\begin{array}{l}\text { D2 Shape } \\
\text { distribution }\end{array}$ & Global & $3 \mathrm{D}$ & Shape & $\begin{array}{l}\text { A histogram of distances between pairs of points on the } \\
\text { surface }\end{array}$ & $\mathrm{R}$ & Y, available & $\begin{array}{l}\text { Osada, } 2001 \text { (in } \\
\text { Shilane et al., 2006) }\end{array}$ \\
\hline Moments & Global & $3 \mathrm{D}$ & Shape & Moment (Hu, Zernike, etc) of models & $\mathrm{R}$ & Y, available & $\begin{array}{l}\text { Novotni and Klein, } \\
2003\end{array}$ \\
\hline $\begin{array}{l}\text { Extended Gaussian } \\
\text { images (EGI) }\end{array}$ & Global & $3 \mathrm{D}$ & Shape & $\begin{array}{l}\text { Spherical function giving the distribution of surface } \\
\text { normals }\end{array}$ & $\mathrm{R}$ & & $\begin{array}{l}\text { Horn, } 1984 \text { (in } \\
\text { Shilane et al., 2006) }\end{array}$ \\
\hline $\begin{array}{l}\text { Shape histograms - } \\
\text { SHELLS }\end{array}$ & Global & $3 \mathrm{D}$ & Shape & $\begin{array}{l}\text { Histogram of distances from the center of mass to points } \\
\text { on the surface }\end{array}$ & $\mathrm{R}$ & & $\begin{array}{l}\text { Ankerst et al., } 1999 \\
\text { (in Shilane et al., } \\
\text { 2006) }\end{array}$ \\
\hline $\begin{array}{l}\text { Shape historgrams - } \\
\text { SECTORS }\end{array}$ & Global & $3 \mathrm{D}$ & Shape & $\begin{array}{l}\text { A spherical function giving the distribution of model area } \\
\text { as a function of spherical angle }\end{array}$ & $\mathrm{R}$ & & $\begin{array}{l}\text { Ankerst et al., } 1999 \\
\text { (in Shilane et al., } \\
\text { 2006) }\end{array}$ \\
\hline $\begin{array}{l}\text { Spherical harmonic } \\
\text { descriptor (SHD) }\end{array}$ & Global & $3 \mathrm{D}$ & Shape & $\begin{array}{l}\text { A rotation invariant representation of the GEDT obtained } \\
\text { by computing the restriction of the function to concentric } \\
\text { spheres and storing the norm of each (harmonic) frequency }\end{array}$ & $\mathrm{R}$ & Y, available & $\begin{array}{l}\text { Kazhdan et al. } 2003 \\
\text { (in Shilane et al., } \\
\text { 2006) }\end{array}$ \\
\hline $\begin{array}{l}\text { Spherical extent } \\
\text { function }(\mathrm{EXT})\end{array}$ & Global & $3 \mathrm{D}$ & Shape & $\begin{array}{l}\text { A spherical function giving the maximal distance from } \\
\text { center of mass } \\
\text { as a function of spherical angle }\end{array}$ & $\mathrm{R}$ & & $\begin{array}{l}\text { Saupe and Vranic, } \\
2001 \text { (in Shilane et } \\
\text { al., 2006) }\end{array}$ \\
\hline $\begin{array}{l}\text { Radialized spherical } \\
\text { extent function } \\
\text { (REXT) }\end{array}$ & Global & $3 \mathrm{D}$ & Shape & $\begin{array}{l}\text { A collection of spherical functions giving the maximal } \\
\text { distance from center of mass as a function of spherical } \\
\text { angle and radius }\end{array}$ & $\mathrm{R}$ & & $\begin{array}{l}\text { Vranic } 2003 \text { (in } \\
\text { Shilane et al., 2006) }\end{array}$ \\
\hline $\begin{array}{l}\text { 3D Wavelet } \\
\text { descriptor }\end{array}$ & Global & $3 \mathrm{D}$ & Shape & $\begin{array}{l}\text { They propose the usage of spherical wavelet transform as } \\
\text { a tool for the analysis of 3D shapes represented by } \\
\text { functions on the unit sphere. We introduce three new shape } \\
\text { descriptors extracted from the spherical wavelet } \\
\text { coefficients, namely: (1) a subset of the spherical wavelet } \\
\text { coefficients, (2) the L1 and, (3) the L2 energies of the } \\
\text { spherical wavelet sub-bands. }\end{array}$ & $\mathrm{R}$ & & Laga H. et al., 2006 \\
\hline Skeleton descriptor & Global & $3 \mathrm{D}$ & Shape & Skeleton descriptor & $\mathrm{R}$ & & Li, Chunyuan, 2013 \\
\hline $\begin{array}{l}\text { Reeb graph } \\
\text { descriptor }\end{array}$ & Global & $3 \mathrm{D}$ & Shape & Skeleton descriptor & $\mathrm{R}$ & & Li, Chunyuan, 2013 \\
\hline
\end{tabular}




\begin{tabular}{|c|c|c|c|c|c|c|c|}
\hline $\begin{array}{l}\text { Feature/ } \\
\text { Descriptor }\end{array}$ & $\begin{array}{l}\text { Local or } \\
\text { Global }\end{array}$ & $\begin{array}{l}\text { 2D / 3D } \\
\text { / Both }\end{array}$ & $\begin{array}{l}\text { Shape/Su } \\
\text { rface }\end{array}$ & Description & $\begin{array}{l}\text { Relevant (R) } \\
\text { Non-Relevant } \\
\quad \text { (NR) }\end{array}$ & $\begin{array}{l}\text { Algorithm/ } \\
\text { software } \\
\text { needed } \\
\text { (Y/N), } \\
\text { available? }\end{array}$ & Source \\
\hline $\begin{array}{l}\text { Light field } \\
\text { descriptor (LFD) - } \\
\text { View base }\end{array}$ & Global & $3 \mathrm{D}$ & Shape & $\begin{array}{l}\text { A representation of a model as a collection of images } \\
\text { rendered from uniformly sampled positions on a view } \\
\text { sphere. The distance between two descriptors is defined as } \\
\text { the minimum L1-difference, taken over all rotations and } \\
\text { all pairings of vertices on two dodecahedra. }\end{array}$ & $\mathrm{R}$ & Y, available & $\begin{array}{l}\text { Chen et al., } 2003 \text { (in } \\
\text { Shilane et al., 2006) } \\
{[10]}\end{array}$ \\
\hline DESIRE & Global & $3 \mathrm{D}$ & Shape & $\begin{array}{l}\text { Composite 3D-shape feature vector (DESIRE), which is } \\
\text { formed using depth buffer images, silhouettes, and ray- } \\
\text { extents of a polygonal mesh. }\end{array}$ & $\mathrm{R}$ & Y, available & Vranic, D. V \\
\hline $\begin{array}{l}\text { View based with 2D } \\
\text { Polar-Fourier } \\
\text { coefficients, Zernike } \\
\text { moments and } \\
\text { Krawtchouk } \\
\text { moments, 2D } \\
\text { Discrete Wavelet } \\
\text { coefficients }\end{array}$ & Global & $3 \mathrm{D}$ & Shape & $\begin{array}{l}\text { View based method, based on Polar-Fourier coefficients, } \\
\text { Zernike moments and Krawtchouk moments, 2D Discrete } \\
\text { Wavelet coefficients }\end{array}$ & $\mathrm{R}$ & & $\begin{array}{l}\text { Daras and } \\
\text { Axenopoulos (2009) } \\
\text { and } \\
\text { Papadakis,, et al., } \\
2010\end{array}$ \\
\hline $\begin{array}{l}\text { Convex Hull } \\
\text { Histogram }(\mathrm{CHH})\end{array}$ & Global & $3 \mathrm{D}$ & Shape & $\begin{array}{l}\text { Compute the 3D convex hull for a given model using the } \\
\text { Quickhull [Barber et al., 1996] algorithm. Then build a } \\
\text { histogram of the pairwise distances based on the points } \\
\text { obtained from the convex hull [Boutin and Kemper, 2004, } \\
\text { Kalyanaraman et al. 2005]. This method is identical to the } \\
\text { basic idea described in [Rea et al, 2005] which presents a } \\
\text { detailed study analyzing the merits of this method. }\end{array}$ & $\mathrm{R}$ & & Jayanti et al., 2006 \\
\hline $\begin{array}{l}\text { 3D shape } \\
\text { histogram-Solid } \\
\text { Angle Histogram }\end{array}$ & Global & $3 \mathrm{D}$ & Shape & $\begin{array}{l}\text { A histogram based on the Solid Angle between random } \\
\text { points }\end{array}$ & $\mathrm{R}$ & & $\begin{array}{l}\text { Ankerst, M., et al. } \\
1999\end{array}$ \\
\hline $\begin{array}{l}\text { Similarity metrics ( } \\
\text { L1, L2, etc), Bag of } \\
\text { words approach, } \\
\text { Sparse coding, } \\
\text { classifier, etc }\end{array}$ & & Both & Both & General purpose tools & $\mathrm{R}$ & Y, available & $\begin{array}{l}\text { Ohbuchi, et. al., } \\
2008\end{array}$ \\
\hline $\begin{array}{l}\text { Harmonic shape } \\
\text { context }\end{array}$ & Local & $3 \mathrm{D}$ & Shape & $\begin{array}{l}\text { Harmonic shape contexts (HSC) local features are } \\
\text { invariant to translation, scale, and 3D rotation. }\end{array}$ & $\mathrm{R}$ & Y, available & Frome, et al., 2007 \\
\hline
\end{tabular}




\begin{tabular}{|c|c|c|c|c|c|c|c|}
\hline $\begin{array}{l}\text { Feature/ } \\
\text { Descriptor }\end{array}$ & $\begin{array}{l}\text { Local or } \\
\text { Global }\end{array}$ & $\begin{array}{l}\text { 2D / 3D } \\
\text { / Both }\end{array}$ & $\begin{array}{l}\text { Shape/Su } \\
\text { rface }\end{array}$ & Description & $\begin{array}{l}\text { Relevant (R) } \\
\text { Non-Relevant } \\
\quad \text { (NR) }\end{array}$ & $\begin{array}{l}\text { Algorithm/ } \\
\text { software } \\
\text { needed } \\
\text { (Y/N), } \\
\text { available? }\end{array}$ & Source \\
\hline $\begin{array}{l}\text { Blowing bubbles } \\
\text { (L1) }\end{array}$ & Local & $3 \mathrm{D}$ & Shape & $\begin{array}{l}\text { Blowing bubbles intersects the surface with a set of } \\
\text { concentric spheres and extracts information about the } \\
\text { surface in two steps. First, they simply count the number of } \\
\text { closed contours, ignoring curves that are far away. }\end{array}$ & NR & Y, available & Heider, et al., 2011 \\
\hline Geodesic fans (L5) & Local & $3 \mathrm{D}$ & Shape & $\begin{array}{l}\text { Geodesic fans sample a metric (such as curvature) on the } \\
\text { mesh using concentric geodesic rings instead of spheres. } \\
\text { Geodesic fans re-sample the metric into evenly spaced } \\
\text { samples in the radial and angular direction. }\end{array}$ & NR & Y, available & Heider, et al., 2011 \\
\hline $\begin{array}{l}\text { Splash descriptor } \\
\text { (L2) }\end{array}$ & Local & $3 \mathrm{D}$ & Shape & $\begin{array}{l}\text { 3D mesh descriptor that computes normal information } \\
\text { along } 3 \mathrm{D} \text { curves of maximum curvature and converts to } 2 \mathrm{D} \\
\text { curve that encodes local curvature and torsion }\end{array}$ & NR & & $\begin{array}{l}\text { Stein and Medioni, } \\
1992\end{array}$ \\
\hline Point descriptor (17) & Local & $3 \mathrm{D}$ & Shape & $\begin{array}{l}\text { Point descriptor [Chua and Jarvis] used a similar } \\
\text { sampling, but recorded the distance from the contour to a } \\
\text { plane fit to the contour and passing through the point }\end{array}$ & NR & Y, available & Chua and Jarvis \\
\hline Normal Distribution & Local & $3 \mathrm{D}$ & Shape & It is a local feature based on the normal distribution. & $\mathrm{R}$ & Y, available & Heider, et al., 2011 \\
\hline $\begin{array}{l}\text { Curvatures: mainly } \\
\text { four curvature } \\
\text { values, Mean, } \\
\text { Gaussian, Shape and } \\
\text { Curvature index (SI, } \\
\text { CI) [L2] }\end{array}$ & Local & $3 \mathrm{D}$ & Shape & $\begin{array}{l}\text { Based on the Curvatures values the following features are } \\
\text { calculated: Mean, Gaussian, Shape and Curvature index. }\end{array}$ & $\mathrm{R}$ & Y, available & Heider, et al., 2011 \\
\hline $\begin{array}{l}\text { Geodesics, heat and } \\
\text { diffusion }\end{array}$ & Local & $3 \mathrm{D}$ & Shape & $\begin{array}{l}\text { Geodesic and Heat diffusion: The techniques in this class } \\
\text { do not directly measure the geodesics, but instead measure } \\
\text { a diffusion process flowing along the geodesics. }\end{array}$ & $\mathrm{R}$ & Y, available & Bronstein, 2011 \\
\hline
\end{tabular}




\section{References for Table C1}

Aktaş, M. A., and Žunić, J., Measuring shape ellipticity, In Computer Analysis of Images and Patterns, Springer Berlin Heidelberg, 2011, pp. 170-177.

Aldoma, A., Tombari, F., Rusu. R.B., and Vincze, M., OUR-CVFH - Oriented, Unique and Repeatable Clustered Viewpoint Feature Histogram for Object Recognition and 6DOF Pose Estimation, Pattern Recognition: Lecture Notes in Computer Science, 7476, 2012, pp 113-122

Ankerst, M., Kastenmüller, G., Kriegel, H.-P., and Seidl, T., Nearest neighbor classification in 3D protein databases, in Proc. ISMB, 1999.

Barber, B. C., Dobkin, D., Huhdanpaa, H., The Quickhull algorithm for convex hulls, ACM Transactions on Mathematical Software 1996;22 (4):469-83.

Bay, H., Tuytelaars, T., and Van Gool, L., "Surf: Speeded up robust features," Computer Vision-ECCV 2006. Springer Berlin Heidelberg, 2006, pp. 404-417.

Besl, P. J., and Jain, R., Segmentation through symbolic surface descriptions, in Proc. IEEE Conf. Computer Vision and Pattern Recognition, June, 1986, pp. 77-85.

Bober, M., MPEG-7 visual shape descriptors, Circuits and Systems for Video Technology, IEEE Transactions on 11.6, 2001, pp. 716-719.

Boutin, M. and Kemper, G., On graph matching, PRECISE technical report, PRE-TR-2004-1, West Lafayette (IN): Purdue University; 2004.

Bronstein, A. M., Bronstein, M. M., Guibas, L. J., and Ovsjanikov, M., Shape google: Geometric words and expressions for invariant shape retrieval, ACM Transactions on Graphics (TOG) 30, no. 1 (2011): 1.

Bronstein, M. M., and Kokkinos, I., Scale-invariant heat kernel signatures for non-rigid shape recognition, Computer Vision and Pattern Recognition (CVPR), 2010 IEEE Conference on. IEEE, 2010.

Chen, D.-Y., Ouhyoung, M., Tian, X.-P. and Shen, Y.-T., On visual similarity based 3D model retrieval, Computer Graphics Forum, 2003, pp. 223-232.

Chua, C. S., and Jarvis, R., Point signatures: A new representation for 3d object recognition, International Journal of Computer Vision, 25.1, 1997, pp. 63-85.

Corney J, Rea H, Clark D, Pritchard J, Breaks M, Macleod R (2002), Coarse filters for shape matching, IEEE Comput Graph Appl 22(3):65-74.

Dalal, N., and Triggs, B., Histograms of oriented gradients for human detection, Computer Vision and Pattern Recognition, 2005. CVPR 2005. IEEE Computer Society Conference on. Vol. 1. IEEE, 2005.

Daras, P. and Axenopoulos, A., "A compact multi-view descriptor for 3D object retrieval." Content-Based Multimedia Indexing, 2009. CBMI'09. Seventh International Workshop on. IEEE, 2009.

Deng, H., Zhang, W., Mortensen, E., Diettrich, T., and Shapiro, L., Principal curvature-based region detector for object recognition, Computer Vision and Pattern Recognition, 2007. CVPR'07. IEEE Conference on. IEEE, 2007.

Frome, A., Huber, D., Kolluri, R., Bülow, T., Malik, J., Recognizing objects in range data using regional point descriptors, Computer Vision-ECCV 2004. Springer Berlin Heidelberg, 2004, pp. 224-237.

Heider, P., Pierre-Pierre, A., Li, R., and Grimm, C., Local shape descriptors, a survey and evaluation, In Proceedings of the 4th Eurographics conference on 3D Object Retrieval, pp. 49-56. Eurographics Association, 2011.

Horn, B., Extended Gaussian images, Proc. of the IEEE, 72(12):1671-1686, December 1984.

Jayanti, S., Yagnanarayanan, K., Iyer, N., and Ramani, K., Developing an engineering shape benchmark for CAD models, Computer-Aided Design, 38, 2006, pp. 939-953.

Johnson, A. E., and Hebert. M., Using spin images for efficient object recognition in cluttered 3D scenes, Pattern Analysis and Machine Intelligence, IEEE Transactions on 21.5, 1999, pp. 433-449.

Kalyanaraman, Y., Boutin, M., and Ramani, K., Effectiveness of convex hull histograms for shape matching, PRECISE technical report, PRE-TR-2005-2, West Lafayette (IN): Purdue University; 2005.

Kazhdan, M, Funkhouser, T., and Rusinkiewicz, S., Rotation invariant spherical harmonic representation of 3D shape descriptors. In Symposium on Geometry Processing, June 2003.

Koenderink, J. J., and van Doorn, A., J., Surface shape and curvature scales, Image and vision computing 10.8, 1992, pp. 557564.

Laga, H., Hiroki, T., and Nakajima, M., Spherical wavelet descriptors for content-based 3D model retrieval, Shape Modeling and Applications, 2006. SMI 2006. IEEE International Conference on. IEEE, 2006.

Lazebnik, S., Schmid, C. and Ponce, J., A sparse texture representation using local affine regions, Pattern Analysis and Machine Intelligence, IEEE Transactions on 27.8, 2005, pp. 1265-1278. 
Leymarie, F. F., and Kimia, B. B., The shock scaffold for representing 3d shape, Visual Form 2001, Springer Berlin Heidelberg, 2001, pp. 216-227.

Li, C., and Hamza, A. B., Symmetry discovery and retrieval of nonrigid 3D shapes using geodesic skeleton paths, Multimedia Tools and Applications, 2013, pp. 1-21.

Lo, T.R and Siebert, J.P., Local feature extraction and matching on range images: 2.5D SIFT, Computer Vision and Image Understanding, 113(12), 2009, pp. 1235-1250.

Lowe, D.G., Distinctive image features from scale-invariant keypoints, International Journal of Computer Vision, 60(2), 2004, pp. 91-110.

MacEachren, A. M., Compactness of geographic shape: Comparison and evaluation of measures, Geografiska Annaler. Series B. Human Geography, 1985, pp. 53-67.

Malik, J., Belongie, S., Leung, T., and Shi, J., Contour and texture analysis for image segmentation. International journal of computer vision, 43(1), 2001, pp. 7-27.

Marton, Z-C., Pangercic, D., Rusu, R. B., Holzbach, A., Beetz, M., Hierarchical object geometric categorization and appearance classification for mobile manipulation, Humanoid Robots (Humanoids), 2010 10th IEEE-RAS International Conference on. IEEE, 2010.

Matas, J., Chum, O., Urban, M., and Pajdla, T., Robust wide-baseline stereo from maximally stable extremal regions, Image and vision computing, 22.10, 2004, pp. 761-767.

Mikolajczyk, K. and Schmid. C., A performance evaluation of local descriptors, Pattern Analysis and Machine Intelligence, IEEE Transactions on, 27.10, 2005, pp. 1615-1630.

Mikolajczyk, K., Tuytelaars, T., Schmid, C, Zisserman, A, Matas, J., Schaffalitzky, F.,Kadir, T. and Gool, L.Van, A Comparison of Affine Region Detectors, International Journal of Computer Vision, 65(1-2), 2005, pp. 43-72.

Mollison, D., Conjecture on the Spread of Infection in Two Dimensions Disproved, Nature, vol. 240, Dec. 1972, pp. 467-468.

Mora, C. F., and Kwan, A. K. H., "Sphericity, shape factor, and convexity measurement of coarse aggregate for concrete using digital image processing." Cement and concrete research 30, no. 3, 2000, pp. 351-358.

Novotni, M., and Klein, R., 3D Zernike descriptors for content based shape retrieval, Proceedings of the eighth ACM symposium on Solid modeling and applications, ACM, 2003.

Ohbuchi, R., Osada, K., Furuya, T., and Banno, T., Salient local visual features for shape-based 3D model retrieval, In Shape Modeling and Applications, 2008. SMI 2008. IEEE International Conference on, IEEE, 2008, pp. 93-102

Osada, R., Funkhouser, T., Chazelle, B. and Dobkin, D., Matching 3D models with shape distributions, Shape Modeling International, ,May, 2001, pp. 154-166.

Papadakis, P., Pratikakis, I., Theoharis, T., Perantonis, S., PANORAMA: a 3D shape descriptor based on panoramic views for unsupervised 3D object retrieval, International Journal of Computer Vision 89.2-3, 2010, pp. 177-192.

Petitjean, M., Chirality and symmetry measures: A trans disciplinary review, Entropy, 5:271-312, 2003. 1

Petros, D., and Axenopoulos, A., A compact multi-view descriptor for 3D object retrieval, Content-Based Multimedia Indexing, 2009. CBMI'09. Seventh International Workshop on. IEEE, 2009.

Rahtu, E., Salo, M., and Heikkila, J., A new convexity measure based on a probabilistic interpretation of images, IEEE PAMI, 28(9):1501-1512, 2006. 1, 2

Rea, H. J., Sung, R., Corney, J. R., Clark, D. E.R., and Taylor, N.K., Interpreting three-dimensional shape distributions, IMechE Journal of Mechanical Engineering Science - Part C 2005;219(C6):553-66.

Rosin, P. L., Measuring shape: ellipticity, rectangularity, and triangularity. Machine Vision and Applications, 14(3):172- 184, 2003.

Rosin, P. L. and Mumford, C. L., A symmetric convexity measure. Computer Vision and Image Understanding, 103(2):101-111, 2006. 2

Rustamov, R. M., Laplace-Beltrami eigenfunctions for deformation invariant shape representation, Proceedings of the fifth Eurographics symposium on Geometry processing, Eurographics Association, 2007.

Rusu, R. B., Bradski, G., Thibaux, R., Hsu, J., Fast 3d recognition and pose using the viewpoint feature histogram, Intelligent Robots and Systems (IROS), 2010 IEEE/RSJ International Conference on. IEEE, 2010.

Rusu, R. B., Blodow, N., and Beetz, M., Fast point feature histograms (fpfh) for 3d registration, Robotics and Automation, ICRA'09. IEEE International Conference on. IEEE, 2009.

Rusu, R. B., and Cousins, S., 3d is here: Point cloud library (pcl), Robotics and Automation (ICRA), 2011 IEEE International Conference on. IEEE, 2011.

Rusu, R. B., Holzbach, A., Beetz, M., and Bradski, G., Detecting and segmenting objects for mobile manipulation, Computer Vision Workshops (ICCV Workshops), 2009 IEEE 12th International Conference on. IEEE, 2009.

Sarfraz, M. S., and Hellwich. O., Head Pose Estimation in Face Recognition Across Pose Scenarios, VISAPP (1), 2008. 
Saupe, D. and Vranic, D. V., 3D model retrieval with spherical harmonics and moments, In B. Radig and S. Florczyk, editors, DAGM 2001, September, 2001, pp. 392-397

Shechtman, E. and Irani, M., Matching local self-similarities across images and videos, Computer Vision and Pattern Recognition, 2007. CVPR'07. IEEE Conference on. IEEE, 2007.

Shilane, P., Min, P., Kazhdan, M., and Funkouser, T., The Princeton shape benchmark, Shape Modeling International, June, 2004.

Steder, B., Rusu, R. B., Konolige, K., Burgard, W., NARF: 3D range image features for object recognition." Workshop on Defining and Solving Realistic Perception Problems in Personal Robotics at the IEEE/RSJ Int. Conf. on Intelligent Robots and Systems (IROS). Vol. 44, 2010.

Stein, F., Medioni, G., Structural indexing: Efficient 3- d object recognition, IEEE Transactions on Pattern Analysis and Machine Intelligence, 14(2), 1992, pp. 125-145

Tombari, F., Salti, S., and Di Stefano, L., Unique signatures of histograms for local surface description, Computer Vision-ECCV 2010. Springer Berlin Heidelberg, 2010, pp. 356-369.

Vranic, D. V., An improvement of rotation invariant 3D shape descriptor based on functions on concentric spheres. In IEEE International Conference on Image Processing (ICIP 2003), volume 3, September 2003, p. 757-760.

Wadell, Hakon. "Volume, shape, and roundness of rock particles." The Journal of Geology, 1932, pp. 443-451.

Zhong, Y., Intrinsic shape signatures: A shape descriptor for 3d object recognition, Computer Vision Workshops (ICCV Workshops), 2009 IEEE 12th International Conference on. IEEE, 2009.

Zhouhui, L., Godil, A., Rosin, P. L., and Sun, X., A new convexity measurement for 3D meshes, In Computer Vision and Pattern Recognition (CVPR), 2012 IEEE Conference on, IEEE, 2012, pp. 119-126.

Zivkovic, Z. and Krose, B., An EM-like algorithm for color-histogram-based object tracking, Computer Vision and Pattern Recognition 2004, CVPR 2004, Proceedings of the 2004 IEEE Computer Society Conference on. Vol. 1. IEEE, 2004.

Žunić, J., Hirota, K., and Rosin, P. L., A Hu moment invariant as a shape circularity measure, Pattern Recognition, 43(1):47-57, 2010, 1

Žunić, J. and Rosin, P. L., Rectilinearity measurements for 'polygons. IEEE PAMI, 25(9):1193-1200, 2003.1

Žunić, J. and Rosin, P. L., A new convexity measure for polygons. IEEE PAMI, 26(7):923-934, 2004. 1, 2,3 